JOURNAL OF THE

AMERICAN MATHEMATICAL SOCIETY

Volume 15, Number 2, Pages 367-417

S 0894-0347(01)00388-5

Article electronically published on December 31, 2001

\title{
ON THE GEOMETRIC LANGLANDS CONJECTURE
}

\author{
E. FRENKEL, D. GAITSGORY, AND K. VILONEN
}

\section{INTRODUCTION}

0.1. Background. Let $X$ be a smooth, complete, geometrically connected curve over the finite field $\mathbb{F}_{q}$. Denote by $F$ the field of rational functions on $X$ and by $\mathbb{A}$ the ring of adèles of $F$. The Langlands conjecture, recently proved by L. Lafforgue Laf], establishes a correspondence between cuspidal automorphic forms on the group $G L_{n}(\mathbb{A})$ and irreducible, almost everywhere unramified, $n$-dimensional $\ell-$ adic representations of the Galois group of $\bar{F}$ over $F$ (more precisely, of the Weil group).

An unramified automorphic form on the group $G L_{n}(\mathbb{A})$ can be viewed as a function on the set $\operatorname{Bun}_{n}\left(\mathbb{F}_{q}\right)$ of isomorphism classes of rank $n$ bundles on the curve $X$. The set $\operatorname{Bun}_{n}\left(\mathbb{F}_{q}\right)$ is the set of $\mathbb{F}_{q}$-points of $\operatorname{Bun}_{n}$, the algebraic stack of rank $n$ bundles on $X$. According to Grothendieck's "faisceaux-fonctions" correspondence, one can attach to an $\ell$-adic perverse sheaf on $\operatorname{Bun}_{n}$ a function on $\operatorname{Bun}_{n}\left(\mathbb{F}_{q}\right)$ by taking the traces of the Frobenius on the stalks. V. Drinfeld's geometric proof Dr] of the Langlands conjecture for $G L_{2}$ (and earlier geometric interpretation of the abelian class field theory by P. Deligne, see [Lau1]) opened the possibility that automorphic forms may be constructed as the functions associated to perverse sheaves on $\operatorname{Bun}_{n}$.

Thus, one is led to a geometric version of the Langlands conjecture proposed by V. Drinfeld and G. Laumon: for each geometrically irreducible rank $n$ local system $E$ on $X$ there exists a perverse sheaf Aut $_{E}$ on $\operatorname{Bun}_{n}$ (irreducible on each component), which is a Hecke eigensheaf with respect to $E$, in an appropriate sense (see [Lau1] or Sect. 1 below for the precise formulation). Moreover, the geometric Langlands conjecture can be made over an arbitrary field $\mathbb{k}$.

Building on the ideas of Drinfeld's work $\mathrm{Dr}$, G. Laumon gave a conjectural construction of $\mathrm{Aut}_{E}$ in Lau1, Lau2. More precisely, he attached to each rank $n$ local system $E$ on $X$ a complex of perverse sheaves Aut ${ }_{E}^{\prime}$ on the moduli stack Bun ${ }_{n}^{\prime}$ of pairs $\{\mathcal{M}, s\}$, where $\mathcal{M} \in \operatorname{Bun}_{n}$ is a rank $n$ bundle on $X$ and $s$ is a regular nonzero section of $\mathcal{M}$. He conjectured that if $E$ is geometrically irreducible, then this sheaf descends to a perverse sheaf $\mathrm{Aut}_{E}$ on $\mathrm{Bun}_{n}$ (irreducible on each component), which is a Hecke eigensheaf with respect to $E$.

In our previous work [FGKV], joint with D. Kazhdan, we have shown that the function on $\operatorname{Bun}_{n}^{\prime}\left(\mathbb{F}_{q}\right)$ associated to $\operatorname{Aut}_{E}^{\prime}$ agrees with the function constructed previously by I.I. Piatetskii-Shapiro [PS1] and J.A. Shalika [Sha], as anticipated by Laumon Lau2. This provided a consistency check for Laumon's construction.

Received by the editors February 14, 2001.

2000 Mathematics Subject Classification. Primary 11R39, 11F70; Secondary 14H60, 22E55. 
In this paper we formulate a certain vanishing conjecture, and prove that Laumon's construction indeed produces a perverse sheaf $\mathrm{Aut}_{E}$ on $\mathrm{Bun}_{n}$ with desired properties, when the vanishing conjecture holds. In other words, the vanishing conjecture implies the geometric Langlands conjecture, over any field $\mathbb{k}$. For the sake of definiteness, we work in this paper with a field $\mathbb{k}$ of characteristic $p>0$, but our results (with appropriate modifications, such as switching from perverse sheaves to $\mathcal{D}$-modules) remain valid if $\operatorname{char} \mathbb{k}=0$.

Moreover, in the case when $\mathbb{k}$ is a finite field, we derive the vanishing conjecture (and hence the geometric Langlands conjecture) from the results of L. Lafforgue Laf].

To give the reader a feel for the vanishing conjecture, we give here one of its formulations (see Sect. 2 for more details). Let $\mathcal{M}$ and $\mathcal{M}{ }^{\prime}$ be two vector bundles on $X$ of rank $k$, such that $\operatorname{deg}\left(\mathcal{M}^{\prime}\right)-\operatorname{deg}(\mathcal{M})=d>0$. Consider the space $\operatorname{Hom}^{0}\left(\mathcal{M}, \mathcal{M}^{\prime}\right)$ of injective sheaf homomorphisms $\mathcal{M} \hookrightarrow \mathcal{M}^{\prime}$. Let $\operatorname{Coh}_{0}^{d}$ be the algebraic stack which classifies torsion sheaves of length $d$, and let $\pi: \operatorname{Hom}^{0}\left(\mathcal{N}, \mathcal{N}^{\prime}\right) \rightarrow \operatorname{Coh}_{0}^{d}$ be the natural morphism sending $\mathcal{M} \hookrightarrow \mathcal{M}^{\prime}$ to $\mathcal{M}^{\prime} / \mathcal{M}$.

G. Laumon [Lau1] has defined a remarkable perverse sheaf $\mathcal{L}_{E}^{d}$ on $\operatorname{Coh}_{0}^{d}$ for any local system $E$ of rank $n$ on $X$. The vanishing conjecture states that if $E$ is irreducible and $n>k$, then

$$
H^{\bullet}\left(\operatorname{Hom}^{0}\left(\mathcal{M}, \mathcal{M}^{\prime}\right), \pi^{*}\left(\mathcal{L}_{E}^{d}\right)\right)=0, \quad \forall d>k n(2 g-2)
$$

0.2. Contents. The paper is organized as follows:

In Sect. 1 we define Hecke functors and state the geometric Langlands conjecture. We want to draw the reader's attention to the fact that our formulation is different from that given in [Lau1] in two respects. The Hecke property is defined here using only the first Hecke functor; according to Proposition 1.5, this implies the Hecke property with respect to the other Hecke functors. We also do not require the cuspidality property in the statement of the conjecture, because we show in Sect. 9 that the cuspidality of a Hecke eigensheaf follows from the vanishing conjecture.

In Sect. 2 we recall the definition of Laumon's sheaf and state our vanishing conjecture.

In Sect. 3 we present two constructions of Aut $_{E}$ following Laumon [Lau1, Lau2] (see also [FGKV]). A third construction, which uses the Whittaker sheaves is given in Sect. 4. This construction is the exact geometric analogue of the construction of Piatetskii-Shapiro [PS1] and Shalika Sha] at the level of functions. The reader is referred to Sect. 3.10 for a summary of the relationship between the three constructions and the strategy of our proof.

In Sects. 5-9 we derive the geometric Langlands conjecture assuming that the vanishing conjecture is true. Sect. 5 is devoted to the proof of the cleanness property in Laumon's construction. In Sect. 6 we prove that the sheaf Aut $_{E}^{\prime}$ on $\mathrm{Bun}_{n}^{\prime}$ descends to a perverse sheaf $\mathrm{Aut}_{E}$ on $\mathrm{Bun}_{n}$. In Sects. 7 and 8 we give two alternative proofs of the Hecke property of Aut $_{E}$. We then show in Sect. 9 that the perverse sheaf $\mathrm{Aut}_{E}$ is cuspidal.

In Sect. 10 we derive the vanishing conjecture from results of L. Lafforgue Laf] when $\mathbb{k}$ is a finite field.

The Appendix contains proofs of some results concerning the Whittaker sheaves, which are not necessary for our proof, but are conceptually important. 
0.3. Notation and conventions. Throughout this paper, $\mathbb{k}$ will be a ground field of characteristic $p>0$ and $X$ will be a smooth complete geometrically connected curve over $\mathbb{k}$ of genus $g>1$.

This paper deals with $\overline{\mathbb{Q}}_{\ell}$-adic perverse sheaves and complexes of perverse sheaves on various schemes over $\mathbb{k}$, where $\ell$ is a prime with $(\ell, p)=1$. In particular, by a local system on $X$ we will understand a smooth $\ell$-adic sheaf over $X$. For brevity, we will refer to a geometrically irreducible local system simply as an irreducible local system.

When $\mathbb{k}=\mathbb{F}_{q}$ we work with Weil sheaves (see $[\mathrm{De}]$ ), instead of sheaves defined over $\mathbb{F}_{q}$. We choose a square root of $q$ in $\overline{\mathbb{Q}}_{\ell}$, which defines a half-integral Tate twist $\overline{\mathbb{Q}}_{\ell}\left(\frac{1}{2}\right)$.

In addition to $\mathbb{k}$-schemes, we will extensively use algebraic stacks in the smooth topology (over $\mathbb{k}$ ); see $\mathrm{LMB}$. If $G$ is an algebraic group, we define $\mathrm{Bun}_{G}$ as a stack that classifies $G$-bundles on $X$. This means that $\operatorname{Hom}\left(S, \operatorname{Bun}_{G}\right)$ is the groupoid whose objects are $H$-bundles on $X \times S$ and morphisms are isomorphisms of these bundles. The pull-back functor for a morphism $S_{1} \rightarrow S_{2}$ is defined in a natural way.

When $G=G L_{n}, \operatorname{Bun}_{G}$ coincides with Bun $n$, the moduli stack of rank $n$ vector bundles on $X$. We write $\operatorname{Bun}_{n}^{d}$ for the connected component of $\operatorname{Bun}_{n}$ corresponding to rank $n$ vector bundles of degree $d$.

For an algebraic stack $y$ we will use the notation $\mathrm{D}(\mathrm{y})$ for the derived category of $\overline{\mathbb{Q}}_{\ell}$-adic perverse sheaves on $y$. We refer the reader to Sect. 1.4 of [FGV] for our conventions regarding this category. When we discuss objects of the derived category, the cohomological grading should always be understood in the perverse sense. In addition, for a morphism $f: y_{1} \rightarrow y_{2}$, the functors $f_{!}, f_{*}, f^{*}$ and $f^{!}$ should be understood "in the derived sense".

If $y$ is a stack over $\mathbb{k}=\mathbb{F}_{q}$ and $\mathbb{F}_{q_{1}}$ is an extension of $\mathbb{F}_{q}$, we denote by $y\left(\mathbb{F}_{q_{1}}\right)$ the set of isomorphism classes of objects of the groupoid $\operatorname{Hom}\left(\operatorname{Spec} \mathbb{F}_{q_{1}}, y\right)$. If $\mathcal{S}$ is a perverse sheaf or a complex of perverse sheaves on $y$, then $y\left(\mathbb{F}_{q_{1}}\right)$ is endowed with the function "alternating sum of traces of the Frobenius on stalks" (as in [De]). We denote this function by $\mathbf{f}_{q_{1}}(\mathcal{S})$.

For the general definitions related to the Langlands correspondence and the formulation of the Langlands conjecture we refer the reader to [Lau1, Sect. 1, and FGKV], Sect. 2. In particular, the notions of cuspidal automorphic function or Hecke eigenfunction on $G L_{n}(\mathbb{A})$ may be found there.

\section{Hecke eigensheaves}

In this section we introduce the Hecke functors and state the geometric Langlands conjecture.

1.1. Hecke functors. Consider the following correspondence:

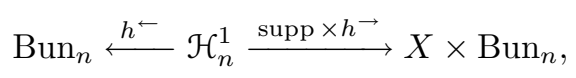

where the stack $\mathcal{H}_{n}^{1}$ classifies quadruples $\left(x, \mathcal{M}, \mathcal{M}^{\prime}, \beta: \mathcal{M}^{\prime} \hookrightarrow \mathcal{M}\right)$, with $x \in X$, $\mathcal{M}^{\prime}, \mathcal{M} \in \operatorname{Bun}_{n}$, such that $\mathcal{M} / \mathcal{M}^{\prime}$ is the simple skyscraper sheaf supported at $x$, i.e., $\mathcal{M} / \mathcal{M}^{\prime}$ is (non-canonically) isomorphic to $\mathcal{O}_{X}(x) / \mathcal{O}_{X}$. The morphisms $h^{\leftarrow}, h^{\rightarrow}$, and supp are given by $h^{\leftarrow}\left(x, \mathcal{M}, \mathcal{M}^{\prime}\right)=\mathcal{M}, h^{\rightarrow}\left(x, \mathcal{M}, \mathcal{M}^{\prime}\right)=\mathcal{M}^{\prime}$, and $\operatorname{supp}\left(x, \mathcal{M}, \mathcal{M}^{\prime}\right)=x$. 
The Hecke functor $\mathrm{H}_{n}^{1}: \mathrm{D}\left(\mathrm{Bun}_{n}\right) \rightarrow \mathrm{D}\left(X \times \mathrm{Bun}_{n}\right)$ is defined by the formula

$$
\mathrm{H}_{n}^{1}(\mathcal{K})=\left(\operatorname{supp} \times h^{\rightarrow}\right) ! h^{\leftarrow *}(\mathcal{K}) \otimes \overline{\mathbb{Q}}_{\ell}\left(\frac{n-1}{2}\right)[n-1] .
$$

Consider the $i$-th iteration of $\mathrm{H}_{n}^{1}$ :

$$
\left(\mathrm{H}_{n}^{1}\right)^{\bigotimes i}: \mathrm{D}\left(\mathrm{Bun}_{n}\right) \rightarrow \mathrm{D}\left(X^{i} \times \operatorname{Bun}_{n}\right) .
$$

Let $\Delta$ denote the divisor in $X^{i}$ consisting of the pairwise diagonals. Note that for any $\mathcal{K} \in \mathrm{D}\left(\operatorname{Bun}_{n}\right)$ the restriction $\left.\left(H_{n}^{1}\right)^{\bigotimes i}(\mathcal{K})\right|_{\left(X^{i}-\Delta\right) \times \mathrm{Bun}_{n}}$ is naturally equivariant with respect to the action of the symmetric group $S_{i}$ on $X^{i}-\Delta$.

Consider a rank $n$ local system $E$ on $X$. We say that $\mathcal{K} \in \mathrm{D}\left(\mathrm{Bun}_{n}\right)$ is a Hecke eigensheaf, or that it has a Hecke property with respect to $E$, if $\mathcal{K} \neq 0$ and there exists an isomorphism

$$
\mathrm{H}_{n}^{1}(\mathcal{K}) \simeq E \otimes \mathcal{K}
$$

such that the resulting map

$$
\left.\left.\left(\mathrm{H}_{n}^{1}\right)^{\bigotimes 2}(\mathcal{K})\right|_{(X \times X-\Delta) \times \mathrm{Bun}_{n}} \rightarrow E \otimes E \otimes \mathcal{K}\right|_{(X \times X-\Delta) \times \mathrm{Bun}_{n}}
$$

is $S_{2}$-equivariant. This i mplies that

$$
\left.\left.\left(\mathrm{H}_{n}^{1}\right)^{\bigotimes i}(\mathcal{K})\right|_{\left(X^{i}-\Delta\right) \times \mathrm{Bun}_{n}} \rightarrow E^{\bigotimes i} \otimes \mathcal{K}\right|_{\left(X^{i}-\Delta\right) \times \mathrm{Bun}_{n}}
$$

is $S_{i}$-equivariant for any $i$.

1.2. Statement of the geometric Langlands conjecture. We are now ready to formulate the unramified geometric Langlands conjecture for $G L_{n}$ :

1.3. Conjecture. For each irreducible rank $n$ local system $E$ on $X$ there exists a perverse sheaf $\mathrm{Aut}_{E}$ on $\mathrm{Bun}_{n}$, irreducible on each connected component $\mathrm{Bun}_{n}^{d}$, which is a Hecke eigensheaf with respect to $E$. Ga]).

Conjecture 1.3 has been proved by Drinfeld $[\mathrm{Dr}]$ in the case when $n=2$ (see also

In this paper we reduce Conjecture 1.3 to the Vanishing Conjecture 2.3. Then, in Sect. 10 we will show that when $\mathbb{k}$ is a finite field $\mathbb{F}_{q}$, the Vanishing Conjecture follows from recent results of Lafforgue [Laf].

1.4. Other Hecke functors. In addition to the functor $\mathrm{H}_{n}^{1}$, we also have Hecke functors $\mathrm{H}_{n}^{i}: \mathrm{D}\left(\operatorname{Bun}_{n}\right) \rightarrow \mathrm{D}\left(X \times \operatorname{Bun}_{n}\right)$ for $i=2, \ldots, n$. To define them, consider the stack $\mathcal{H}_{n}^{i}$ which classifies quadruples

$$
\left(x, \mathcal{N}, \mathcal{M}^{\prime}, \beta: \mathcal{M}^{\prime} \hookrightarrow \mathcal{M}\right)
$$

where $x \in X, \mathcal{M}^{\prime}, \mathcal{M} \in \operatorname{Bun}_{n}$ such that $\mathcal{M}^{\prime} \subset \mathcal{M} \subset \mathcal{M}^{\prime}(x)$, and length $\left(\mathcal{M} / \mathcal{M}^{\prime}\right)=i$.

As in the case of $\mathcal{H}_{n}^{1}$, we have a diagram

$$
\operatorname{Bun}_{n} \stackrel{h^{\leftarrow}}{\longleftarrow} \mathcal{H}_{n}^{i} \stackrel{\operatorname{supp} \times h^{\rightarrow}}{\longrightarrow} X \times \operatorname{Bun}_{n},
$$

and the functor $\mathrm{H}_{n}^{i}$ is defined by the formula

$$
\mathrm{H}_{n}^{i}(\mathcal{K})=\left(\operatorname{supp} \times h^{\rightarrow}\right) ! h^{\leftarrow *}(\mathcal{K}) \otimes \overline{\mathbb{Q}}_{\ell}\left(\frac{(n-i) i}{2}\right)[(n-i) i] .
$$

The following result is borrowed from [Ga]:

1.5. Proposition. Let $\mathcal{K}$ be a Hecke eigensheaf with respect to $E$. Then for $i=$ $1, \ldots, n$ we have isomorphisms $\mathrm{H}_{n}^{i}(\mathcal{K}) \simeq \Lambda^{i} E \otimes \mathcal{K}$. 
Proof. Consider the stack $\operatorname{Mod}_{n}^{-i}$ of "lower modifications of length $i$ ", which classifies the data of triples $\left(\mathcal{M}, \mathcal{M}^{\prime}, \beta: \mathcal{M}^{\prime} \hookrightarrow \mathcal{M}\right)$, where $\mathcal{M}, \mathcal{M}^{\prime} \in \operatorname{Bun}_{n}$ and $\beta$ is an embedding of coherent sheaves such that the quotient $\mathcal{M} / \mathcal{M}^{\prime}$ is a torsion sheaf of length $i$.

Let $X^{(i)}$ be the $i$-th symmetric power of $X$. We have a natural morphism supp : $\operatorname{Mod}_{n}^{-i} \rightarrow X^{(i)}$, which associates to $\left(\mathcal{M}^{\prime}, \mathcal{M}, \beta\right)$ as above the divisor of zeros of the induced map $\operatorname{det} \mathcal{M}^{\prime} \rightarrow \operatorname{det} \mathcal{M}$.

Denote by $\mathcal{H}_{n}^{i,+}$ the preimage in $\operatorname{Mod}_{n}^{-i}$ of the main diagonal $X \subset X^{(i)}$. Note that $\mathcal{H}_{n}^{i}$ is naturally a closed substack in $\mathcal{H}_{n}^{i,+}$.

Consider the stack $\widetilde{\operatorname{Mod}}_{n}^{-i}$, which classifies the data $\left(\mathcal{M}^{\prime}=\mathcal{M}_{0} \subset \mathcal{M}_{1} \subset \ldots \subset\right.$ $\left.\mathcal{M}_{i}=\mathcal{M}\right)$, where each $\mathcal{M}_{j}$ is a rank $n$ vector bundle, and $\mathcal{M}_{j} / \mathcal{M}_{j-1}$ is a simple skyscraper sheaf. There is a natural proper map $p: \widetilde{\operatorname{Mod}}_{n}^{-i} \rightarrow \operatorname{Mod}_{n}^{-i}$, which "forgets" the middle terms of the filtration.

There is also a natural map $\widetilde{\operatorname{supp}}:{\widetilde{\operatorname{Mod}_{n}^{-i}}}_{\rightarrow} X^{i}$ such that if sym $: X^{i} \rightarrow X^{(i)}$ denotes the symmetrization map, we have sym $\circ \widetilde{\operatorname{supp}}=\operatorname{supp} \circ p: \widetilde{\operatorname{Mod}}_{n}^{-i} \rightarrow X^{(i)}$.

The open substack $\widetilde{\operatorname{supp}}^{-1}\left(X^{i}-\Delta\right)$ of $\widetilde{\operatorname{Mod}}_{n}^{-i}$ is isomorphic to the fiber product $\operatorname{Mod}_{n}^{-i} \underset{X^{(i)}}{\times}\left(X^{i}-\Delta\right)$.

The map $p$ is known to be small (see, e.g., [Lau1]). This implies that the complex

$$
\mathcal{S} p r:=p_{!}\left(\overline{\mathbb{Q}}_{\ell}\left(\frac{i(n-1)}{2}\right)\right)[i(n-1)]
$$

on $\operatorname{Mod}_{n}^{-i}$ is perverse (up to the cohomological shift by $n^{2} \cdot(g-1)=\operatorname{dim}\left(\operatorname{Bun}_{n}\right)$ ) and is a Goresky-MacPherson extension of its restriction to $\operatorname{supp}^{-1}\left(X^{(i)}-\Delta\right)$. In particular, $\mathcal{S} p r$ carries a canonical $S_{i}$-action and $(\mathcal{S} p r)^{S_{i}} \simeq \overline{\mathbb{Q}}_{\ell}\left(\frac{i(n-1)}{2}\right)[i(n-1)]$.

Let $h^{\leftarrow}$ (resp., $h \rightarrow$ ) denote the morphism $\operatorname{Mod}_{n}^{-i} \rightarrow \operatorname{Bun}_{n}$, which sends a triple $\left(\mathcal{M}, \mathcal{M}^{\prime}, \beta\right)$ to $\mathcal{M}$ (resp., $\left.\mathcal{M}^{\prime}\right)$. By construction, for any $\mathcal{K} \in \mathrm{D}\left(\mathrm{Bun}_{n}\right)$,

$$
\left(\operatorname{supp} \times h^{\rightarrow}\right) !\left(h^{\leftarrow *}(\mathcal{K}) \otimes \mathcal{S} p r\right) \simeq(\operatorname{sym} \times \mathrm{id}) !\left(\mathrm{H}_{n}^{1}\right)^{\bigotimes i}(\mathcal{K}) .
$$

Thus, if $\mathcal{K}$ is a Hecke eigensheaf with respect to $E$, we obtain an $S_{i}$-equivariant isomorphism

$$
\left(\operatorname{supp} \times h^{\rightarrow}\right)_{!}\left(h^{\leftarrow *}(\mathcal{K}) \otimes \mathcal{S} p r\right) \simeq \operatorname{sym}_{!}\left(E^{\bigotimes i}\right) \otimes \mathcal{K} .
$$

To conclude the proof, we pass to the isotypic components of the sign representation of $S_{i}$ on both sides of formula (1.5) and restrict the resulting isotypic components to the main diagonal $X \subset X^{(i)}$. By this process the RHS of (1.5) tautologically yields $\Lambda^{i} E \otimes \mathcal{K}$. Thus, it remains to show that the LHS yields $\mathrm{H}_{n}^{i}(\mathcal{K})$. To see this, it suffices to note that $\left.\operatorname{Hom}_{S_{i}}(\operatorname{sign}, \mathcal{S} p r)\right|_{\mathcal{H}_{n}^{i,+}}$ is isomorphic to the constant sheaf on $\mathcal{H}_{n}^{i}$ tensored by $\overline{\mathbb{Q}}_{\ell}\left(\frac{(n-i) i}{2}\right)[(n-i) i]$, by the Springer theory $[\mathrm{BM}, \mathrm{Sp}]$.

The isomorphisms constructed in the above proposition have an additional property. To state it, let $\sigma$ be the transposition acting on $X \times X$ and let $i, j \in\{1, \ldots, n\}$. Clearly, the functors

$$
\left.\mathcal{K} \mapsto\left(\mathrm{H}_{n}^{i} \times \mathrm{id}\right) \circ \mathrm{H}_{n}^{j}(\mathcal{K})\right|_{(X \times X-\Delta) \times \mathrm{Bun}_{n}}
$$

and

$$
\left.\mathcal{K} \mapsto \sigma^{*} \circ\left(\mathrm{H}_{n}^{j} \times \mathrm{id}\right) \circ \mathrm{H}_{n}^{i}(\mathcal{K})\right|_{(X \times X-\Delta) \times \mathrm{Bun}_{n}}
$$


from $\mathrm{D}\left(\mathrm{Bun}_{n}\right)$ to $\mathrm{D}\left((X \times X-\Delta) \times \mathrm{Bun}_{n}\right)$ are naturally isomorphic. Hence, for a Hecke eigensheaf $\mathcal{K}$, the following diagram is commutative:

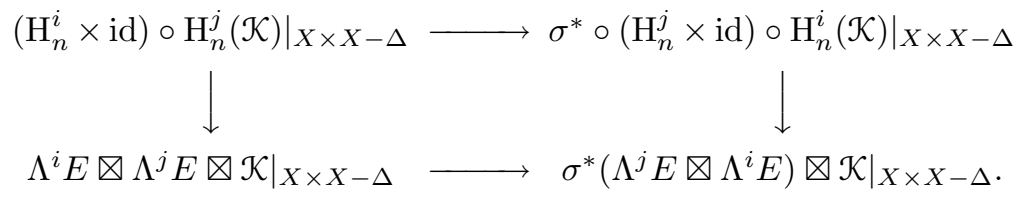

Finally, let us consider the Hecke functor $\mathrm{H}_{n}^{n}$. By definition, this is the pull-back under the morphism mult : $X \times \operatorname{Bun}_{n} \rightarrow \operatorname{Bun}_{n}$ given by $(x, \mathcal{N}) \mapsto \mathcal{M}(x)$. Hence if $\mathcal{K}$ is a Hecke eigensheaf with respect to $E$, then

$$
\operatorname{mult}^{*}(\mathcal{K}) \simeq \Lambda^{n} E \otimes \mathcal{K} .
$$

\section{The VAnishing CONJeCture}

Denote by $\mathrm{Coh}_{n}$ the stack classifying coherent sheaves on $X$ of generic rank $n$. More precisely, for each $\mathbb{k}$-scheme $S, \operatorname{Hom}\left(S, \operatorname{Coh}_{n}\right)$ is the groupoid, whose objects are coherent sheaves $\mathcal{M}_{S}$ on $X \times S$, which are flat over $S$, and such that over every geometric point $s \in S, \mathcal{M}_{s}$ is generically of rank $n$. We write $\operatorname{Coh}_{n}^{d}$ for the substack corresponding to coherent sheaves of generic rank $n$ and degree $d$.

2.1. Laumon's sheaf. In Lau2 Laumon associated to an arbitrary local system $E$ of rank $n$ on $X$ a perverse sheaf $\mathcal{L}_{E}$ on $C o h_{0}$. Let us recall his construction. Denote by $\operatorname{Coh}_{0}^{\text {rss }}$ the open substack of $\operatorname{Coh}_{0}$ corresponding to regular semisimple torsion sheaves. Thus, a geometric point of Coh $_{0}$ belongs to $\operatorname{Coh}_{0}^{\mathrm{rss}}$ if the corresponding coherent sheaf on $X$ is a direct sum of skyscraper sheaves of length one supported at distinct points of $X$. Let $\operatorname{Coh}_{0}^{\mathrm{rss}, d}=\operatorname{Coh}_{0}^{\mathrm{rss}} \cap \operatorname{Coh}^{d}$. We have a natural smooth $\operatorname{map}\left(X^{(d)}-\Delta\right) \rightarrow \operatorname{Coh}_{0}^{\mathrm{rss}, d}$.

Let $E^{(d)}$ be the $d$-th symmetric power of $E$, i.e., $E^{(d)}=\operatorname{sym}_{1}\left(E^{\bigotimes d}\right)^{S_{d}}$, where sym : $X^{d} \rightarrow X^{(d)}$. This is a perverse sheaf on $X^{(d)}$, and its restriction $\left.E^{(d)}\right|_{X^{(d)}-\Delta}$ is a local system, which descends to a local system $\stackrel{\circ}{\mathcal{L}}_{E}^{d}$ on $\operatorname{Coh}_{0}^{\text {rss, } d}$. The perverse sheaf $\mathcal{L}_{E}^{d}$ on $\mathcal{C} h_{0}^{d}$ is by definition the Goresky-MacPherson extension of $\stackrel{\circ}{\mathcal{L}}_{E}^{d}$ from $\operatorname{Coh}_{0}^{\text {rss, } d}$ to $\operatorname{Coh}_{0}^{d}$. We denote by $\mathcal{L}_{E}$ the perverse sheaf on $\operatorname{Coh}_{0}$, whose restriction to $\operatorname{Coh}_{0}^{d}$ equals $\mathcal{L}_{E}^{d}$.

2.2. The averaging functor. Using the perverse sheaf $\mathcal{L}_{E}^{d}$ we define the averaging functor $\mathrm{H}_{k, E}^{d}: \mathrm{D}\left(\operatorname{Bun}_{k}\right) \rightarrow \mathrm{D}\left(\operatorname{Bun}_{k}\right)$. We stress that the positive integer $k$ is independent of $n$, the rank of the local system $E$.

For $d \geq 0$, introduce the stack $\operatorname{Mod}_{k}^{d}$, which classifies the data of triples $\left(\mathcal{M}, \mathcal{M}^{\prime}\right.$, $\beta: \mathcal{M} \hookrightarrow \mathcal{M}^{\prime}$ ), where $\mathcal{M}, \mathcal{N}^{\prime} \in \operatorname{Bun}_{k}$ and $\beta$ is an embedding of coherent sheaves such that the quotient $\mathcal{M}^{\prime} / \mathcal{M}$ is a torsion sheaf of length $d$, and the diagram

$$
\operatorname{Bun}_{k} \stackrel{h^{\leftarrow}}{\longleftarrow} \operatorname{Mod}_{k}^{d} \stackrel{h \rightarrow}{\longrightarrow} \operatorname{Bun}_{k},
$$

where $h^{\leftarrow}$ (resp., $h^{\rightarrow}$ ) denotes the morphism sending a triple $\left(\mathcal{M}, \mathcal{M}^{\prime}, \beta\right)$ to $\mathcal{M}$ (resp., $\left.\mathcal{M}^{\prime}\right)$. In addition, we have a natural smooth morphism $\pi: \operatorname{Mod}_{k}^{d} \rightarrow \operatorname{Coh}_{0}^{d}$, which sends a triple $\left(\mathcal{M}, \mathcal{M}^{\prime}, \beta\right)$ to the torsion sheaf $\mathcal{M}^{\prime} / \mathcal{M}$.

Note that $\operatorname{Mod}_{n}^{d}$ is isomorphic to the stack $\operatorname{Mod}_{n}^{-d}$ which was used in the proof of Proposition 1.5. Under this isomorphism the maps $h^{\rightarrow}$ and $h^{\leftarrow}$ are reversed. 
The averaging functor $\mathrm{H}_{k, E}^{d}: \mathrm{D}\left(\mathrm{Bun}_{k}\right) \rightarrow \mathrm{D}\left(\operatorname{Bun}_{k}\right)$ is defined by the formula

$$
\mathcal{K} \mapsto h_{!}\left(h^{\leftarrow *}(\mathcal{K}) \otimes \pi^{*}\left(\mathcal{L}_{E}^{d}\right)\right) \otimes \overline{\mathbb{Q}}_{\ell}\left(\frac{d \cdot k}{2}\right)[d \cdot k] .
$$

2.3. Vanishing Conjecture. Assume that $E$ is an irreducible local system of rank $n$. Then for all $k=1, \ldots, n-1$ and all d satisfying $d>k n(2 g-2)$, the functor $\mathrm{H}_{k, E}^{d}$ is identically equal to 0 .

The statement of the Vanishing Conjecture is known to be true for $k=1$ (see below).

The goal of this paper is to show that if Conjecture 2.3 holds for any given irreducible rank $n$ local system $E$, then the geometric Langlands Conjecture 1.3 holds for $E$. In addition, in Sect. 10 we will prove Conjecture 2.3 in the case when $\mathbb{k}$ is a finite field $\mathbb{F}_{q}$ if the following statements are true (see $\mathrm{BBD}$ for the definition of a pure local system):

(a) $E$ is pure (up to a twist by a one-dimensional representation of the Weil group of $\mathbb{F}_{q}$ ),

and either

(b) there exists a cuspidal Hecke eigenfunction associated to the pull-back of $E$

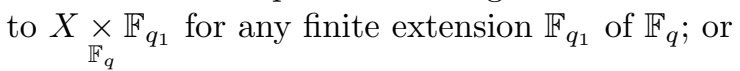

$\left(\mathrm{b}^{\prime}\right)$ the space of unramified cuspidal automorphic functions on the group $G L_{k}$ over the adèles is spanned by the Hecke eigenfunctions associated to rank $k$

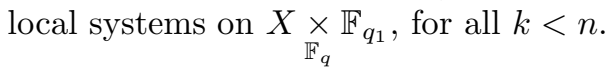

The statements (a),(b), (b') follow from the recent work of Lafforgue [Laf] (note that (b) and ( $\left.\mathrm{b}^{\prime}\right)$ are specified by the Langlands conjecture at the level of functions). Therefore, Lafforgue's results together with the results of the present paper imply Conjecture 2.3 and hence Conjecture 1.3 over a finite field $\mathbb{k}$.

2.4. A reformulation of the Vanishing Conjecture. Let $\mathcal{M}$ and $\mathcal{M}^{\prime}$ be two rank $k$ vector bundles on $X$ and let us write $\operatorname{Hom}^{0}\left(\mathcal{M}, \mathcal{M}^{\prime}\right)$ for the open subset of injective maps in the vector space $\operatorname{Hom}\left(\mathcal{M}, \mathcal{M}^{\prime}\right)$. There is a natural morphism $\pi: \operatorname{Hom}^{0}\left(\mathcal{M}^{\prime}, \mathcal{M}\right) \rightarrow \operatorname{Coh}_{0}^{d}$, where $d=\operatorname{deg}\left(\mathcal{M}^{\prime}\right)-\operatorname{deg}(\mathcal{M})$, which maps $\left(\mathcal{M} \hookrightarrow \mathcal{M}^{\prime}\right)$ to $\mathcal{M}^{\prime} / \mathcal{M}$.

2.5. Conjecture. Under the assumptions on $E$ and $d$ given in Conjecture 2.3

$$
H^{\bullet}\left(\operatorname{Hom}^{0}\left(\mathcal{M}, \mathcal{M}^{\prime}\right), \pi^{*}\left(\mathcal{L}_{E}^{d}\right)\right)=0 .
$$

Conjectures 2.3 and 2.5 are equivalent. Indeed, consider the complex

$$
\left(h^{\leftarrow} \times h^{\rightarrow}\right) ! \pi^{*}\left(\mathcal{L}_{E}^{d}\right) \in \mathrm{D}\left(\operatorname{Bun}_{k} \times \operatorname{Bun}_{k}\right) .
$$

Conjecture 2.3 is equivalent to the statement that this complex equals 0. But its stalk at $\left(\mathcal{M}, \mathcal{M}^{\prime}\right) \in \operatorname{Bun}_{k} \times \operatorname{Bun}_{k}$ is precisely the cohomology

$$
H^{\bullet}\left(\operatorname{Hom}^{0}\left(\mathcal{M}^{\prime}, \mathcal{M}\right), \pi^{*}\left(\mathcal{L}_{E}^{d}\right)\right) .
$$

2.6. Proof of the statement of Conjecture 2.3 in the case $k=1$. Recall the Deligne vanishing theorem (see the Appendix of $[\mathrm{Dr}]$ ):

Let $\mathrm{AJ}: X^{(d)} \rightarrow \operatorname{Pic}^{d}(X)$ be the Abel-Jacobi map, and let $E$ be an irreducible local system of rank $n>1$. Then for $d>n(2 g-2)$

$$
(\mathrm{AJ}) !\left(E^{(d)}\right)=0 \text {. }
$$


This theorem implies the case $k=1$ of Conjecture 2.3. Indeed, consider the morphism $X^{(d)} \rightarrow \operatorname{Coh}_{0}^{d}$ that associates to a divisor $D$ the torsion sheaf $\mathcal{O}_{X}(D) / \mathcal{O}_{X}$. This morphism is smooth and its image is the open substack $\operatorname{Coh}_{0}^{\mathrm{r}, d}$ of $\operatorname{Coh}_{0}^{d}$ corresponding to those torsion sheaves $\mathcal{T}$ on $X$ for which $\operatorname{dim}_{\mathbb{k}}(\operatorname{End}(\mathcal{T}))=\operatorname{length}(\mathcal{T})$ (such torsion sheaves are called regular). Clearly, $\operatorname{Coh}_{0}^{\mathrm{rss}, d} \subset \operatorname{Coh}_{0}^{\mathrm{r}, d}$. Since the Laumon sheaf $\mathcal{L}_{E}^{d}$ is an irreducible perverse sheaf on $\mathcal{C} o h_{0}^{d}$, and $E^{(d)}$ is an irreducible perverse sheaf on $X^{(d)}$, we obtain that the pull-back of $\mathcal{L}_{E}^{d}$ under the morphism $X^{(d)} \rightarrow \operatorname{Coh}_{0}^{d}$ is isomorphic to $E^{(d)}$. Observe now that the diagram of stacks

$$
\operatorname{Bun}_{1} \leftarrow \operatorname{Mod}_{1}^{d} \rightarrow \operatorname{Bun}_{1}
$$

may be identified with

$$
\operatorname{Pic}(X) \leftarrow \operatorname{Pic}(X) \times X^{(d)} \rightarrow \operatorname{Pic}(X),
$$

where the left arrow is the projection on the first factor and the right arrow is the composition

$$
\operatorname{Pic}(X) \times X^{(d)} \stackrel{\text { id } \times \mathrm{AJ}}{\longrightarrow} \operatorname{Pic}(X) \times \operatorname{Pic}^{d}(X) \stackrel{\text { mult }}{\longrightarrow} \operatorname{Pic}(X) .
$$

Therefore, for $\mathcal{K} \in \mathrm{D}(\operatorname{Pic}(X))$ we have:

$$
h_{!} \rightarrow\left(h^{\leftarrow *}(\mathcal{K}) \otimes \pi^{*}\left(\mathcal{L}_{E}^{d}\right)\right) \simeq \operatorname{mult}_{!}\left(\mathcal{K} \otimes(\mathrm{AJ}) !\left(E^{(d)}\right)\right)=0,
$$

by Deligne's theorem.

\section{The construction of Aut $_{E}$}

Let $\mathcal{C o h}_{n}^{\prime}$ denote the stack classifying pairs $(\mathcal{M}, s)$, where $\mathcal{M} \in \mathcal{C o h}_{n}$ and $s$ is an injective map $\Omega^{n-1} \rightarrow \mathcal{M}$. Here $\Omega$ stands for the canonical bundle of $X$ and we write $\Omega^{k}$ for $\Omega^{\otimes k}$. We denote by $\operatorname{Bun}_{n}^{\prime}$ the preimage in $\operatorname{Coh}_{n}^{\prime}$ of the open substack $\operatorname{Bun}_{n} \subset \operatorname{Coh}_{n}$. Let $\varrho_{n}: \operatorname{Coh}_{n}^{\prime} \rightarrow \operatorname{Coh}_{n}$ be the forgetful map; we use the same notation for the forgetful map $\operatorname{Bun}_{n}^{\prime} \rightarrow \operatorname{Bun}_{n}$.

In this section, starting with a local system $E$ on $X$ of an arbitrary rank, we will construct a complex $\mathcal{S}_{E}^{\prime}$ on $\mathcal{C} o h_{n}^{\prime}$. Later we will show that if $E$ is an irreducible local system of rank $n$ which satisfies Conjecture 2.3 then $\mathcal{S}_{E}^{\prime}$ descends to a perverse sheaf $\mathcal{S}_{E}$ on $\operatorname{Coh}_{n}$. The restriction of $\mathcal{S}_{E}$ to $\mathrm{Bun}_{n}$ will then be the Hecke eigensheaf Aut $_{E}$. We present below three constructions of $\mathcal{S}_{E}^{\prime}$ (two of them in this section, and one more in the next section).

3.1. The first construction. The following is a version of the construction presented in [Lau2 FGKV].

Define an algebraic stack $\widetilde{\mathcal{Q}}$ as follows. For a $\mathbb{k}$-scheme $S, \operatorname{Hom}(S, \widetilde{\mathcal{Q}})$ is the groupoid whose objects are quadruples $\left(\mathcal{M}_{S}, \beta_{S},\left(\mathcal{M}_{i, S}^{0}\right),\left(\widetilde{s}_{i, S}\right)\right)$, where $\mathcal{M}_{S}$ is a coherent sheaf on $X \times S$ of generic rank $n, \mathcal{M}_{S}^{0}$ is a rank $n$ bundle on $X \times S$, $\beta_{S}: \mathcal{M}_{S}^{0} \rightarrow \mathcal{M}_{S}$ is an embedding of the corresponding $\mathcal{O}_{X \times S}$-modules, such that the quotient is $S$-flat, $\left(\mathcal{M}_{i, S}^{0}\right)$ is a full flag of subbundles

$$
0=\mathcal{M}_{0, S}^{0} \subset \mathcal{M}_{1, S}^{0} \subset \ldots \subset \mathcal{M}_{n-1, S}^{0} \subset \mathcal{M}_{n, s}^{0}=\mathcal{M}_{S}^{0},
$$

and $\widetilde{s}_{i, S}$ is an isomorphism $\Omega^{n-i} \nabla \mathcal{O}_{S} \simeq \mathcal{M}_{i, S}^{0} / \mathcal{M}_{i-1, S}^{0}, i=1, \ldots, n$. The morphisms are the isomorphisms of the corresponding $\mathcal{O}_{X \times S}$-modules making all diagrams commutative (we remark that in [FGKV] we used the notation $J$ instead of $\mathcal{M}^{0}$ ). 
There is a representable morphism of stacks $\widetilde{\nu}: \widetilde{Q} \rightarrow \operatorname{Coh}_{n}^{\prime}$, which for each $\mathbb{k}_{-}$ scheme $S$ maps $\left(\mathcal{M}_{S}, \beta_{S},\left(\mathcal{M}_{i, S}^{0}\right),\left(\widetilde{s}_{i, S}\right)\right)$ to the pair $\left(\mathcal{M}_{S}, \beta_{S} \circ \widetilde{s}_{1, S}\right)$, where $s_{1, S}$ is viewed as an embedding of $\Omega^{n-1} \otimes \mathcal{O}_{S}$ into $\mathcal{M}_{S}^{0}$.

We also define the morphism $\alpha: \widetilde{\mathcal{Q}} \rightarrow \operatorname{Coh}_{0}$ sending $\left(\mathcal{M}_{S}, \beta_{S},\left(\mathcal{M}_{i, S}^{0}\right),\left(\widetilde{s}_{i, S}\right)\right)$ to the sheaf $\mathcal{M}_{S} / \operatorname{Im} \beta_{S}$, and the morphism ev $: \widetilde{\mathcal{Q}} \rightarrow \mathbb{G}_{a}$ defined as follows.

Given two coherent sheaves $\mathcal{L}$ and $\mathcal{L}^{\prime}$ on $X$, consider the stack $\mathcal{E} x t^{1}\left(\mathcal{L}^{\prime}, \mathcal{L}\right)$. The objects of the groupoid $\operatorname{Hom}\left(S, \mathcal{E} x t^{1}\left(\mathcal{L}^{\prime}, \mathcal{L}\right)\right)$ are coherent sheaves $\mathcal{L}^{\prime \prime}$ on $X \times S$ together with a short exact sequence

$$
0 \rightarrow \mathcal{L} \otimes \mathcal{O}_{S} \rightarrow \mathcal{L}^{\prime \prime} \rightarrow \mathcal{L}^{\prime} \otimes \mathcal{O}_{S} \rightarrow 0,
$$

and morphisms are maps between such exact sequences inducing the identity isomorphisms at the ends. There is a canonical morphism from the stack $\mathcal{E} x t^{1}\left(\mathcal{L}^{\prime}, \mathcal{L}\right)$ to the scheme $\operatorname{Ext}^{1}\left(\mathcal{L}^{\prime}, \mathcal{L}\right)$. We have for each $i=1, \ldots, n-1$ a natural morphism $\mathrm{ev}_{i}: \widetilde{\mathcal{Q}} \rightarrow \mathcal{E} x t^{1}\left(\Omega^{i}, \Omega^{i-1}\right)$, which sends the data of $\left(\mathcal{M}_{S}, \beta_{S},\left(\mathcal{M}_{i, S}^{0}\right),\left(\widetilde{s}_{i, S}\right)\right)$ to

$$
0 \rightarrow \mathcal{M}_{i, S}^{0} / \mathcal{M}_{i-1, S}^{0} \rightarrow \mathcal{M}_{i+1, S}^{0} / \mathcal{M}_{i-1, S}^{0} \rightarrow \mathcal{M}_{i+1, S}^{0} / \mathcal{M}_{i, S}^{0} \rightarrow 0
$$

Now ev is the composition

$$
\mathrm{ev}: \widetilde{\mathcal{Q}} \rightarrow \prod_{i=1}^{n-1} \mathcal{E} x t^{1}\left(\Omega^{i}, \Omega^{i-1}\right) \rightarrow \prod_{i=1}^{n-1} \operatorname{Ext}^{1}\left(\Omega^{i}, \Omega^{i-1}\right) \rightarrow \mathbb{G}_{a}^{n-1} \stackrel{\operatorname{sum}}{\rightarrow} \mathbb{G}_{a}
$$

We fix a non-trivial character $\psi: \mathbb{F}_{q} \rightarrow \overline{\mathbb{Q}}_{\ell}$, which gives rise to the Artin-Shreier sheaf $\mathcal{J}_{\psi}$ on the additive group $\mathbb{G}_{a}$. Define the complex $\widetilde{\mathcal{W}}_{E}$ on $\widetilde{\mathcal{Q}}$ by the formula

$$
\widetilde{\mathcal{W}}_{E}:=\alpha^{*}\left(\mathcal{L}_{E}\right) \otimes \operatorname{ev}^{*}\left(\mathcal{J}_{\psi}\right) \otimes \overline{\mathbb{Q}}_{\ell}\left(\frac{\operatorname{dim}}{2}\right)[\operatorname{dim}],
$$

where dim is the dimension of the corresponding connected component of $\widetilde{\mathcal{Q}}$.

Since the morphism $\alpha$ is smooth, $\widetilde{\mathcal{W}}_{E}$ is a perverse sheaf. Finally, we define the complex $\mathcal{S}_{E}^{\prime}$ on $\operatorname{Coh}_{n}^{\prime}$ by

$$
\mathcal{S}_{E}^{\prime}:=\widetilde{\nu}_{!}\left(\widetilde{\mathcal{W}}_{E}\right) .
$$

3.2. The second construction, via Fourier transforms. This construction is due to Laumon [Lau2]. It amounts to expressing the first construction as a series of Fourier transforms. Thus, we obtain an alternative construction of the restriction of $\mathcal{S}_{E}^{\prime}$ to the preimage in $\mathcal{C}_{o} h_{n}^{\prime}$ of a certain open substack $\mathcal{C}_{n}$ of $\mathcal{C o h}_{n}$. For technical reasons, which will become clear in the course of the proof, we choose a slightly smaller open subset of $\mathrm{Bun}_{n}$ than in [Lau2].

We will need the following result:

We call a vector bundle $\mathcal{M}$ very unstable if $\mathcal{M}$ can be decomposed into a direct sum $\mathcal{M} \simeq \mathcal{M}_{1} \oplus \mathcal{M}_{2}$, such that $\mathcal{M}_{i} \neq 0$ and $\operatorname{Ext}^{1}\left(\mathcal{M}_{1}, \mathcal{M}_{2}\right)=0$. It is clear that very unstable vector bundles form a constructible subset $\mathrm{Bun}_{n}^{\text {vuns }}$ of $\mathrm{Bun}_{n}$.

Let $\mathcal{L}^{\text {est }}$ be any fixed line bundle.

3.3. Lemma. There exists an integer $c_{g, n}$ with the following property: if $d \geq c_{g, n}$ and $\mathcal{M} \in \operatorname{Bun}_{n}^{d}(\overline{\mathbb{k}})$ is such that $\operatorname{Hom}\left(\mathcal{N}, \mathcal{L}^{\text {est }}\right) \neq 0$, then $\mathcal{M}$ is very unstable.

Proof. We will prove a slightly stronger statement. Namely, for each $n$ we will find an integer $c_{g, n}$ such that for $\mathcal{M} \in \operatorname{Bun}_{n}^{d}, d \geq c_{g, n}$, with $\operatorname{Hom}\left(\mathcal{M}, \mathcal{L}^{\text {est }}\right) \neq 0$ there exists a decomposition $\mathcal{M} \simeq \mathcal{M}_{1} \oplus \mathcal{M}_{2}$ with $\mathcal{M}_{i} \neq 0$, $\operatorname{Ext}^{1}\left(\mathcal{M}_{1}, \mathcal{M}_{2}\right)=0$, and $\frac{\operatorname{deg}\left(\mathcal{M}_{2}\right)}{\operatorname{rank}\left(\mathcal{M}_{2}\right)} \geq \frac{\operatorname{deg}(\mathcal{M})}{n}$. 
Set $c_{g, 1}=\operatorname{deg}\left(\mathcal{L}^{\text {est }}\right)$. By induction, we can assume that $c_{g, i}, i \leq n-1$, satisfying the above properties have been found. Let us show that any integer $c_{g, n}$ such that

$$
\left(c_{g, n}-d^{\mathrm{est}}-(n-1)(2 g-2)\right) \cdot \frac{i}{n-1}>c_{g, i}, \quad \forall i=1, \ldots, n-1,
$$

will do.

Indeed, let $c_{g, n}$ be such an integer. Suppose that for some $\mathcal{M} \in \operatorname{Bun}_{n}^{d}, d \geq c_{g, n}$, we have $\operatorname{Hom}\left(\mathcal{M}, \mathcal{L}^{\text {est }}\right) \neq 0$. Then there exists a short exact sequence

$$
0 \rightarrow \mathcal{M}^{\prime} \rightarrow \mathcal{M} \rightarrow \mathcal{L}^{\prime} \rightarrow 0
$$

where $\mathcal{L}^{\prime}$ is a line bundle such that $\operatorname{Hom}\left(\mathcal{L}^{\prime}, \mathcal{L}^{\text {est }}\right) \neq 0$. By $(3.3)$ we have: $\frac{\operatorname{deg}\left(\mathcal{M}^{\prime}\right)}{n-1} \geq$ $\frac{\operatorname{deg}(\mathcal{M})}{n}$. Hence, if $\operatorname{Ext}^{1}\left(\mathcal{L}^{\prime}, \mathcal{M}^{\prime}\right)$ vanishes, the decomposition $\mathcal{M} \simeq \mathcal{M}^{\prime} \oplus \mathcal{L}^{\prime}$ satisfies our requirements and we are done. Thus, it remains to consider the case $\operatorname{Ext}^{1}\left(\mathcal{L}^{\prime}, \mathcal{M}^{\prime}\right) \neq 0$. Then, by Serre duality, we obtain that $\operatorname{Hom}\left(\mathcal{M}^{\prime} \otimes \Omega^{-1}, \mathcal{L}^{\prime}\right) \neq 0$ and hence $\operatorname{Hom}\left(\mathcal{M}^{\prime} \otimes \Omega^{-1}, \mathcal{L}^{\text {est }}\right) \neq 0$. From the definition of $\mathcal{M}^{\prime}$ and $(3.3)$ we conclude that $\operatorname{deg}\left(\mathcal{M}^{\prime} \otimes \Omega^{-1}\right)>c_{g, n-1}$. We first observe that this forces $n>2$. For $n=2$ we get $\operatorname{deg}\left(\mathcal{M}^{\prime} \otimes \Omega^{-1}\right)>\operatorname{deg}\left(\mathcal{L}^{\text {est }}\right)$, forcing $\operatorname{Hom}\left(\mathcal{M}^{\prime} \otimes \Omega^{-1}, \mathcal{L}^{\text {est }}\right)$ to vanish, a contradiction.

Using our induction hypothesis, we can find a direct sum decomposition $\mathcal{M}^{\prime} \otimes$ $\Omega^{-1} \simeq \mathcal{M}_{1}^{\prime} \oplus \mathcal{M}_{2}^{\prime}$ with $\operatorname{Ext}^{1}\left(\mathcal{M}_{1}^{\prime}, \mathcal{M}_{2}^{\prime}\right)=0$ and $\frac{\operatorname{deg}\left(\mathcal{M}_{2}^{\prime}\right)}{i} \geq \frac{\operatorname{deg}\left(\mathcal{M}^{\prime} \otimes \Omega^{-1}\right)}{n-1}$, where $i=$ $\operatorname{rank}\left(\mathcal{M}_{2}^{\prime}\right)$. Moreover, without loss of generality we can assume that $\mathcal{M}_{2}^{\prime}$ admits no further decomposition satisfying the above condition (indeed, if it does, we simply split $\mathcal{M}_{2}^{\prime}$ further $)$. Since $\operatorname{deg}\left(\mathcal{M}_{2}^{\prime}\right) \geq \operatorname{deg}\left(\mathcal{M}^{\prime} \otimes \Omega^{-1}\right) \cdot \frac{i}{n-1} \geq c_{g, i}, \operatorname{Hom}\left(\mathcal{M}_{2}^{\prime}, \mathcal{L}^{\text {est }}\right)$ must vanish by the induction hypothesis.

By Serre duality, it follows that $\operatorname{Ext}^{1}\left(\mathcal{L}^{\text {est }}, \mathcal{M}_{2}^{\prime} \otimes \Omega\right)=0$, and hence $\mathcal{M}_{2}:=\mathcal{M}_{2}^{\prime} \otimes \Omega$ is a direct summand in $\mathcal{M}$. More precisely, $\mathcal{M} \simeq \mathcal{M}_{2} \oplus \mathcal{M}_{1}$, where $\mathcal{M}_{1}$ fits into a short exact sequence

$$
0 \rightarrow \mathcal{M}_{1}^{\prime} \otimes \Omega \rightarrow \mathcal{M}_{1} \rightarrow \mathcal{L}^{\prime} \rightarrow 0
$$

Therefore, $\operatorname{Ext}^{1}\left(\mathcal{M}_{1}, \mathcal{M}_{2}\right)=0$ and

$$
\frac{\operatorname{deg}\left(\mathcal{M}_{2}\right)}{\operatorname{rank}\left(\mathcal{M}_{2}\right)} \geq \frac{\operatorname{deg}\left(\mathcal{M}^{\prime}\right)}{n-1} \geq \frac{\operatorname{deg}(\mathcal{M})}{n}
$$

This completes the proof.

Notational convention. For notational convenience, by degree of a coherent sheaf of generic rank $k$ we will understand its usual degree $-k(k-1)(g-1)$, so that the bundle $\mathcal{O} \oplus \Omega \oplus \ldots \oplus \Omega^{k-1}$ is of degree zero.

To define $\mathcal{C}_{n}$, we choose the line bundle $\mathcal{L}^{\text {est }}$ of a sufficiently large degree such that for any bundle $\mathcal{M}$ on $X$ of rank $k \leq n, \operatorname{Hom}\left(\mathcal{M}, \mathcal{L}^{\text {est }}\right)=0$ implies that

(a) $\operatorname{deg}(\mathcal{M})>n k(2 g-2)$,

(b) $\operatorname{Ext}^{1}\left(\Omega^{k-1}, \mathcal{M}\right)=0$.

For example, any line bundle $\mathcal{L}^{\text {est }}$ of degree $>(2 n+2)(g-1)$ will do.

Thus, let $c_{g, n}$ be an integer satisfying the requirements of Lemma 3.3. For $d \geq c_{g, n}$, let $\mathcal{C}_{k}^{d}$ be the open substack of $\operatorname{Coh}_{k}^{d}$ consisting of $\mathcal{M} \in \mathcal{C o h}_{k}^{d}$ such that $\operatorname{Hom}\left(\mathcal{M}, \mathcal{L}^{\text {est }}\right)=0$. Finally, we set $\mathcal{C}_{k}=\bigcup_{d \geq c_{g, n}} \mathcal{C}_{k}^{d}$.

Note that by construction any $\mathcal{M} \in\left(\operatorname{Bun}_{n}^{d}-\mathcal{C}_{n}^{d} \cap \operatorname{Bun}_{n}^{d}\right)(\overline{\mathbb{k}})$, for $d \geq c_{g, n}$, is very unstable. This property of $\mathcal{C}_{n}$ will be crucial in Sect. 6 


\subsection{The fundamental diagram. Let}

$\mathcal{E}_{k}=$ the stack classifying pairs $\left(\mathcal{M}_{k}, s_{k}\right), \mathcal{M}_{k} \in \mathcal{C}_{k}, s_{k} \in \operatorname{Hom}\left(\Omega^{k-1}, \mathcal{M}_{k}\right)$,

$\mathcal{E}_{k}^{\vee}=$ the stack classifying extensions $0 \rightarrow \Omega^{k} \rightarrow \mathcal{M}_{k+1} \rightarrow \mathcal{M}_{k} \rightarrow 0$, with $\mathcal{M}_{k} \in \mathcal{C}_{k}$.

We have natural projections $\rho_{k}: \mathcal{E}_{k} \rightarrow \mathcal{C}_{k}$ and $\rho_{k}^{\vee}: \mathcal{E}_{k}^{\vee} \rightarrow \mathcal{C}_{k}$, which form dual vector bundles over $\mathcal{C}_{k}$, due to the above conditions on $\mathcal{L}^{\text {est }}$. We have: $\rho_{k}=\varrho_{k} \mid \varepsilon_{k}$.

Next, we set:

$$
\begin{aligned}
\mathcal{E}_{k}^{0} & =\left\{\left(\mathcal{M}_{k}, s_{k}\right) \in \mathcal{E}_{k} \mid s_{k} \text { is injective }\right\} \subset \mathcal{E}_{k}, \\
\mathcal{E}_{k}^{\vee 0} & =\left\{\left(0 \rightarrow \Omega^{k} \rightarrow \mathcal{M}_{k+1} \rightarrow \mathcal{M}_{k} \rightarrow 0\right) \in \mathcal{E}_{k}^{\vee} \mid \mathcal{M}_{k+1} \in \mathcal{C}_{k+1}\right\} \subset \mathcal{E}_{k}^{\vee} .
\end{aligned}
$$

Clearly, $\mathcal{E}_{k}^{0} \simeq \mathcal{E}_{k-1}^{\vee 0}$. Denote by $j_{k}$ the embedding $\mathcal{E}_{k}^{0} \hookrightarrow \mathcal{E}_{k}$. Note also that $\mathcal{E}_{k}^{0}$ is an open substack in $\mathrm{Coh}_{n}^{\prime}$.

Consider the following diagram:

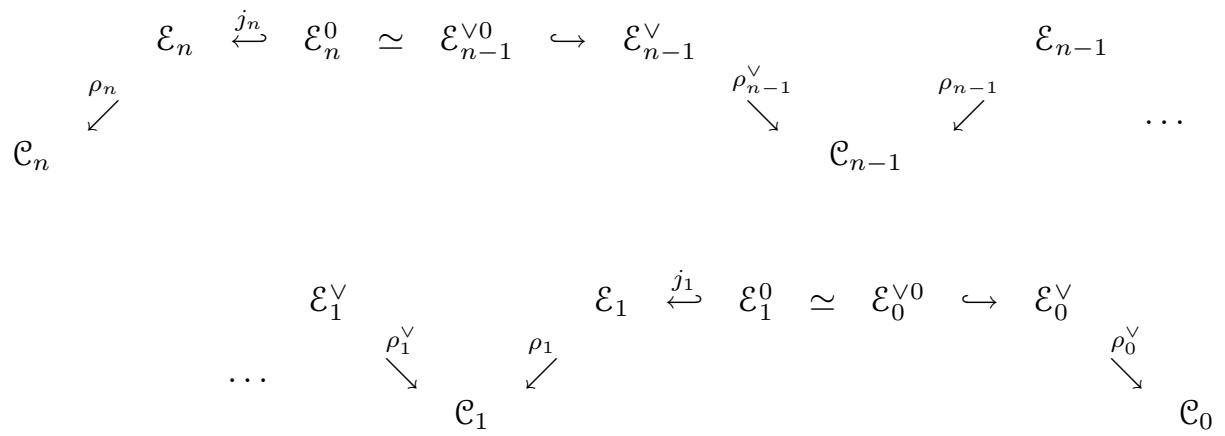

We set $\mathcal{F}_{E, 1}$ to be a complex on $\mathcal{E}_{1}^{0}$ equal to the pull-back of Laumon's sheaf $\mathcal{L}_{E}$ under

$$
\mathcal{E}_{1}^{0} \simeq \mathcal{E}_{0}^{\vee 0} \hookrightarrow \mathcal{E}_{0}^{\vee} \stackrel{\rho_{0}^{\vee}}{\longrightarrow} \mathcal{C}_{0} \simeq \operatorname{Coh}_{0} .
$$

Since $\rho_{0}^{\vee}$ is a smooth morphism and $\mathcal{E}_{1}^{0} \rightarrow \mathcal{E}_{0}^{\vee}$ is an open embedding, the restriction of $\mathcal{F}_{E, 1}[d]$ to the connected component of $\mathcal{E}_{1}^{0}$ corresponding to coherent sheaves of degree $d$ is a perverse sheaf.

Next, we define the complexes $\mathcal{F}_{E, k}$ on $\mathcal{E}_{k}^{0}$ by the formula:

$$
\mathcal{F}_{E, k+1}=\left.\operatorname{Four}\left(j_{k !}\left(\mathcal{F}_{E, k}\right)\right)\right|_{\mathcal{E}_{k+1}^{0}},
$$

where Four is the Fourier transform functor.

Unraveling the second construction we obtain (see [Lau2]):

3.5. Lemma. The complex $\mathcal{F}_{E, n}$ coincides (up to a cohomological shift and Tate's twist) with the restriction of $\mathcal{S}_{E}^{\prime}$ to $\mathcal{E}_{n}^{0} \subset \operatorname{Coh}_{n}^{\prime}$.

3.6. The cleanness property of $\mathcal{F}_{E, k}$. Let us now assume that $E$ is an irreducible rank $n$ local system and that Conjecture 2.3 holds for $E$.

In Sect. 5 we prove the following theorem, which was conjectured by Laumon in Lau2, Exposé I, Conjecture 3.2.

3.7. Theorem. For $k=1, \ldots, n-1$, the canonical maps $j_{k !}\left(\mathcal{F}_{E, k}\right) \rightarrow j_{k *}\left(\mathcal{F}_{E, k}\right)$ are isomorphisms. 
Recall that a complex $\mathcal{K}$ on $Y$ is called clean with respect to an embedding $Y \stackrel{j}{\hookrightarrow} \bar{Y}$ if $j_{!}(\mathcal{K}) \rightarrow j_{*}(\mathcal{K})$ is an isomorphism, i.e., $\left.j_{*}(\mathcal{K})\right|_{\bar{Y}-Y}=0$. When $\mathcal{K}$ is a perverse sheaf, cleanness implies that $j_{!}(\mathcal{K}) \simeq j_{! *}(\mathcal{K}) \simeq j_{*}(\mathcal{K})$. In this language Theorem 3.7 states that the sheaf $\mathcal{F}_{E, k}$ on $\mathcal{E}_{k}^{0}$ is clean with respect to $j_{k}: \mathcal{E}_{k}^{0} \hookrightarrow \mathcal{E}_{k}$.

By construction, Laumon's sheaf $\mathcal{L}_{E}^{d}$ is perverse and irreducible. As was mentioned earlier, the restriction of $\mathcal{F}_{E, 1}$ to each connected component of $\mathcal{E}_{1}^{0}$ is, therefore, also an irreducible perverse sheaf, up to a cohomological shift. Since the Fourier transform functor preserves perversity and irreducibility, we obtain by induction:

3.8. Corollary. The restriction of $\mathcal{F}_{E, n}$ to each connected component of $\mathcal{E}_{n}^{0}$ is an irreducible perverse sheaf, up to a cohomological shift.

In Sect. 6 we will derive from Corollary 3.8 the following theorem, which was conjectured by Laumon in Lau2], Exposé I, Conjecture 3.1. Denote by $\rho_{n}^{0}$ the morphism $\mathcal{E}_{n}^{0} \rightarrow \mathcal{C}_{n}$ obtained by restriction from $\rho_{n}$.

3.9. Theorem. The complex $\mathcal{F}_{E, n}$ descends to $\mathcal{C}_{n}$, i.e., there exists a perverse sheaf $\mathcal{S}_{E}^{0}$ on $\mathcal{C}_{n}$, such that

$$
\mathcal{F}_{E, n} \otimes \overline{\mathbb{Q}}_{\ell}\left(\frac{n^{2} \cdot(g-1)}{2}\right)\left[n^{2} \cdot(g-1)\right] \simeq \rho_{n}^{0 *}\left(\mathcal{S}_{E}^{0}\right) .
$$

Moreover, the restriction of $\mathcal{S}_{E}^{0}$ to each connected component of $\mathcal{C}_{n}$ is a non-zero irreducible perverse sheaf.

3.10. A summary. Above we have described two constructions of a Hecke eigensheaf associated to a local system $E$. In the next section we will describe the third construction. Before doing that, we wish to summarize the relations between the three constructions and to indicate the strategy of the proof of the main result of this paper that the Vanishing Conjecture 2.3 for $E$ implies the geometric Langlands Conjecture 1.3 .

In the first construction given in Sect. 3.1 we constructed a sheaf $\mathcal{S}_{E}^{\prime}$ on the moduli stack $\operatorname{Coh}_{n}^{\prime}$ of pairs $\left(\mathcal{M}, \Omega^{n-1} \hookrightarrow \mathcal{M}\right)$, where $\mathcal{M}$ is a coherent sheaf on $X$ of generic rank $n$.

In the second construction given in Sect. 3.2 we defined a sheaf $\mathcal{F}_{E, n}$ on an open substack $\mathcal{E}_{n}^{0}$ of $\operatorname{Coh}_{n}^{\prime}$ (recall that this open substack is the preimage of $\mathcal{C}_{n} \subset \operatorname{Coh}_{n}$ under $\left.\varrho_{n}: \operatorname{Coh}_{n}^{\prime} \rightarrow \operatorname{Coh}_{n}\right)$.

Finally, in the third construction given in the next section we produce a sheaf Aut $_{E}^{\prime}$ on the stack $\operatorname{Bun}_{n}^{\prime}$ of pairs $\left(\mathcal{M}, \Omega^{n-1} \hookrightarrow \mathcal{M}\right)$, where $\mathcal{M}$ is a rank $n$ vector bundle on $X$ (obviously, $\operatorname{Bun}_{n}^{\prime}$ is the preimage $\left.\varrho_{n}^{-1}\left(\operatorname{Bun}_{n}\right) \subset \operatorname{Coh}_{n}^{\prime}\right)$.

The relations between these sheaves are as follows:

$$
\left.\mathcal{S}_{E}^{\prime}\right|_{\varepsilon_{n}^{0}} \simeq \mathcal{F}_{E, n}
$$

and

$$
\left.\mathcal{S}_{E}^{\prime}\right|_{\mathrm{Bun}_{n}^{\prime}} \simeq \mathrm{Aut}_{E}^{\prime},
$$

up to cohomological shifts and Tate's twists. These isomorphisms are established in Lemma 3.5 and Lemma 4.4 respectively. In particular,

$$
\left.\left.\mathcal{F}_{E, n}\right|_{\varrho^{-1}\left(\operatorname{Bun}_{n} \cap \mathcal{C}_{n}\right)} \simeq \operatorname{Aut}_{E}^{\prime}\right|_{\varrho^{-1}\left(\operatorname{Bun}_{n} \cap \mathcal{C}_{n}\right)} .
$$


Thus, the primary role of the first construction is to establish a link between the second and the third ones.

Our first goal is to prove Corollary 3.8 that if $E$ is irreducible, then $\mathcal{F}_{E, n}$ (obtained as the result of the second construction) is irreducible and perverse when restricted to each connected component (up to a cohomological shift and a twist). This complex is obtained by iterating the operations of Fourier transform, which are known to preserve irreducibility and perversity, and of !-extensions with respect to the open embeddings $j_{k}$. Hence we need to show that the !-extensions of the intermediate sheaves $\mathcal{F}_{E, k}$ coincide with their Goresky-MacPherson extensions, i.e., that $\mathcal{F}_{E, k}$ are clean with respect to $j_{k}$. This is the content of Theorem 3.7 which is derived in Sect. 5 from the Vanishing Conjecture 2.3.

Next, we will prove that $\mathcal{F}_{E, n}$ descends to a perverse sheaf on the open subset $\mathcal{C}_{n} \cap \operatorname{Bun}_{n} \subset \mathcal{C}_{n}$ under a natural smooth morphism $\rho_{n}^{0}: \mathcal{E}_{n}^{0} \rightarrow \mathcal{C}_{n}$ (see Theorem 3.9). This will be done using the perversity and irreducibility of $\mathcal{F}_{E, n}$ and some information about the Euler characteristics of the stalks of the sheaf Aut ${ }_{E}^{\prime}$. In order to compute these Euler characteristics, we use the third construction (and its relation to the second construction) in an essential way.

Having obtained the perverse sheaf $\mathcal{S}_{E}^{0}$ on $\mathcal{C}_{n} \cap \mathrm{Bun}_{n}$, we take its GoreskyMacPherson extension to the union of those connected components of $\mathrm{Bun}_{n}$ which have a non-empty intersection with $\mathcal{C}_{n}$, i.e., to $\bigcup_{d>c_{g, n}} \operatorname{Bun}_{n}^{d}$. This gives us a perverse sheaf $\mathrm{Aut}_{E}$ on this stack, irreducible on each connected component.

Then we prove that $\mathrm{Aut}_{E}$ may be extended to the entire stack Bun ${ }_{n}$ in such a way that it is a Hecke eigensheaf. We give two independent proofs of this statement, in Sect. 7 and Sect. 8 , using the third and the second constructions, respectively. Finally, in Sect. 9 we prove that the Hecke property of Aut $_{E}$ and Conjecture 2.3 imply that $\mathrm{Aut}_{E}$ is automatically cuspidal.

This summarizes the main steps in our proof of Conjecture 1.3. In addition, we will prove the following result. Let us denote by $\mathcal{S}_{E}$ the Goresky-MacPherson extension of $\mathrm{Aut}_{E}$ from $\mathrm{Bun}_{n}$ to $\mathrm{Coh}_{n}$. In Sect. 8 we will show that the sheaves $\varrho_{n}^{*}\left(\mathcal{S}_{E}\right)$ and $\mathcal{S}_{E}^{\prime}$ on $\operatorname{Coh}_{n}^{\prime}$ are isomorphic, up to a cohomological shift and Tate's twist. The same is true for the sheaves $\varrho_{n}^{*}\left(\mathrm{Aut}_{E}\right)$ and $\mathrm{Aut}_{E}^{\prime}$ on $\mathrm{Bun}_{n}^{\prime}$.

Note, however, that neither $\mathcal{S}_{E}^{\prime}$ nor $\left.\operatorname{Aut}_{E}^{\prime} \simeq \mathcal{S}_{E}^{\prime}\right|_{\operatorname{Bun}_{n}^{\prime}}$ is perverse on the entire stack $\operatorname{Coh}_{n}^{\prime}$ and $\mathrm{Bun}_{E}^{\prime}$, respectively. This does not contradict the above assertions: although the morphism $\varrho_{n}$ is smooth over $\mathcal{C}_{n}$, it is not smooth over the entire $\operatorname{Coh}_{n}$.

\section{The COnstruction Via the Whittaker SheaF}

In this section we will present another construction of the sheaf $\mathcal{S}_{E}^{\prime}$ (more precisely, of its restriction to $\left.\mathrm{Bun}_{n}^{\prime}\right) 11$ Conceptually, this construction should be viewed as a geometric counterpart of the construction of automorphic functions for $G L_{n}$ from the Whittaker functions due to Piatetskii-Shapiro [PS1] and Shalika Sha] (see FGKV and Sect. 4.13 below for more details).

4.1. Drinfeld's compactification. We introduce the stack $\bar{Q}$, which classifies the data $\left(\mathcal{M},\left(s_{i}\right)\right)$, where $\mathcal{M}$ is a rank $n$ bundle and $s_{i}, i=1, \ldots, n$, are injective

\footnotetext{
${ }^{1}$ This construction was independently found by I. Mirković.
} 
homomorphisms of coherent sheaves

$$
\begin{aligned}
\Omega^{n-1} & \stackrel{s_{1}}{\longrightarrow} \mathcal{M}, \\
\Omega^{(n-1)+(n-2)} & \stackrel{s_{2}}{\longrightarrow} \Lambda^{2} \mathcal{N}, \\
& \cdots \\
\Omega^{\frac{n(n-1)}{2}} & \stackrel{s_{n-1}}{\longrightarrow} \Lambda^{n-1} \mathcal{N}, \\
\Omega^{\frac{n(n-1)}{2}} & \stackrel{s_{n}}{\longrightarrow} \Lambda^{n} \mathcal{M}
\end{aligned}
$$

satisfying the requirement that at the generic point of $X$ the collection $\left(s_{i}\right)$ defines a complete flag of subbundles in $\mathcal{M}$ (see [FGV]).

In concrete terms, this requirement may be phrased as follows: the transposed maps $s_{i}^{*}: \Lambda^{i} \mathcal{N}^{*} \rightarrow \Omega^{-(n-1)-\ldots-(n-i)}$ should satisfy the Plücker relations

$$
\left(s_{p}^{*} \otimes s_{q}^{*}\right)\left(\Phi_{p, q}^{k}\right)=0, \quad 1 \leq k \leq p \leq q \leq n-1 .
$$

Here $\Phi_{p, q}^{k}$ is the subsheaf of $\Lambda^{p} \mathcal{M} \otimes \Lambda^{q} \mathcal{M}$ spanned by elements of the form

$$
\begin{aligned}
\left(v_{1} \wedge \ldots \wedge v_{q}\right) & \otimes\left(w_{1} \wedge \ldots \wedge w_{q}\right) \\
& -\sum_{i_{1}<\ldots<i_{k}}\left(v_{1} \ldots w_{1} \ldots w_{k} \ldots v_{p}\right) \otimes\left(v_{i_{1}} \ldots v_{i_{k}} \wedge w_{k+1} \ldots w_{q}\right)
\end{aligned}
$$

(i.e., we exchange $k$-tuples of elements of the set $\left\{v_{i}\right\}$ with the first $k$ elements of the set $\left\{w_{j}\right\}$ preserving the order).

To motivate this definition, denote by $\operatorname{Fl}(V)$ the variety of full flags in an $n^{-}$ dimensional vector space $V$ over $\mathbb{k}$. We have a natural embedding

$$
\left(s_{1}, \ldots, s_{n-1}\right): \operatorname{Fl}(V) \hookrightarrow \prod_{i=1}^{n-1} \mathbb{P} \Lambda^{i} V .
$$

According to the results of $[\mathrm{T}]$ and $[\mathrm{Fu}]$, Sect. 9.1, the ideal of the image of $\mathrm{Fl}(V)$ under this map is generated by the elements of the form (4.3) 2

Thus, if all of the above homomorphisms

$$
s_{i}: \Omega^{(n-1)+\ldots+(n-i)} \rightarrow \Lambda^{i} \mathcal{M}, \quad i=1, \ldots, n-1,
$$

are maximal embeddings (i.e., bundle maps), then the data of $\left(\mathcal{M},\left(s_{i}\right)\right)$ determine a full flag of subbundles of $\mathcal{M}$. We denote the open substack of $\bar{Q}$ classifying the data $\left(\mathcal{M},\left(s_{i}\right)\right)$ satisfying this condition by $\mathcal{Q}$ and the open embedding $\mathcal{Q} \hookrightarrow \bar{Q}$ by $j$.

We will denote by $\overline{\mathbf{Q}}^{d}$ (resp., $\mathcal{Q}^{d}, \widetilde{Q}^{d}$ ) the connected component of $\overline{\mathrm{Q}}$ (resp., Q, $\widetilde{Q})$ corresponding to vector bundles $\mathcal{N}$ of degree $d$ (recall our notational convention from Sect. 3.2). Note that $\operatorname{dim}\left(\overline{\mathrm{Q}}^{d}\right)=d n+\operatorname{dim}\left(\overline{\mathrm{Q}}^{0}\right)$.

There is a morphism

$$
\tau: \overline{\mathrm{Q}}^{d} \longrightarrow X^{(d)}
$$

sending $\left(\mathcal{M},\left(s_{i}\right)\right)$ to $D$, the divisor of zeros of the last map $s_{n}: \Omega^{\frac{n(n-1)}{2}} \longrightarrow \Lambda^{n} M$ in (4.1). Denote by $\bar{Q}^{D}$ (resp., $Q^{D}$ ) the preimage of $D$ under $\tau$ in $\bar{Q}^{d}$ (resp., $Q^{D}$ ).

\footnotetext{
${ }^{2}$ If char $\mathbb{k}=0$, then the generators with $k=1$ suffice, but not always so if char $\mathbb{k}>0$; see $[\mathrm{T}]$.
} 
4.2. Remark. The stack $\overline{\mathbf{Q}}^{D}$ is the stack $\overline{\mathrm{Bun}}_{N}^{\mathcal{F}_{T}}$ defined in [FGV], Sect. 2.2.2, where $\mathcal{F}_{T}$ is the $T$-bundle on $X$, which corresponds to the $n$-tuple of line bundles $\left(\Omega^{n-1}, \ldots, \Omega, \mathcal{O}(D)\right)$. The stack $Q^{D}$ is the stack $\operatorname{Bun}_{N}^{\mathcal{F}_{T}}$ from [FGV], Sect. 2.2.1.

We recall from $\left[\mathrm{FGV}, \mathrm{BG}\right.$ that the Drinfeld compactification $\overline{\mathrm{Bun}}_{N}^{\mathcal{F}_{T}}$ classifies the data $\left(\mathcal{M},\left(\kappa^{\check{\lambda}}\right)\right)$, where $\check{\lambda}$ runs over the set of dominant weights of $G L_{n}$. Further, $\kappa^{\check{\lambda}}$ is a homomorphism of coherent sheaves $\mathcal{L}_{\mathcal{F}_{T}}^{\check{\lambda}} \rightarrow \mathcal{V}_{\mathcal{F}_{T}}^{\check{\lambda}}$, where $\mathcal{L}_{\mathcal{F}_{T}}^{\check{L}}$ is the line bundle $\mathcal{F}_{T} \underset{T}{\times} \check{\lambda}$, and $\mathcal{V}_{\mathcal{F}_{T}}^{\check{L}}$ is the vector bundle corresponding to $\mathcal{M}$ and the Weyl representation of $G L_{n}$ of highest weight $\lambda$. In addition, the homomorphisms $\kappa^{\check{\lambda}}$ have to satisfy a set of Plücker type relations described in Sect. 2.2.2 of [FGV]. These relations determine all $\kappa^{\check{\lambda}}$ s from $s_{i}:=\kappa^{\check{\omega}_{i}}, i=1, \ldots, n-1$. Equivalently, these relations may be described in the form (4.2).

4.3. The Whittaker sheaf. Observe that the substack $Q^{d}$ of $\overline{\mathrm{Q}}^{d}$ embeds as an open substack into $\widetilde{Q}^{d}$, which classifies those quadruples $\left(\mathcal{M}, \beta,\left(\mathcal{M}_{i}^{0}\right),\left(s_{i}\right)\right)$, for which $\mathcal{M}$ is torsion-free and $\mathcal{N}_{i}^{0} \hookrightarrow \mathcal{M}$ are maximal embeddings (i.e., bundle maps) for $i=1, \ldots, n-1$. Recall the morphism ev $: \widetilde{Q} \rightarrow \mathbb{G}_{a}$. Denote its restriction to $\mathrm{Q}^{d} \subset \widetilde{\mathcal{Q}}^{d}$ also by ev. First, we define the complex $\Psi^{0}$ on $\overline{\mathrm{Q}}^{0}$ as

$$
\Psi^{0}=j_{!} \operatorname{ev}^{*}\left(\mathcal{J}_{\psi}\right) \otimes \overline{\mathbb{Q}}_{\ell}\left(\frac{\operatorname{dim}\left(Q^{0}\right)}{2}\right)\left[\operatorname{dim}\left(Q^{0}\right)\right] .
$$

We have a natural morphism $q: \overline{\mathcal{Q}} \rightarrow \operatorname{Bun}_{n}$ taking $\left(\mathcal{M},\left(s_{i}\right)\right)$ to $\mathcal{M}$. Recall the stack $\operatorname{Mod}_{n}^{d}$ from Sect. 2.2 and consider the Cartesian product

$$
Z^{d}:=\overline{\mathrm{Q}}^{0} \underset{\operatorname{Bun}_{n}}{\times} \operatorname{Mod}_{n}^{d} .
$$

Let ${ }^{\prime} h \rightarrow: Z^{d} \rightarrow \overline{\mathcal{Q}}^{d}$ be the morphism that sends $\left(\mathcal{M},\left(s_{i}\right), \mathcal{M}^{\prime}, \beta: \mathcal{M} \rightarrow \mathcal{M}^{\prime}\right)$ to $\left(\mathcal{M}^{\prime},\left(s_{i}^{\prime}\right)\right)$, where $s_{i}^{\prime}$ is the composition

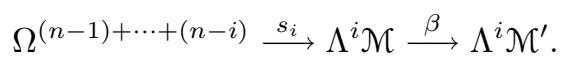

It is clear that ' $h \rightarrow$ is a proper morphism of stacks, which makes the following diagram commutative:

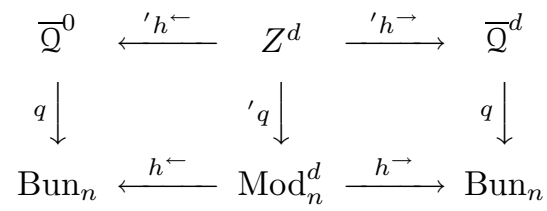

The Whittaker sheaf $\mathcal{W}_{E}^{d}$ on $\overline{\mathrm{Q}}^{d}$ is defined by the formula ${ }^{3}$

$$
\mathcal{W}_{E}^{d}:={ }^{\prime} h_{!} \rightarrow\left({ }^{\prime} h^{\leftarrow *}\left(\Psi^{0}\right) \otimes\left(\pi \circ^{\prime} q\right)^{*}\left(\mathcal{L}_{E}^{d}\right)\right) \otimes \overline{\mathbb{Q}}_{\ell}\left(\frac{d \cdot n}{2}\right)[d \cdot n] .
$$

In other words, $\mathcal{W}_{E}^{0} \simeq \Psi^{0}$, and in general $\mathcal{W}_{E}^{d}$ is obtained from $\mathcal{W}_{E}^{0}$ via a $\overline{\mathrm{Q}}$-version of the averaging functor $\mathrm{H}_{n, E}^{d}$. Namely, define the functor ' ${ }^{\prime} \mathrm{H}_{n, E}^{d}: \mathrm{D}\left(\overline{\mathrm{Q}}^{0}\right) \rightarrow \mathrm{D}\left(\overline{\mathrm{Q}}^{d}\right)$

\footnotetext{
${ }^{3}$ In the case of $G L_{2}$ this sheaf was studied in Ly1.
} 
by the formula

$$
\mathcal{K} \mapsto{ }^{\prime} h_{!} \rightarrow\left({ }^{\prime} h^{\leftarrow *}(\mathcal{K}) \otimes\left(\pi \circ^{\prime} q\right)^{*}\left(\mathcal{L}_{E}^{d}\right)\right) \otimes \overline{\mathbb{Q}}_{\ell}\left(\frac{d \cdot n}{2}\right)[d \cdot n] .
$$

Then $\mathcal{W}_{E}^{d}={ }^{\prime} \mathrm{H}_{n, E}^{d}\left(\Psi^{0}\right)$.

Let $\mathcal{W}_{E}$ be the complex on $\overline{\mathcal{Q}}$, whose restriction to $\overline{\mathcal{Q}}^{d}$ equals $\mathcal{W}_{E}^{d}$. Denote by $\nu: \overline{\mathcal{Q}} \rightarrow \operatorname{Bun}_{n}^{\prime}$ the morphism which sends $\left(\mathcal{M},\left(s_{i}\right)\right)$ to $\left(\mathcal{M}, s_{1}: \Omega^{n-1} \rightarrow \mathcal{M}\right)$. Set

$$
\operatorname{Aut}_{E}^{\prime}:=\nu_{!}\left(\mathcal{W}_{E}\right) \text {. }
$$

4.4. Lemma. The complex Aut $_{E}^{\prime}$ is canonically isomorphic to the restriction of $\mathcal{S}_{E}^{\prime}$ from $\operatorname{Coh}_{n}^{\prime}$ to $\operatorname{Bun}_{n}^{\prime}$.

Proof. Let $\widetilde{Q}_{t f}^{d}$ be the locus in $\widetilde{Q}^{d}$ corresponding to those data $\left(\mathcal{M}, \beta,\left(\mathcal{M}_{i}^{0}\right),\left(\widetilde{s}_{i}\right)\right)$ for which $\mathcal{M}$ is torsion-free. Observe that $\stackrel{\circ}{Z}^{d}:=\left({ }^{\prime} h^{\leftarrow}\right)^{-1}\left(\mathcal{Q}^{0}\right) \subset Z^{d}$ is canonically identified with $\widetilde{Q}_{t f}^{d}$. Since $\Psi^{0}$ is extended by zero from $Q^{0}$ to $\bar{Q}^{0}$, the proposition follows from the commutativity of the diagram

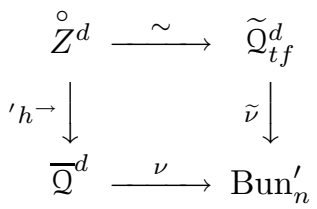

which is verified directly from the definitions.

4.5. The structure of $\mathcal{W}_{E}^{d}$. In the rest of this section we describe the structure of the Whittaker sheaf using the results of our previous work [FGV]. Strictly speaking, these results are not necessary in our proof of the geometric Langlands Conjecture 1.3. They are used only in the proof of the Hecke eigensheaf property presented in Sects. 7.2 7.6, for which we give an alternative proof in Sect. 8, which does not use the Whittaker sheaf.

To state our results, we recall first that the substack $Q^{d}$ of $\bar{Q}^{d}$ embeds as an open substack into $\widetilde{Q}^{d}$ and the composition $\mathcal{Q}^{d} \rightarrow \overline{\mathcal{Q}}^{d} \stackrel{\alpha}{\rightarrow} \operatorname{Coh}_{0}^{d}$ takes values in $\operatorname{Coh}^{\mathrm{r}, d}$ (see Sect. 2.6 for this notation). Hence,

$$
\left.\left.\left.\mathcal{W}_{E}^{d}\right|_{\mathcal{Q}^{d}} \simeq \widetilde{\mathcal{W}}_{E}^{d}\right|_{\mathcal{Q}^{d}} \simeq \tau^{*}\left(E^{(d)}\right)\right|_{Q^{d}} \otimes \operatorname{ev}^{*}\left(\mathcal{J}_{\psi}\right) \otimes \overline{\mathbb{Q}}_{\ell}\left(\frac{\operatorname{dim}\left(\mathcal{Q}^{d}\right)}{2}\right)\left[\operatorname{dim}\left(\mathcal{Q}^{d}\right)\right]
$$

where $\tau$ is the morphism in 4.4).

We will prove the following statement:

4.6. Proposition. The complex $\mathcal{W}_{E}^{d}$ on $\overline{\mathcal{Q}}^{d}$ is an irreducible perverse sheaf which is the Goresky-MacPherson extension of its restriction to $\mathbf{Q}^{d}$.

The first step in the proof of Proposition 4.6 is provided by the following result of [FGV]:

4.7. Lemma. The canonical map $\Psi^{0} \rightarrow j_{!_{*}} j^{*}\left(\Psi^{0}\right)$ is an isomorphism.

In other words, the perverse sheaf $\Psi^{0}$ on $Q^{0}$ is clean with respect to $j: Q^{0} \hookrightarrow \overline{\mathcal{Q}}^{0}$. Therefore, since $\mathcal{W}_{E}^{d} \simeq{ }^{\prime} \mathrm{H}_{n, E}^{d}\left(j_{!_{*}} j^{*}\left(\Psi^{0}\right)\right.$ ) (cf. formula 4.7), we obtain that

$$
\mathbb{D}\left(\mathcal{W}_{E}^{d}\right) \simeq \mathcal{W}_{E^{*}}^{d}
$$

where $\mathbb{D}$ is the Verdier duality functor, and $E^{*}$ is the dual local system to $E$. 
Thus, $\mathcal{W}_{E}^{d}$ is Verdier self-dual up to replacing $E$ by $E^{*}$ and to prove Proposition 4.6 it suffices to introduce a stratification of $\overline{\mathrm{Q}}^{d}$ and to show that the *restriction of $\mathcal{W}_{E}^{d}$ to each stratum in $\overline{\mathbf{Q}}^{d}-Q^{d}$ appears in strictly negative cohomological degrees (with respect to the perverse $t$-structure). This stratification $\left\{Q^{\bar{\mu}}\right\}$ is introduced in Sect. 4.9. The description of the restriction of $\mathcal{W}_{E}^{d}$ to each stratum given in Proposition 4.12 will imply that it appears in strictly negative cohomological degrees (except for the open stratum).

4.8. Substacks of $\bar{Q}$ defined by orders of zeros of sections. The Langlands dual group to $G L_{n}$ is $G L_{n}\left(\overline{\mathbb{Q}}_{\ell}\right)$. In what follows we represent each weight of $G L_{n}\left(\overline{\mathbb{Q}}_{\ell}\right)$ as a string of integers $\left(d_{1}, \ldots, d_{n}\right)$, so that dominant weights satisfy $d_{1} \geq d_{2} \geq \ldots \geq d_{n}$. We denote the set of dominant weights by $P_{n}^{+}$. The irreducible finite-dimensional representation of $G L_{n}\left(\overline{\mathbb{Q}}_{\ell}\right)$ with highest weight $\mu \in P_{n}^{+}$ will be denoted by $V^{\mu}$. We denote by $w_{0}$ the longest element of the Weyl group of $G L_{n}$, which acts on the weights by the formula $w_{0} \cdot\left(d_{1}, \ldots, d_{n}\right)=\left(d_{n}, \ldots, d_{1}\right)$. For an anti-dominant weight $\mu$, we denote by $V_{\mu}$ the irreducible finite-dimensional representation of $G L_{n}$ with lowest weight $\mu$, i.e., $V_{\mu} \simeq V^{-w_{0}(\mu)}$.

Let $\bar{\mu}=\left\{\mu^{1}, \ldots, \mu^{m}\right\}$ be a collection of weights of $G L_{n}\left(\overline{\mathbb{Q}}_{\ell}\right)$, where some of the $\mu^{j}$ 's may coincide. We will denote by $X^{\bar{\mu}}$ the corresponding partially symmetrized power of $X$ with all the diagonals removed. In other words, if $m=m_{1}+\ldots+m_{s}$ is such that a given weight $\mu^{r}$ appears in the collection exactly $m_{r}$ times, then

$$
X^{\bar{\mu}}=X^{\left(m_{1}\right)} \times \ldots \times X^{\left(m_{s}\right)}-\Delta .
$$

We will think of a point $\bar{x}$ of $X^{\bar{\mu}}$ as a collection of pairwise distinct points $x^{j}$, $j=1, \ldots, m$, to each of which there is an assigned weight $\mu^{j}=\left(d_{1}^{j}, \ldots, d_{n}^{j}\right)$.

We associate to $\bar{\mu}$ a stack $\overline{\mathrm{Q}}^{\bar{\mu}}$, which classifies the data $\left(\mathcal{M},\left(s_{i}\right), \bar{x}\right)$, where $\mathcal{M}$ is a vector bundle of rank $n, \bar{x}$ is a point of $X^{\bar{\mu}}$ represented by a collection of distinct points $x^{j} \in X$, and

$$
s_{i}: \Omega^{(n-1)+\ldots+(n-i)}\left(\sum_{j}\left(d_{1}^{j}+\ldots+d_{i}^{j}\right) \cdot x^{j}\right) \hookrightarrow \Lambda^{i} \mathcal{M}
$$

are injective homomorphisms of coherent sheaves satisfying the Plücker relations from [FGV, BG].

The locus where all the maps $s_{i}$ are maximal embeddings (i.e., are bundle maps) is an open substack $2^{\bar{\mu}}$ of $\bar{Q}^{\bar{\mu}}$. In other words, $Q^{\bar{\mu}}$ classifies the data $\left(\mathcal{M},\left(\mathcal{M}_{i}\right),\left(\widetilde{s}_{i}\right), \bar{x}\right)$, where $\mathcal{M}$ is a rank $n$ bundle, $0=\mathcal{M}_{0} \subset \mathcal{M}_{1} \subset \ldots \subset \mathcal{M}_{n}=\mathcal{M}$ is a full flag of subbundles of $\mathcal{M}$, and $\widetilde{s}_{i}, i=1, \ldots, n$, is an isomorphism

$$
\Lambda^{i} \mathcal{M}_{i} \simeq \Omega^{(n-1)+\ldots+(n-i)}\left(\sum_{j}\left(d_{1}^{j}+\ldots+d_{i}^{j}\right) \cdot x^{j}\right) .
$$

For a fixed point $\bar{x} \in X^{\bar{\mu}}$, we will denote by $\overline{\mathrm{Q}}^{\bar{\mu}, \bar{x}}$ and $\mathrm{Q}^{\bar{\mu}, \bar{x}}$ the corresponding closed substacks of $\bar{Q}^{\bar{\mu}}$ and $Q^{\bar{\mu}}$, respectively.

For the reader's convenience, we identify the above stacks with those studied in FGV: $Q^{\bar{\mu}, \bar{x}} \simeq \operatorname{Bun}_{N}^{\mathcal{F}_{T}}$ and $\bar{Q}^{\bar{\mu}, \bar{x}} \simeq \overline{\operatorname{Bun}}_{N}^{\mathcal{F}_{T}}$, where $\mathcal{F}_{T}$ is the $T$-bundle on $X$, which corresponds to the $n$-tuple of line bundles

$$
\left(\Omega^{n-1}\left(\sum_{j} d_{1}^{j} \cdot x^{j}\right), \ldots, \Omega\left(\sum_{j} d_{n-1}^{j} \cdot x^{j}\right), \mathcal{O}\left(\sum_{j} d_{n}^{j} \cdot x^{j}\right)\right) .
$$


4.9. Stratification of $\overline{\mathcal{Q}}^{d}$. If the collection $\bar{\mu}$ satisfies the conditions

$$
d_{1}^{j}+\ldots+d_{i}^{j} \geq 0, \quad \forall i, j, \quad \sum_{i, j} d_{i}^{j}=d,
$$

then we have a natural closed embedding $\bar{Q}^{\bar{\mu}} \hookrightarrow \bar{Q}^{d}$, and so $Q^{\bar{\mu}}$ is a locally closed substack of $\overline{\mathcal{Q}}^{d}$. In particular, for a divisor $D=\sum_{j=1}^{m} d^{j} \cdot x^{j} \in X^{(d)}$ we have isomorphisms $\overline{\mathrm{Q}}^{D} \simeq \overline{\mathrm{Q}}^{\bar{\mu}, \bar{x}}$ and $\mathrm{Q}^{D} \simeq \mathrm{Q}^{\bar{\mu}, \bar{x}}$, where $\mu^{j}=\left(0, \ldots, d^{j}\right)$.

The following statement follows from [FGV], Corollary 2.2.9.

4.10. Lemma. The locally closed substacks $Q^{\bar{\mu}}$ with $\bar{\mu}$ satisfying the condition (4.9) form a stratification of $\overline{\mathrm{Q}}^{d}$.

4.11. Restrictions of $\mathcal{W}_{E}$ to the strata. The collection $\bar{\mu}$ is called anti-dominant if all the weights $\mu^{j}$ are anti-dominant. For an anti-dominant $\bar{\mu}$, we have a map $\mathrm{ev}^{\bar{\mu}}: Q^{\bar{\mu}} \rightarrow \mathbb{G}_{a}$ defined as in [FGV]. Namely,

$$
\begin{aligned}
\left.\mathrm{ev}: \mathrm{Q}^{\bar{\mu}} \rightarrow \prod_{i=1}^{n-1} \operatorname{Ext}^{1}\left(\mathcal{M}_{i} / \mathcal{M}_{i+1}, \mathcal{M}_{i-1} / \mathcal{M}_{i}\right)\right) & \\
& \rightarrow \prod_{i=1}^{n-1} H^{1}\left(X, \Omega\left(\sum_{j}\left(d_{i}^{j}-d_{i+1}^{j}\right) \cdot x^{j}\right)\right) \\
& \rightarrow H^{1}(X, \Omega)^{\oplus n-1} \simeq \mathbb{G}_{a}^{n-1} \stackrel{\operatorname{sum}}{\longrightarrow} \mathbb{G}_{a}
\end{aligned}
$$

(compare with formula (3.2)). We then set

$$
\stackrel{\circ}{\Psi}^{\bar{\mu}}:=\operatorname{ev}^{\bar{\mu} *}\left(\mathcal{J}_{\psi}\right) \otimes \overline{\mathbb{Q}}_{\ell}\left(\frac{\operatorname{dim}\left(Q^{\bar{\mu}}\right)}{2}\right)\left[\operatorname{dim}\left(Q^{\bar{\mu}}\right)\right] .
$$

Denote by $j^{\bar{\mu}}$ the embedding $Q^{\bar{\mu}} \rightarrow \bar{Q}^{\bar{\mu}}$. According to Theorem 2 of [FGV], the sheaf $\stackrel{\circ}{\Psi}^{\bar{\mu}}$ is clean, i.e., $\Psi^{\bar{\mu}}:=j_{!}^{\bar{\mu}}\left(\stackrel{\circ}{\Psi}^{\bar{\mu}}\right)$ is an irreducible perverse sheaf isomorphic to $j_{! *}^{\bar{\mu}}\left(\stackrel{\circ}{\Psi}^{\bar{\mu}}\right)$. In a similar way we define the perverse sheaves $\stackrel{\circ}{\Psi}^{\bar{\mu}, \bar{x}}$ and $\Psi^{\bar{\mu}, \bar{x}}$ on $Q^{\bar{\mu}, \bar{x}}$ and $\overline{\mathrm{Q}}^{\bar{\mu}}, \bar{x}$, respectively.

Next, to a local system $E$ on $X$ and an anti-dominant collection $\bar{\mu}$ we associate a local system $E_{\bar{\mu}}$ on $Q^{\bar{\mu}}$ as follows. Recall that we denote by $V_{\mu}$ the irreducible representation of $G L_{n}$ with lowest weight $\mu \in-P_{n}^{+}$. Let $E_{\mu}$ be the local system on $X$ associated to $E$ and $V_{\mu}$.

For $\bar{\mu}$ corresponding to the partition $m=m_{1}+\ldots+m_{s}$ consider the sheaf $\left(E_{\mu_{1}}\right)^{\left(m_{1}\right)} \otimes \ldots \otimes\left(E_{\mu_{s}}\right)^{\left(m_{s}\right)}$ on $X^{\left(m_{1}\right)} \times \ldots \times X^{\left(m_{s}\right)}$. Denote by $E_{\bar{\mu}}$ its restriction to the compliment of all diagonals, i.e., to $X^{\bar{\mu}}$. Let us denote by $\tau_{\bar{\mu}}$ the natural morphism from $Q^{\bar{\mu}}$ to $X^{\bar{\mu}}$ and set ${ }^{\prime} E_{\bar{\mu}}:=\tau_{\bar{\mu}}^{*}\left(E_{\bar{\mu}}\right)$.

Thus, ' $E_{\bar{\mu}}$ depends only on the positions of the points $x^{1}, \ldots, x^{m}$, and its fiber at $\left(\mathcal{M},\left(\widetilde{s}_{i}\right),\left(x^{1}, \ldots, x^{m}\right)\right)$ is $\bigotimes_{j=1}^{m} E_{\mu^{j}, x^{j}}$, where $E_{\mu^{j}, x^{j}}$ denotes the fiber of $E_{\mu^{j}}$ at $x^{j}$.

4.12. Proposition. The $*-$ restriction of $\mathcal{W}_{E}^{d}$ to $Q^{\bar{\mu}} \subset \bar{Q}^{d}$ is zero unless all weights $\mu^{j}$ are anti-dominant. When they are, this restriction is canonically identified with $\stackrel{\circ}{\Psi}^{\bar{\mu}} \otimes E_{\bar{\mu}} \otimes \overline{\mathbb{Q}}_{\ell}\left(\frac{d-m}{2}\right)[d-m]$. 
As was explained earlier, this proposition implies Proposition 4.6 Indeed, we had to show that $*$-restriction of $\mathcal{W}_{E}^{d}$ to each stratum in $Q^{\bar{\mu}} \subset \overline{\mathbf{Q}}^{d}-Q^{d}$ appears in negative cohomological degrees. According to Proposition 4.12 this restriction lives in the cohomological degree $-(d-m)$. However, since each $\mu^{j}=\left(d_{1}^{j}, \ldots, d_{n}^{j}\right)$ satisfies $d_{1}^{j} \leq \ldots \leq d_{n}^{j}$ and $d_{1}^{j}+\ldots+d_{n}^{j} \geq 0$, we obtain that $d-m \geq 0$ and the equality takes place only when every $\mu^{j}$ is of the form $(0, \ldots, 0,1)$. However, the stratum corresponding to this $\bar{\mu}$ is contained in $Q$.

The proof of Proposition 4.12 is given in the Appendix. A similar result is proved in Ly2.

4.13. The Whittaker function. In this subsection we will assume that $\mathbb{k}$ is the finite field $\mathbb{F}_{q}$. We will show that the function associated with the Whittaker sheaf may be identified with the restriction of the Whittaker function.

First we briefly recall the definition of the Whittaker function (see [FGKV], Sect. 2, for more details). Consider the group $G L_{n}(\mathbb{A})$ over the ring of adèles of $F=\mathbb{F}_{q}(X)$, and let $N(\mathbb{A})$ be its upper unipotent subgroup. Denote by $u_{i, i+1}$ the $i$-th component of the image of $u \in N(\mathbb{A})$ in $N(\mathbb{A}) /[N(\mathbb{A}), N(\mathbb{A})]$ corresponding to the $(i, i+1)$ entry of $u$. Recall that we have fixed a non-trivial additive character $\psi: \mathbb{F}_{q} \rightarrow \overline{\mathbb{Q}}_{\ell}^{\times}$. We define the character $\Psi$ of $N(\mathbb{A})$ by the formuld 4

$$
\Psi\left(\left(u_{x}\right)_{x \in|X|}\right)=\prod_{i=1}^{n-1} \prod_{x \in|X|} \psi\left(\operatorname{Tr}_{\mathbb{k}_{x} / \mathbb{F}_{q}}\left(\operatorname{Res}_{x} u_{x, i, i+1}\right)\right) .
$$

It follows from the residue theorem that $\Psi(u)=1$ if $u \in N(F)$.

Now let $E$ be a rank $n$ local system on $X$. Then there exists a unique (up to a non-zero scalar multiple) function $W_{E}$ on $G L_{n}(\mathbb{A})$, which is right $G L_{n}(\mathcal{O})$ invariant, left $(N(\mathbb{A}), \Psi)$-equivariant, and is a Hecke eigenfunction associated to $E$. This function is called the Whittaker function corresponding to $E$. CasselmanShalika [CS] and Shintani [Shi] have given an explicit formula for $W_{E}$ (see, e.g., Theorem 2.1 of [FGKV] $)$. The left $(N(\mathbb{A}), \Psi)$-equivariance of $W_{E}$ implies that it is left $N(F)$-invariant, where $F=\mathbb{F}_{q}(X)$. Therefore we obtain a function on the double quotient $Q=N(F) \backslash G L_{n}(\mathbb{A}) / G L_{n}(\mathcal{O})$. We denote this function also by $W_{E}$.

In the same way as in the the proof of Lemma 2.1 from FGKV, we identify the set of $\mathbb{F}_{q}$-points of $Q^{\bar{\mu}, \bar{x}}$, where $\mu^{j}=\left(d_{1}^{j}, \ldots, d_{n}^{j}\right), j=1, \ldots, m$, with the projection onto $Q$ of the subset

$$
N(\mathbb{A}) \cdot\left(\operatorname{diag}\left(\pi_{x}^{d_{n}(x)}, \ldots, \pi_{x}^{d_{1}(x)}\right)\right)_{x \in|X|} \cdot G L_{n}(\mathcal{O}) \subset G L_{n}(\mathbb{A}),
$$

where $d_{i}(x)=d_{i}^{j}$, if $x=x_{j}$, and $d_{i}(x)=0$, otherwise. Thus, we may embed the set $\overline{\mathrm{Q}}^{d}\left(\mathbb{F}_{q}\right)$ of isomorphism classes of $\mathbb{F}_{q}$-points of $\overline{\mathrm{Q}}^{d}$ into $Q$ for all $d \geq 0$. Comparing Proposition 4.12 with the Casselman-Shalika-Shintani formula, we obtain:

4.14. Proposition. The function $\mathrm{f}_{q}\left(\mathcal{W}_{E}^{d}\right)$ on $\bar{Q}^{d}\left(\mathbb{F}_{q}\right)$ corresponding to the sheaf $\mathcal{W}_{E}^{d}$ equals the restriction of the Whittaker function $W_{E}$ to $\overline{\mathbf{Q}}^{d}\left(\mathbb{F}_{q}\right) \subset Q$.

Furthermore, the geometric construction of the sheaf $\mathrm{Aut}_{E}^{\prime}$ described in this section translates at the level of functions into the construction due to Shalika [Sha]

\footnotetext{
${ }^{4}$ For this formula to be well-defined, we should consider a twisted version $G L_{n}^{J}(\mathbb{A})$ of $G L_{n}(\mathbb{A})$ introduced in [FGKV], Sect. 2 ; then $u_{i, i+1}$ is naturally a differential.
} 
and Piatetskii-Shapiro [PS1] (see Sect. 2 of [FGKV] for a review of this construction).

\section{Cleanness in Laumon's construction}

Let $E$ be an irreducible rank $n$ local system for which Conjecture 2.3 holds. In this section we derive Theorem 3.7 for $E$, i.e., we prove that the complex $\mathcal{F}_{E, k}$ on $\mathcal{E}_{k}^{0}$ is clean with respect to $j_{k}: \mathcal{E}_{k}^{0} \hookrightarrow \mathcal{E}_{k}$.

We will begin by stating a well-known lemma, which will serve as one of the key ingredients in the proof. Consider the following situation: let $\mathcal{E}$ be a vector bundle over a scheme (or stack) $Y$. Let us denote by $\rho: \mathcal{E} \rightarrow Y$ the projection, by $i: Y \rightarrow \mathcal{E}$ the zero section, and by $\mathcal{E}^{0} \stackrel{j}{\hookrightarrow} \mathcal{E}$ the complement of the zero section. Assume that $\mathcal{K}$ is a complex on $\mathcal{E}^{0}$, equivariant with respect to the $\mathbb{G}_{m}$-action.

5.1. Lemma. The complex $\mathcal{K}$ is clean with respect to $j$ if and only if $(\rho \circ j) !(\mathcal{K})=0$.

To prove the lemma, it suffices to note that cleanness of $\mathcal{K}$ is equivalent to the statement that $i^{!} j_{!}(\mathcal{K})=0$. But for any $\mathbb{G}_{m}$-equivariant complex $\mathcal{K}^{\prime}$ on $\mathcal{E}$, we have: $i^{!}\left(\mathcal{K}^{\prime}\right) \simeq \rho_{!}\left(\mathcal{K}^{\prime}\right)$.

Our proof of Theorem 3.7 will proceed by induction on the length of torsion in $\mathcal{C}_{k}$. Let us first consider the case where there is no torsion at all, i.e., we will show that $j_{k !}\left(\mathcal{F}_{E, k}\right) \rightarrow j_{k *}\left(\mathcal{F}_{E, k}\right)$ is an isomorphism on the open set $\rho_{k}^{-1}\left(\mathcal{C}_{k} \cap \operatorname{Bun}_{k}\right) \subset \mathcal{C}_{k}$.

Recall that $\rho_{k}$ (resp., $\rho_{k}^{0}$ ) denotes the projection $\mathcal{E}_{k} \rightarrow \mathcal{C}_{k}$ (resp., $\mathcal{E}_{k}^{0} \rightarrow \mathcal{C}_{k}$ ). On $\rho_{k}^{-1}\left(\mathcal{C}_{k} \cap \operatorname{Bun}_{k}\right), \mathcal{E}_{k}^{0}$ is the complement of the zero section in $\mathcal{E}_{k}$ and $\mathcal{F}_{E, k}$ is $\mathbb{G}_{m}$-equivariant. Thus, by Lemma 5.1 we are reduced to showing that

$$
\rho_{k}^{0}\left(\mathcal{F}_{E, k}\right)||_{\mathfrak{e}_{k} \cap \mathrm{Bun}_{k}}=0 .
$$

By Lemma 4.4, with $n$ replaced by $k$, we obtain that up to a cohomological shift and Tate's twist

$$
\left.\left.\rho_{k !}^{0}\left(\mathcal{F}_{E, k}\right)\right|_{\mathfrak{e}_{k} \cap \mathrm{Bun}_{k}^{d}} \simeq \mathrm{H}_{k, E}^{d}\left(q !\left(\Psi^{0}\right)\right)\right|_{\mathfrak{C}_{k} \cap \mathrm{Bun}_{k}^{d}} .
$$

The definition of $\mathcal{C}_{k}$ in Sect. 3.4 implies that if $\mathcal{C}_{k} \cap \operatorname{Bun}_{k} \neq \emptyset$, then $d>n k(2 g-2)$. Thus, the Vanishing Conjecture 2.3 implies $\rho_{k}^{0} !\left(\mathcal{F}_{E, k}\right) \mid \mathfrak{e}_{k} \cap \mathrm{Bun}_{k}=0$.

5.2. Induction on the length of torsion. To set up the induction, we fix some notation. For an integer $\ell$, let us write $\mathcal{C}_{k, \leq \ell}$ for the open substack of $\mathcal{C}_{k}$ consisting of coherent sheaves whose torsion is of length $\leq \ell$. Set $\mathcal{C}_{k,<\ell}=\mathcal{C}_{k, \leq \ell-1}$ to be the open substack in $\mathcal{C}_{k, \leq \ell}$ that corresponds to the locus where the torsion is of length $<\ell$. Finally, let $\mathcal{C}_{k, \ell}$ be the closed substack of $\mathcal{C}_{k, \leq \ell}$ corresponding to coherent sheaves whose torsion is precisely of length $\ell$.

Set $\mathcal{E}_{k, \leq \ell}=\rho_{k}^{-1}\left(\mathfrak{C}_{k, \leq \ell}\right), \mathcal{E}_{k,<\ell}=\rho_{k}^{-1}\left(\mathfrak{C}_{k,<\ell}\right)$, and $\mathcal{E}_{k, \ell}=\rho_{k}^{-1}\left(\mathfrak{C}_{k, \ell}\right)$. Furthermore, let us write

$$
\begin{aligned}
& \mathcal{E}_{k, \leq \ell}^{0}=\mathcal{E}_{k}^{0} \cap \mathcal{E}_{k, \leq \ell}, \quad \mathcal{E}_{k,<\ell}^{0}=\mathcal{E}_{k}^{0} \cap \mathcal{E}_{k,<\ell}, \\
& \mathcal{E}_{k}^{t}=\mathcal{E}_{k}-\mathcal{E}_{k}^{0}, \quad \mathcal{E}_{k, \ell}^{t}=\mathcal{E}_{k}^{t} \cap \mathcal{E}_{k, \ell}, \text { etc. }
\end{aligned}
$$

We assume, by induction, that $\mathcal{F}_{E, k}$ is clean with respect to the inclusion $\mathcal{E}_{k,<\ell}^{0} \hookrightarrow$ $\mathcal{E}_{k,<\ell}$. To show cleanness of $\mathcal{F}_{E, k}$ with respect to the inclusion $\mathcal{E}_{k, \leq \ell}^{0} \hookrightarrow \mathcal{E}_{k, \leq \ell}$, it suffices to prove cleanness of $\mathcal{F}_{E, k}$ with respect to $\left(\mathcal{E}_{k, \leq \ell}-\mathcal{E}_{k, \ell}^{t}\right) \hookrightarrow \mathcal{E}_{k, \leq \ell}$. We would like to argue in the same manner as we did above in the case when $\ell$ was zero and using Lemma 5.1. Unfortunately, we cannot apply this lemma directly, 
because $\mathcal{E}_{k, \leq \ell}$ is not a vector bundle over $\mathcal{E}_{k, \ell}^{t}$. However, it will become a vector bundle after a smooth base change.

Consider the stack $\operatorname{Coh}_{k, \leq \ell}$ which classifies coherent sheaves of generic rank $k$ with torsion of length $\leq \ell$, and the stack $\widetilde{\operatorname{Coh}}_{k, \leq \ell}$, which classifies the following data: $\mathcal{M}_{0} \in \mathrm{Bun}_{k}, \mathcal{T} \in \mathcal{C} h_{0}^{\ell}$, and a short exact sequence

$$
0 \rightarrow \mathcal{M}_{0} \rightarrow \mathcal{M} \rightarrow \mathcal{T} \rightarrow 0
$$

There is a canonical morphism $r: \widetilde{\operatorname{Coh}}_{k, \leq \ell} \rightarrow \operatorname{Coh}_{k, \leq \ell}$ which associates to a triple as above the coherent sheaf $\mathcal{N} \in \operatorname{Coh}_{k}$.

5.3. Lemma. The morphism $r$ is smooth.

Proof. First, the stack $\operatorname{Coh}_{k}$ is known to be smooth. One proves this simultaneously with the fact that $\operatorname{Coh}_{k}$ is indeed an algebraic stack in the smooth topology by covering it by a Hilbert scheme.

Therefore both stacks $\operatorname{Coh}_{k, \leq \ell}$ and $\widetilde{\operatorname{Coh}}_{k, \leq \ell}$ are smooth, the former being an open substack in $\operatorname{Coh}_{k}$, and the latter being a vector bundle over $\mathrm{Bun}_{k}$.

Hence in order to show that $r$ is smooth, it suffices to show that the fiber of

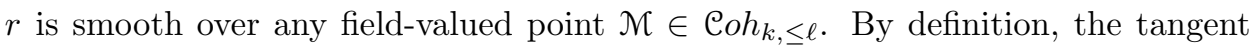
space to the fiber of $r$ at the point $0 \rightarrow \mathcal{M}_{0} \rightarrow \mathcal{M} \rightarrow \mathcal{T} \rightarrow 0$ is $\operatorname{Hom}\left(\mathcal{M}_{0}, \mathcal{T}\right)$. The dimension of $\operatorname{Hom}\left(\mathcal{M}_{0}, \mathcal{T}\right)$ is $k \cdot \ell$ because $\mathcal{M}_{0}$ is torsion-free. As $r$ is separable and the dimensions of the tangent spaces to the fibers are constant, we conclude that $r$ is smooth.

Since $r$ is smooth by Lemma 5.3 , it is sufficient to prove cleanness after this base change $r: \widetilde{\operatorname{Coh}}_{k, \leq \ell} \rightarrow \operatorname{Coh}_{k, \leq \ell}$. Consider the stack $\widetilde{\operatorname{Coh}}_{k, \leq \ell} \underset{\operatorname{Coh}_{k, \leq \ell}}{\times} \mathcal{E}_{k, \leq \ell}$. It classifies the following data:

$$
\begin{aligned}
& \mathcal{M} \in \mathcal{C}_{k}, \quad \mathcal{T} \in \operatorname{Coh}_{0}^{\ell}, \quad \mathcal{M}_{t f} \in \operatorname{Bun}_{k}, \\
& 0 \rightarrow \mathcal{M}_{t f} \rightarrow \mathcal{M} \rightarrow \mathcal{T} \rightarrow 0, \quad \Omega^{k-1} \stackrel{s}{\rightarrow} \mathcal{M} .
\end{aligned}
$$

Its substack

$$
\tilde{\mathcal{E}}_{k, \ell}^{t}=\widetilde{\operatorname{Coh}}_{k, \leq \ell} \underset{\operatorname{Coh}_{k, \leq \ell}}{\times} \mathcal{E}_{k, \ell}^{t}
$$

consists of the data (5.1) such that the extension is split and the image of $s$ belongs to the torsion part of $\mathcal{M}$. In other words, $\tilde{\mathcal{E}}_{k, \ell}^{t}$ classifies the data

$$
\mathcal{M}_{t f} \in \operatorname{Bun}_{k}, \quad \mathcal{T} \in \operatorname{Coh}_{0}^{\ell}\left(\text { such that } \mathcal{M}_{t f} \oplus \mathcal{T} \in \mathcal{C}_{k}\right), \quad \Omega^{k-1} \stackrel{\kappa}{\rightarrow} \mathcal{T} .
$$

Denote by $\tilde{\mathcal{E}}_{k, \leq \ell}$ the open substack of $\widetilde{\operatorname{Coh}}_{k, \leq \ell} \underset{\operatorname{Coh}_{k, \leq \ell}}{\times} \mathcal{E}_{k, \leq \ell}$ defined by the condition

$$
\operatorname{Hom}\left(\mathcal{M}_{t f}, \mathcal{L}^{\text {est }}\right)=0 ;
$$

here $\mathcal{L}^{\text {est }}$ is the line bundle of Sect. 3.2 The above condition guarantees that $\mathcal{M}_{t f} \oplus \mathcal{T} \in \mathcal{C}_{k}$. Set

$$
\tilde{\varepsilon}_{k, \leq \ell}^{0}=\tilde{\mathcal{E}}_{k, \leq \ell} \cap\left(\widetilde{\operatorname{Coh}}_{k, \leq \ell} \underset{\operatorname{eoh} k, \leq \ell}{\times} \mathcal{E}_{k, \leq \ell}^{0}\right) .
$$

Obviously, $\tilde{\mathcal{E}}_{k, \ell}^{t}$ is contained in $\tilde{\mathcal{E}}_{k, \leq \ell}$. 
There is a natural morphism $\rho_{k, \ell}: \tilde{\mathcal{E}}_{k, \leq \ell} \rightarrow \tilde{\mathcal{E}}_{k, \ell}^{t}$ which maps the quadruple in the definition of $\tilde{\mathcal{E}}_{k, \leq \ell}$ to the data $\left(\mathcal{M}_{t f}, \mathcal{T}, \kappa\right)$, where $\kappa$ is the composition $\Omega^{k-1} \stackrel{s}{\rightarrow} \mathcal{M} \rightarrow$ $\mathcal{T}$. In this way $\tilde{\tilde{\varepsilon}}_{k, \leq \ell}$ becomes a vector bundle over $\tilde{\mathcal{E}}_{k, \ell}^{t}$; the fiber over $\left(\mathcal{M}_{t f}, \mathcal{T}, \kappa\right)$ can be canonically identified with the vector space

$$
\operatorname{Ext}^{1}\left(\operatorname{Cone}\left(\Omega^{k-1} \stackrel{\kappa}{\longrightarrow} \mathcal{T}\right), \mathcal{M}_{t f}\right) \text {. }
$$

Let $\rho_{k, \ell}^{0}$ denote the composition $\tilde{\mathcal{E}}_{k, \leq \ell}^{0} \hookrightarrow \tilde{\mathcal{E}}_{k, \leq \ell} \stackrel{\rho_{k, \ell}}{\longrightarrow} \tilde{\mathcal{E}}_{k, \ell}^{t}$.

Let $\widetilde{\mathcal{F}}_{E, k}$ denote the pull-back of $\mathcal{F}_{E, k}$ to $\tilde{\mathcal{E}}_{k, \leq \ell}^{0}$. One readily verifies that $\widetilde{\mathcal{F}}_{E, k}$ is equivariant with respect to the $\mathbb{G}_{m}$-action along the fibers of $\rho_{k, \ell}: \tilde{\mathcal{E}}_{k, \leq \ell} \rightarrow \tilde{\mathcal{E}}_{k, \ell}^{t}$.

The assertion of the theorem reduces to the fact that $\widetilde{\mathcal{F}}_{E, k}$ is clean with respect to the open embedding $\tilde{\mathcal{E}}_{k, \leq \ell}^{0} \hookrightarrow \tilde{\mathcal{E}}_{k, \leq \ell}$ and we already know this assertion on the open substack $\tilde{\mathcal{E}}_{k, \leq \ell}-\tilde{\mathcal{E}}_{k, \ell}^{t}$.

By applying Lemma 5.1 to $\widetilde{\mathcal{F}}_{E, k}$ extended by 0 from $\tilde{\mathcal{E}}_{k, \leq \ell}^{0}$ to $\tilde{\mathcal{E}}_{k, \leq \ell}-\tilde{\mathcal{E}}_{k, \ell}^{t}$, we reduce our assertion to showing that

$$
\rho_{k, \ell !}^{0}\left(\widetilde{\mathcal{F}}_{E, k}\right)=0 .
$$

5.4. Proof of formula (5.3). Recall the stack $\widetilde{Q}$ of Sect. 3.1 and let us denote by $\widetilde{Q}_{k}$ (resp., $\mathcal{Q}_{k}, \mathcal{Q}_{k}^{0}, \overline{\mathcal{Q}}_{k}$ ) its version with $n$ replaced by $k$. In particular, $\mathcal{Q}_{k}^{0}$ classifies points of the form $\left(\mathcal{M}^{0}, \beta,\left(\mathcal{M}_{i}^{0}\right),\left(\widetilde{s}_{i}\right)\right)$, where the map $\beta: \mathcal{M}^{0} \rightarrow \mathcal{M}^{0}=\mathcal{M}_{n}^{0}$ is the identity. We will denote such a point simply by $\left(\mathcal{M}^{0},\left(\widetilde{s}_{i}\right)\right)$. Denote by $\widetilde{\mathcal{W}}_{E, k}$ the perverse sheaf on $\widetilde{Q}_{k}$ defined as in Sect. 3.1.

Consider the Cartesian product $\widetilde{\mathcal{Q}}_{k} \underset{\mathrm{Coh}_{k}^{\prime}}{\times} \tilde{\mathcal{E}}_{k, \leq \ell}^{0}$. This is the stack that classifies the data of

$$
\begin{aligned}
& \mathcal{M}_{t f} \in \operatorname{Bun}_{k} \text { with } \operatorname{Hom}\left(\mathcal{M}_{t f}, \mathcal{L}^{\text {est }}\right)=0, \quad \mathcal{T} \in \mathcal{C o h}_{0}^{\ell}, \\
& 0 \rightarrow \mathcal{M}_{t f} \rightarrow \mathcal{M} \rightarrow \mathcal{T} \rightarrow 0, \quad \mathcal{M}^{0} \hookrightarrow \mathcal{M}, \\
& \left(\mathcal{M}^{0}, \widetilde{s}_{1}, \ldots, \widetilde{s}_{k}\right) \in \mathcal{Q}_{k}^{0} .
\end{aligned}
$$

By Lemma 3.5 $\widetilde{\mathcal{F}}_{E, k}$ is the direct image (with compact supports) under the map $\widetilde{Q}_{k} \underset{\operatorname{Coh} h_{k}^{\prime}}{\times} \tilde{\mathcal{E}}_{k, \leq \ell}^{0} \rightarrow \tilde{\mathcal{E}}_{k, \leq \ell}^{0}$ of the pull-back of $\widetilde{\mathcal{W}}_{E, k}$ from $\widetilde{Q}_{k}$ to $\widetilde{\mathcal{Q}}_{k} \underset{\operatorname{Coh}_{k}^{\prime}}{\times} \tilde{\mathcal{E}}_{k, \leq \ell}^{0}$. Hence, in order to prove (5.3), it suffices to show that the compactly supported cohomology of the fiber of $\widetilde{Q}_{k} \underset{\operatorname{Coh}_{k}^{\prime}}{\times} \tilde{\mathcal{E}}_{k, \leq \ell}^{0} \rightarrow \tilde{\mathcal{E}}_{k, \leq \ell}^{0} \rightarrow \tilde{\mathcal{E}}_{k, \ell}^{t}$ with coefficients in the pull-back of $\widetilde{\mathcal{W}}_{E, k}$ vanishes. To this end, let us fix a point $\left(\mathcal{M}_{t f} \in \operatorname{Bun}_{k}, \mathcal{T} \in \operatorname{Coh}_{0}^{\ell}, \kappa: \Omega^{k-1} \rightarrow \mathcal{T}\right)$ in $\tilde{\varepsilon}_{k, \ell}^{t}$ and analyze the fiber over this point. Let us write $Y$ for the closed substack of the the fiber which lies over a fixed point $\left(\mathcal{M}^{0}, \widetilde{s}_{1}, \ldots, \widetilde{s}_{k}\right) \in Q_{k}^{0}$ and where the composition $\phi: \mathcal{M}^{0} \rightarrow \mathcal{M} \rightarrow \mathcal{T}$ is also fixed.

From the discussion above we conclude that (5.3) follows if we show that for all

$$
\left(\mathcal{M}_{t f} \in \operatorname{Bun}_{k}, \quad \mathcal{T} \in \operatorname{Coh}_{0}^{\ell}, \quad\left(\mathcal{M}^{0}, \widetilde{s}_{1}, \ldots, \widetilde{s}_{k}\right) \in \mathcal{Q}_{k}^{0}, \quad \phi: \mathcal{M}^{0} \rightarrow \mathcal{T}\right)
$$

as above, we have

$$
H_{c}^{\bullet}\left(Y,\left.\widetilde{\mathcal{W}}_{E, k}\right|_{Y}\right)=0
$$

To prove this, we will first reduce to the case when $\phi: \mathcal{M}^{0} \rightarrow \mathcal{T}$ is surjective. Let us denote by $\mathcal{T}^{\prime}$ the image of $\phi$ and by $\mathcal{T}^{\prime \prime}$ the cokernel of $\kappa$; write $\ell^{\prime}$ (resp., $\ell^{\prime \prime}$ ) 
for the length of $\mathcal{T}^{\prime}$ (resp., $\left.\mathcal{T}^{\prime \prime}\right)$. Let $Y^{\prime}$ be the scheme defined in the same way as $Y$, but for

$$
\left(\mathcal{M}_{t f}, \quad \mathcal{T}^{\prime}, \quad\left(\mathcal{M}^{0}, \widetilde{s}_{1}, \ldots, \widetilde{s}_{k}\right) \in Q_{k}^{0}, \quad \phi^{\prime}: \mathcal{M}^{0} \rightarrow \mathcal{T}^{\prime}\right)
$$

We have a natural map v $: Y \rightarrow Y^{\prime}$, which associates to a point

$$
\left(0 \rightarrow \mathcal{M}_{t f} \rightarrow \mathcal{M} \rightarrow \mathcal{T} \rightarrow 0, \mathcal{M}^{0} \hookrightarrow \mathcal{M}\right)
$$

the point

$$
\left(0 \rightarrow \mathcal{M}_{t f} \rightarrow \mathcal{M}^{\prime} \rightarrow \mathcal{T}^{\prime} \rightarrow 0, \phi^{\prime}: \mathcal{M}^{0} \hookrightarrow \mathcal{M}^{\prime}\right),
$$

where $\mathcal{M}^{\prime}$ is the preimage of $\mathcal{T}^{\prime}$ under $\mathcal{M} \rightarrow \mathcal{T}$.

5.5. Lemma. The complexes $\mathrm{v} !\left(\left.\widetilde{\mathcal{W}}_{E, k}\right|_{Y}\right)$ and $\left.\widetilde{\mathcal{W}}_{E, k}\right|_{Y^{\prime}} \otimes\left(\mathcal{L}_{E}^{\ell^{\prime \prime}}\right)_{\mathcal{T}^{\prime \prime}}$ are isomorphic up to a cohomological shift and Tate's twist; here $\left(\mathcal{L}_{E}^{\ell^{\prime \prime}}\right)_{\mathcal{T}^{\prime \prime}}$ is the stalk of Laumon's sheaf at $\mathcal{T}^{\prime \prime} \in \operatorname{Coh}_{0}^{\ell^{\prime \prime}}$.

Proof. Let us recall the following basic property of Laumon's sheaf $\mathcal{L}_{E}$ (cf. [Lau2]):

Consider the stack $\mathrm{Fl}_{0}^{d^{\prime}, d^{\prime \prime}}$ that classifies short exact sequences

$$
0 \rightarrow \tilde{\mathfrak{T}}^{\prime} \rightarrow \tilde{\mathfrak{T}} \rightarrow \tilde{\mathfrak{T}}^{\prime \prime} \rightarrow 0, \quad \tilde{\mathfrak{T}}^{\prime} \in \operatorname{Coh}_{0}^{d^{\prime}}, \quad \tilde{\mathfrak{T}}^{\prime \prime} \in \operatorname{Coh}_{0}^{d^{\prime \prime}}
$$

Let $\mathfrak{p}$ denote the natural projection $\mathrm{Fl}_{0}^{\ell^{\prime}, \ell^{\prime \prime}} \rightarrow \operatorname{Coh}_{0}^{d}\left(\right.$ here $\left.d=d^{\prime}+d^{\prime \prime}\right)$ that associates to a short exact sequence as above its middle term, and let $\mathfrak{q}: \mathrm{Fl}_{0}^{d^{\prime}, d^{\prime \prime}} \rightarrow \operatorname{Coh}_{0}^{d^{\prime}} \times$ Coh ${ }_{0}^{d^{\prime \prime}}$ denote the other natural projection.

In Lau2 Laumon proved that

$$
\mathfrak{q} ! \circ \mathfrak{p}^{*}\left(\mathcal{L}_{E}^{d}\right) \simeq \mathcal{L}_{E}^{d^{\prime}} \otimes \mathcal{L}_{E}^{d^{\prime \prime}}
$$

We have a natural map $Y^{\prime} \rightarrow \operatorname{Coh}_{0}^{d^{\prime}} \times \operatorname{Coh}_{0}^{d^{\prime \prime}}$ that sends the data of

$$
\left(0 \rightarrow \mathcal{M}_{t f} \rightarrow \mathcal{M}^{\prime} \rightarrow \mathcal{T}^{\prime} \rightarrow 0, \quad \mathcal{M}^{0} \hookrightarrow \mathcal{M}^{\prime}\right)
$$

to $\left(\mathcal{M}^{\prime} / \mathcal{M}^{0}, \mathcal{T}^{\prime \prime}\right)$ with $d^{\prime}=\operatorname{deg}\left(\mathcal{M}^{\prime}\right)-\operatorname{deg}\left(\mathcal{M}^{0}\right), d^{\prime \prime}=\ell^{\prime \prime}$ and a map $Y \rightarrow \mathrm{Fl}_{0}^{d^{\prime}, d^{\prime \prime}}$ that sends

$$
\left(0 \rightarrow \mathcal{M}_{t f} \rightarrow \mathcal{M} \rightarrow \mathcal{T} \rightarrow 0, \quad \mathcal{M}^{0} \hookrightarrow \mathcal{M}\right)
$$

to

$$
0 \rightarrow \mathcal{M}^{\prime} / \mathcal{M}^{0} \rightarrow \mathcal{M} / \mathcal{M}^{0} \rightarrow \mathcal{T}^{\prime \prime} \rightarrow 0 .
$$

Note that since $\mathcal{M}^{0}$ is fixed, $\left.\widetilde{\mathcal{W}}_{E, k}\right|_{Y}$ is isomorphic to the pull-back of $\mathfrak{p}^{*}\left(\mathcal{L}_{E}^{d}\right)$ under this map. Similarly, $\left.\widetilde{\mathcal{W}}_{E, k}\right|_{Y^{\prime}} \otimes\left(\mathcal{L}_{E}^{\ell^{\prime \prime}}\right)_{\mathcal{T}^{\prime \prime}}$ is isomorphic to the pull-back of $\mathcal{L}_{E}^{d^{\prime}} \otimes \mathcal{L}_{E}^{d^{\prime \prime}}$ under $Y^{\prime} \rightarrow \operatorname{Coh}_{0}^{d^{\prime}} \times \operatorname{Coh}_{0}^{d^{\prime \prime}}$.

We have the following diagram:

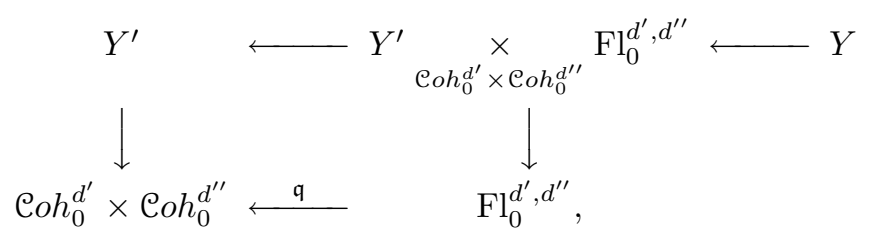

in which the composed upper horizontal map is v. Moreover, it is easy to see that the map $Y^{\prime} \underset{\operatorname{Coh}_{0}^{d^{\prime}} \times \operatorname{Coh}_{0}^{d^{\prime \prime}}}{\times} \mathrm{Fl}_{0}^{d^{\prime}, d^{\prime \prime}} \leftarrow Y$ is a fibration with fibers being affine spaces 
of the same dimension. Therefore, up to Tate's twist and a cohomological shift, $\mathrm{v} !\left(\left.\widetilde{\mathcal{W}}_{E, k}\right|_{Y}\right)$ is isomorphic to the pull-back under $Y^{\prime} \rightarrow \operatorname{Coh}_{0}^{d^{\prime}} \times \operatorname{Coh}_{0}^{d^{\prime \prime}}$ of $\mathfrak{q}_{!} \circ \mathfrak{p}^{*}\left(\mathcal{L}_{E}^{d}\right)$.

Hence, the assertion of the lemma follows from (5.8).

5.6. End of the proof of formula (5.3). The above considerations show that it suffices to treat the case when $\phi: \mathcal{M}^{0} \rightarrow \mathcal{T}$ is surjective. Let $\mathcal{M}^{1}$ denote the kernel of $\phi$. Let us observe that the scheme $Y$ can be identified with the scheme $\operatorname{Hom}^{0}\left(\mathcal{M}^{1}, \mathcal{M}_{t f}\right)$ of injective maps $\mathcal{M}^{1} \rightarrow \mathcal{M}_{t f}$

Indeed, to

$$
0 \rightarrow \mathcal{M}_{t f} \rightarrow \mathcal{M} \rightarrow \mathcal{T} \rightarrow 0, \quad \mathcal{M}^{0} \hookrightarrow \mathcal{M}
$$

we associate $\mathcal{M}^{1} \hookrightarrow \mathcal{M}^{0} \hookrightarrow \mathcal{M}$, which maps into $\mathcal{M}_{t f}$ by assumption. And vice versa: to an embedding $\mathcal{M}^{1} \rightarrow \mathcal{M}_{t f}$ we associate $\mathcal{M}=\mathcal{M}_{t f} \underset{\mathcal{N}^{1}}{\oplus} \mathcal{M}^{0}$.

Moreover, the sheaf $\left.\widetilde{\mathcal{W}}_{E, k}\right|_{Y}$ becomes isomorphic to $\pi^{*}\left(\mathcal{L}_{E}^{d}\right)$, where $d=\operatorname{deg}\left(\mathcal{M}_{t f}\right)$ $-\operatorname{deg}\left(\mathcal{M}^{1}\right)$. Therefore, the cohomology $H_{c}\left(Y,\left.\widetilde{\mathcal{W}}_{E, k}\right|_{Y}\right)$ equals the cohomology appearing in Conjecture 2.5.

By assumption, the vector bundle $\mathcal{M}_{t f}$ satisfies: $\operatorname{Hom}\left(\mathcal{M}_{t f}, \mathcal{L}^{\text {est }}\right)=0$. Hence, by the condition on $\mathcal{L}^{\text {est }}$ (cf. Sect. 3.2), $\operatorname{deg}\left(\mathcal{M}_{t f}\right)>n k(2 g-2)$, and so $d=\operatorname{deg}\left(\mathcal{M}_{t f}\right)+$ $\ell>n k(2 g-2)$.

The required vanishing statement now follows from Conjecture 2.5 applied to $E$. This completes the proof of formula (5.3) and Theorem 3.7 .

\section{Descent of the Sheaf $\mathcal{F}_{E, n}$}

As in the previous section, we keep the assumption that the local system $E$ is irreducible and that Conjecture 2.3 holds for $E$. Our goal here is to prove Theorem 3.9 .

Having established Theorem 3.7 for all $k=1, \ldots, n-1$ we know, according to Corollary 3.8 , that over $\mathcal{C}_{n}^{d}$ the complex $\mathcal{F}_{E, n} \otimes \overline{\mathbb{Q}}_{\ell}\left(\frac{d}{2}\right)[d]$ is an irreducible perverse sheaf.

6.1. Euler characteristics. The morphism $\rho_{n}^{0}: \mathcal{E}_{n}^{0} \rightarrow \mathcal{C}_{n}$ is smooth of relative dimension $d-n^{2}(g-1)$, and the sheaf $\left.\mathcal{F}_{E, n} \otimes \overline{\mathbb{Q}}_{\ell}\left(\frac{d}{2}\right)[d]\right|_{\mathcal{E}_{n}^{0} \cap\left(\rho_{n}^{0}\right)^{-1}\left(\mathcal{C}_{n}^{d}\right)}$ is perverse and irreducible. Hence in order to prove Theorem 3.9 it suffices to show that when $d \geq c_{g, n}$, the restriction $\left.\mathcal{F}_{E, n}\right|_{\left(\rho_{n}^{0}\right)^{-1}\left(\mathcal{C}_{n} \cap \mathrm{Bun}_{n}^{d}\right)}$ is non-zero and that it descends to a perverse sheaf on $\mathcal{C}_{n} \cap \operatorname{Bun}_{n}^{d}$.

Recall from Lemma 4.4 that

$$
\left.\left.\mathcal{F}_{E, n}\right|_{\left(\rho_{n}^{0}\right)^{-1}\left(\mathcal{C}_{n} \cap \mathrm{Bun}_{n}^{d}\right)} \simeq \operatorname{Aut}_{E}^{\prime}\right|_{\left(\rho_{n}^{0}\right)^{-1}\left(\mathcal{C}_{n} \cap \operatorname{Bun}_{n}^{d}\right)},
$$

up to a cohomological shift.

The proof will be based on the following proposition:

6.2. Proposition. The Euler characteristics of the stalks of $\left.\mathrm{Aut}_{E}^{\prime}\right|_{\mathrm{Bun}_{n}^{\prime}}$ are constant along the fibers of the projection $\varrho_{n}: \operatorname{Bun}_{n}^{\prime} \rightarrow \operatorname{Bun}_{n}$. Moreover, they are not identically equal to zero over $\left(\varrho_{n}\right)^{-1}\left(\varrho_{n} \cap \operatorname{Bun}_{n}^{d}\right)\left(\right.$ for $\left.d \geq c_{g, n}\right)$. 
6.3. Derivation of descent from Proposition 6.2. Suppose $d \geq c_{g, n}$. Then the perverse sheaf $\left.\mathcal{F}_{E, n}\right|_{\mathcal{E}_{n}^{0} \cap\left(\rho_{n}^{0}\right)^{-1}\left(\mathfrak{C}_{n}^{d}\right)} \otimes \overline{\mathbb{Q}}_{\ell}\left(\frac{d}{2}\right)[d]$ is the Goresky-MacPherson extension of a local system on a locally closed substack $U^{\prime}$ of $\mathcal{E}_{n}^{0}$, contained inside the open substack $\left(\rho_{n}^{0}\right)^{-1}\left(\mathcal{C}_{n} \cap \operatorname{Bun}_{n}^{d}\right) \subset \mathcal{E}_{n}^{0}$.

There exists a smooth locally closed substack $U_{1} \subset \mathcal{C}_{n} \cap \operatorname{Bun}_{n}^{d}$, such that if we set $U_{1}^{\prime}=\left(\rho_{n}^{0}\right)^{-1}\left(U_{1}\right)$, the intersection $U_{1}^{\prime} \cap U^{\prime}$ is open and dense in $U^{\prime}$ and $\rho_{n}^{0}: U_{1}^{\prime} \cap U^{\prime} \rightarrow U_{1}$ is surjective. Since $\rho_{n}^{0}$ is smooth, in order to prove Theorem 3.9 it suffices to show that $\left.\mathcal{F}_{E, n} \otimes \overline{\mathbb{Q}}_{\ell}\left(\frac{d}{2}\right)[d]\right|_{U_{1}^{\prime}}$ is a pull-back of a local system on $U_{1}$.

We have the following general result:

6.4. Lemma. Let $Y$ be a smooth scheme (or stack) and let $\mathcal{K}$ be an irreducible perverse sheaf on $Y$. If the Euler characteristics of the stalks of $\mathcal{K}$ are the same at all $\overline{\mathbb{k}}$-points of $Y$, then $\mathcal{K}$ is a local system. If these Euler characteristics are not identically equal to 0 , then $\mathcal{K} \neq 0$.

Proof. Let $Y_{0} \subset Y$ be the maximal open subset over which $\mathcal{K}$ is a local system. By the irreducibility assumption, $\mathcal{K}$ is the Goresky-MacPherson extension of a local system on $Y_{0}$. Since $Y$ is smooth, it is enough to show that $Y-Y_{0}$ is of codimension $\geq 2$.

Suppose this is not so. Then $Y-Y_{0}$ contains a divisor. Let $A$ denote the strict Henselization of the local ring at the generic point of this divisor and let $\eta$ (resp., $s$ ) be the generic (resp., closed) point of $\operatorname{Spec}(A)$.

By our assumptions, $\left.\mathcal{K}\right|_{\operatorname{Spec}(A)}$ is the Goresky-MacPherson extension of a local system on $\eta$. The stalk $\mathcal{K}_{\eta}$ is a representation of the Galois group $\Gamma$ of the field of fractions of $A$, and we have $\mathcal{K}_{s} \simeq\left(\mathcal{K}_{\eta}\right)^{\Gamma}$.

By the assumption on the Euler characteristics, $\operatorname{dim}\left(\mathcal{K}_{s}\right)=\operatorname{dim}\left(\mathcal{K}_{\eta}\right)$, i.e., the representation of $\Gamma$ on $\mathcal{K}_{\eta}$ is trivial. But this means that $\mathcal{K}$ extends as a local system to the entire $\operatorname{Spec}(A)$, which is a contradiction.

Let us show that this lemma is applicable for $Y=U_{1}^{\prime}$ and $\mathcal{K}=\left.\mathcal{F}_{E, n} \otimes \overline{\mathbb{Q}}_{\ell}\left(\frac{d}{2}\right)[d]\right|_{U_{1}^{\prime}}$. Indeed, the Euler characteristics of stalks of $\left.\mathcal{F}_{E, n}\right|_{U_{1}^{\prime}}$ are constant along the fibers of $U_{1}^{\prime} \rightarrow U_{1}$, by Proposition 6.2. Moreover, they are constant on $U_{1}^{\prime} \cap U^{\prime}$, since $\mathcal{F}_{E, n}$ is a local system there. Hence, the Euler characteristics are constant on all of $U_{1}^{\prime}$, since $U_{1}^{\prime} \cap U^{\prime} \rightarrow U_{1}$ is surjective.

Thus, we obtain that $\left.\mathcal{F}_{E, n} \otimes \overline{\mathbb{Q}}_{\ell}\left(\frac{d}{2}\right)[d]\right|_{U_{1}^{\prime}}$ is a non-zero local system.

By definition, $U_{1}^{\prime}$ is a complement to the zero section in the vector bundle $\left.\varepsilon_{n}\right|_{U_{1}}$. By construction, $\mathcal{F}_{E, n}$ is equivariant with respect to the natural $\mathbb{G}_{m}$-action along the fibers of the projection $\rho_{n}^{0}: U_{1}^{\prime} \rightarrow U_{1}$. Therefore, the assertion of Theorem 3.9] follows from the next lemma applied to $\mathcal{E}:=\left.\mathcal{E}_{n}\right|_{U_{1}}, \mathcal{K}:=\left.\mathcal{F}_{E, n} \otimes \overline{\mathbb{Q}}_{\ell}\left(\frac{d}{2}\right)[d]\right|_{U_{1}^{\prime}}$.

6.5. Lemma. Let $\mathcal{E} \rightarrow Y$ be a vector bundle and let us denote by $\mathcal{E}^{0}$ the complement to the zero section. Let $\mathcal{K}$ be a local system on $\mathcal{E}^{0}$, equivariant with respect to the $\mathbb{G}_{m}$-action along the fibers. Then $\mathcal{K}$ descends to a local system on $Y$.

Proof. This follows from the fact that any local system on a projective space is isomorphic to the trivial local system.

Now we prove Proposition 6.2. The first step is the following statement.

6.6. Lemma. Let $E^{\prime}$ be another rank $n$ local system, not necessarily irreducible. Then the Euler characteristics of $\mathrm{Aut}_{E}^{\prime}$ and $\mathrm{Aut}_{E^{\prime}}^{\prime}$ are equal at any given $\overline{\mathbb{k}}$-point of $\operatorname{Bun}_{n}^{\prime}$. 
In order to prove the lemma, we will use the following corollary of a theorem of Deligne from [II], Corollary 2.10:

6.7. Theorem. Let $f: Y_{1} \rightarrow Y_{2}$ be a proper morphism of schemes (or a proper representable map of stacks). Let $\mathcal{K}$ and $\mathcal{K}^{\prime}$ be two complexes on $Y_{1}$, which are locally isomorphic, by which we mean that they can be represented as inverse limits of étale-locally isomorphic complexes with torsion coefficients. Then $f_{!}(\mathcal{K})$ and $f_{!}\left(\mathcal{K}^{\prime}\right)$ have equal Euler characteristics at every $\overline{\mathbb{k}}$-point of $Y_{2}$.

Recall the stack $\bar{Q}$ and note that the group $\left(\mathbb{G}_{m}\right)^{n}$ acts on it by the rule

$$
\left(c_{1}, \ldots, c_{n}\right) \cdot\left(\mathcal{M}, s_{1}, \ldots, s_{n}\right)=\left(\mathcal{M}, c_{1} \cdot s_{1}, \ldots, c_{n} \cdot s_{n}\right) .
$$

Consider the quotient $\bar{Q}_{r}:=\overline{\mathrm{Q}} /\left(\mathbb{G}_{m}\right)^{n-1}$, where $\left(\mathbb{G}_{m}\right)^{n-1} \subset\left(\mathbb{G}_{m}\right)^{n}$ corresponds to the omission of the first copy of $\mathbb{G}_{m}$. Then the morphism $\nu: \overline{\mathrm{Q}} \rightarrow \operatorname{Bun}_{n}^{\prime}$ factors as

$$
\overline{\mathrm{Q}} \rightarrow \overline{\mathrm{Q}}_{r} \stackrel{\nu_{r}}{\rightarrow} \operatorname{Bun}_{n}^{\prime}
$$

The following is proved in $[\mathrm{BG}$, Proposition 1.2.2:

6.8. Lemma. The morphism $q_{r}: \bar{Q} /\left(\mathbb{G}_{m}\right)^{n} \rightarrow \mathrm{Bun}_{n}$ is representable and proper.

We obtain from this lemma that $\nu_{r}: \bar{Q}_{r} \rightarrow \operatorname{Bun}_{n}^{\prime}$ is also proper.

Now let us take the quotient of the diagram (4.5) by $\left(\mathbb{G}_{m}\right)^{n-1}$ :

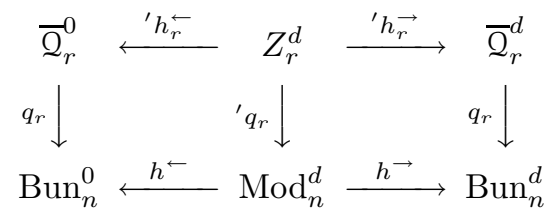

Denote by $\Psi_{r}^{0}$ the !-direct image of $\Psi^{0}$ under $\overline{\mathbf{Q}}^{0} \rightarrow \bar{Q}_{r}^{0}$. It is clear that Aut $_{E}^{\prime}$ can be written as

$$
\operatorname{Aut}_{E}^{\prime}=\nu_{r !}{ }^{\prime} h_{r} !\left({ }^{\prime} h_{r}^{\leftarrow *}\left(\Psi_{r}^{0}\right) \otimes{ }^{\prime} q_{r}^{*} \pi^{*}\left(\mathcal{L}_{E}^{d}\right)\right) \otimes \overline{\mathbb{Q}}_{\ell}\left(\frac{d \cdot n}{2}\right)[d \cdot n],
$$

and similarly for $E^{\prime}$.

This formula and Theorem 6.7 readily imply the equality of the Euler characteristics of $\mathrm{Aut}_{E}^{\prime}$ and $\mathrm{Aut}_{E^{\prime}}^{\prime}$. Indeed, the morphism $\nu_{r} \circ^{\prime} h_{r}: Z_{r}^{d} \rightarrow \mathrm{Bun}_{n}^{\prime}$ is proper and the complexes

$$
{ }^{\prime} h_{r}^{\leftarrow *}\left(\Psi_{r}^{0}\right) \otimes{ }^{\prime} q_{r}^{*} \pi^{*}\left(\mathcal{L}_{E}^{d}\right), \quad{ }^{\prime} h_{r}^{\leftarrow *}\left(\Psi_{r}^{0}\right) \otimes^{\prime} q_{r}^{*} \pi^{*}\left(\mathcal{L}_{E^{\prime}}^{d}\right)
$$

are locally isomorphic, since so are the corresponding Laumon's sheaves $\mathcal{L}_{E}^{d}$ and $\mathcal{L}_{E^{\prime}}^{d}$. This completes the proof of Lemma 6.6.

We remark that formula (6.1) is the generalization of the Radon transform construction (as opposed to the Fourier transform construction from Sect. 3.2) in Drinfeld's original proof $[\mathrm{Dr}]$ of the Langlands conjecture in the case of $G L_{2}$.

6.9. Conclusion of the proof of Proposition 6.2. According to Lemma 6.6, in order to prove Proposition 6.2 it suffices to show that there exists at least one local system $E$, for which the statement of Proposition 6.2 is true. Hence it suffices to prove it for the trivial local system. Using the reduction technique of [BBD], Sect. 6.1.7, we obtain: 
6.10. Lemma. Suppose that Proposition 6.2 holds when $E$ is the trivial local system in the case when the ground field $\mathbb{k}$ is a finite field of characteristic $p$. Then Proposition 6.2 holds when $E$ is the trivial local system in the case of an arbitrary field $\mathbb{k}$ of the same characteristic.

Proof. Let $s_{1}$ and $s_{2}$ be two $\overline{\mathbb{k}}$-points of $\operatorname{Bun}_{n}^{\prime}$, which project to the same point of $\mathrm{Bun}_{n}$. First, we can assume that all our data are defined over an algebra $A$ finitely generated over a finite field; $A \subset \overline{\mathbb{k}}$. In other words, we have the stacks $\left(\operatorname{Bun}_{n}\right)_{A}$ and $\left(\operatorname{Bun}_{n}^{\prime}\right)_{A}$ and sections $s_{i}: \operatorname{Spec} A \rightarrow\left(\operatorname{Bun}_{n}^{\prime}\right)_{A}$.

Consider $s_{i}^{*}\left(\right.$ Aut $\left._{E_{0}}^{\prime}\right), i=1,2$, where $E_{0}$ is the trivial local system (so that it is defined over $A$ ), as $\ell$-adic complexes on $\operatorname{Spec} A$. By localizing $A$ we may assume that it is smooth over a finite field, and that the above complexes are locally constant.

Let $\eta: \operatorname{Spec} \overline{\mathbb{k}} \rightarrow \operatorname{Spec} A$ be the canonical generic geometric point of Spec $A$, and let $a: \operatorname{Spec} \mathbb{F}_{q} \rightarrow \operatorname{Spec} A$ be some closed geometric point of Spec $A$. We need to compare the Euler characteristics of the stacks $\left(s_{i}^{*}\left(\text { Aut }_{E_{0}}^{\prime}\right)\right)_{\eta}$ for $i=1,2$. Since our complexes are locally constant, we may instead compare the stalks $\left(s_{i}^{*}\left(\operatorname{Aut}_{E}^{\prime}\right)\right)_{a}$. In other words, we can make the comparison over the finite field, as required 5

Thus, it suffices to prove Proposition 6.2 for the trivial local system in the case when $\mathbb{k}$ is a finite field.

Let us apply Lemma 6.6 again and obtain that it suffices to find just one local system $E^{s}$ in the case when $\mathbb{k}$ is a finite field, for which Proposition 6.2 is true. We will take as $E^{s}$ any irreducible local system, which satisfies the following conditions:

(a) $E^{s}$ is pure, and

(b) there exists a cuspidal Hecke eigenfunction associated to the pull-back of $E^{s}$ to $X \underset{\mathbb{F}_{q}}{\times \mathbb{F}_{q_{1}}}$ for any finite extension $\mathbb{F}_{q_{1}}$ of $\mathbb{F}_{q}$.

For example, such a local system can be constructed as follows: pick a cyclic $n$-sheeted étale cover $\widetilde{X} \rightarrow X$, and let $E^{s}$ be the direct image of a generic rank one local system on $\widetilde{X}$ of finite order. Then $E^{s}$ is pure, so condition (a) is satisfied. Moreover, according to Theorem 6.2 of $[\mathrm{AC}$ (see also $\mathrm{K}$ ), this local system also satisfies condition (b) 6

Thus, we have at our disposal at least one irreducible rank $n$ local system $E^{s}$, for which the above conditions (a), (b), as well as Conjecture 2.3 are true. We now prove that then Proposition 6.2 also holds for this $E^{s}$. To prove the first assertion of Proposition 6.2. it suffices to show that the function $\mathrm{f}_{q_{1}}\left(\mathrm{Aut}_{E^{s}}^{\prime}\right.$ ) (obtained by taking the traces of Frobenius on the stalks of $\mathrm{Aut}_{E^{s}}^{\prime}$; see Sect. 0.3$)$ on $\operatorname{Bun}_{n}^{\prime}\left(\mathbb{F}_{q_{1}}\right)$ is constant along the fibers of the projection

$$
\operatorname{Bun}_{n}^{\prime}\left(\mathbb{F}_{q_{1}}\right) \rightarrow \operatorname{Bun}_{n}\left(\mathbb{F}_{q_{1}}\right)
$$

for all finite extensions $\mathbb{F}_{q_{1}}$ of $\mathbb{F}_{q}$.

But Theorem 3.1 of [FGKV] states that if a cuspidal Hecke eigenfunction associated to any given rank $n$ local system $\widetilde{E}$ exists on $\operatorname{Bun}_{n}\left(\mathbb{F}_{q_{1}}\right)$, then its pull-back to $\operatorname{Bun}_{n}^{\prime}\left(\mathbb{F}_{q_{1}}\right)$ equals $\mathrm{f}_{q_{1}}\left(\right.$ Aut $\left._{\tilde{E}}^{\prime}\right)$ up to a non-zero scalar.

\footnotetext{
${ }^{5}$ Note that if the ground field $\mathbb{k}$ is of characteristic 0 , we can use a similar argument by choosing $A$ to be a finitely generated algebra over $\mathbb{Z}$.

${ }^{6}$ Actually, Lafforgue's results [Laf] imply that any irreducible local system $E$ satisfies conditions (a) and (b), up to a twist with a rank one local system.
} 
Applying these results to our local system $E^{s}$, we obtain that the function $\mathrm{f}_{q_{1}}\left(\mathrm{Aut}_{E^{s}}^{\prime}\right)$ is constant along the fibers of $\varrho_{n}: \operatorname{Bun}_{n}^{\prime}\left(\mathbb{F}_{q_{1}}\right) \rightarrow \operatorname{Bun}_{n}\left(\mathbb{F}_{q_{1}}\right)$. This proves the first assertion of Proposition 6.2 for $E^{s}$.

It remains to prove the non-vanishing assertion of Proposition 6.2 for $E^{s}$. According to Proposition 10.1, if $E$ is an irreducible local system on a curve $X$ over a finite field, which satisfies the above conditions (a) and (b), then Conjecture 2.3 holds for $E$. Hence by our assumptions on $E^{s}$, Conjecture 2.3 holds for $E^{s}$. Therefore by Theorem 3.7 the restriction of Aut $_{E^{s}}^{\prime}$ to the preimage of $\mathcal{C}_{n} \cap \operatorname{Bun}_{n}^{d}$ in $\operatorname{Bun}_{n}^{\prime}$ is a perverse sheaf, up to a cohomological shift. Hence it suffices to show that this restriction is non-zero (for if a perverse sheaf has zero Euler characteristics everywhere, then this sheaf is zero).

For that, it is enough to show that the corresponding function does not vanish identically on $\left(\mathrm{C}_{n} \cap \operatorname{Bun}_{n}^{d}\right)\left(\mathbb{F}_{q}\right)$, if $d \geq c_{g, n}$ (note that in this case $\mathcal{C}_{n} \cap \operatorname{Bun}_{n}^{d} \neq \emptyset$ ). However, by assumption,

$$
\operatorname{Bun}_{n}^{d}-\left(\mathcal{C}_{n} \cap \operatorname{Bun}_{n}^{d}\right) \subset \operatorname{Bun}_{n}^{\text {vuns }}, \quad d \geq c_{g, n}
$$

(see Sect. 3.2 for the definition of $\operatorname{Bun}_{n}^{\text {vuns }}$ ). The definition of cuspidal function implies the following

6.11. Lemma. Let $f$ be a cuspidal function on $\operatorname{Bun}_{n}\left(\mathbb{F}_{q}\right)$. Then its restriction to $\operatorname{Bun}_{n}^{\text {vuns }}\left(\mathbb{F}_{q}\right)$ is identically zero.

Proof. Let $\mathcal{M} \in \operatorname{Bun}_{n}\left(\mathbb{F}_{q}\right)$ be a very unstable bundle, and let $\mathcal{M} \simeq \mathcal{M}_{1} \oplus \mathcal{M}_{2}$ be the corresponding decomposition, with $\operatorname{rk}\left(\mathcal{M}_{i}\right)=n_{i}$.

Let $r_{n_{2}, n_{1}}^{n}: \operatorname{Funct}\left(\operatorname{Bun}_{n}\left(\mathbb{F}_{q}\right)\right) \rightarrow \operatorname{Funct}\left(\operatorname{Bun}_{n_{2}}\left(\mathbb{F}_{q}\right) \times \operatorname{Bun}_{n_{1}}\left(\mathbb{F}_{q}\right)\right)$ be the corresponding constant term operator. Since $f$ is cuspidal, we have $r_{n_{2}, n_{1}}^{n}(f)=0$. However, by applying the definition of $r_{n_{2}, n_{1}}^{n}$ and evaluating $r_{n_{2}, n_{1}}^{n}(f)$ at the point $\mathcal{M}_{2} \times \mathcal{M}_{1} \in \operatorname{Bun}_{n_{2}}\left(\mathbb{F}_{q}\right) \times \operatorname{Bun}_{n_{1}}\left(\mathbb{F}_{q}\right)$, we obtain that it is equal to the integral

$$
\int_{0 \rightarrow \mathcal{M}_{2} \rightarrow \mathcal{M}^{\prime} \rightarrow \mathcal{M}_{1} \rightarrow 0} f\left(M^{\prime}\right),
$$

over the finite set $\operatorname{Ext}^{1}\left(\mathcal{M}_{1}, \mathcal{M}_{2}\right)\left(\mathbb{F}_{q}\right)$ (the measure on this set is a non-zero multiple of the tautological measure).

However, by our assumption, $\operatorname{Ext}^{1}\left(\mathcal{M}_{1}, \mathcal{M}_{2}\right)=0$, therefore $r_{n_{2}, n_{1}}^{n}(f)\left(\mathcal{M}_{2}, \mathcal{M}_{1}\right)=$ $f(M)$, up to a non-zero constant.

Thus, we obtain the second assertion of Proposition 6.2 for our local system $E^{s}$. This completes the proof of Theorem 3.9.

\section{The Hecke Property of Aut A}

In the previous section we constructed a perverse sheaf $\mathcal{S}_{E}^{0}$ on $\mathcal{C}_{n}$, whose pull-back to $\mathcal{E}_{n}^{0}$ is $\mathcal{F}_{E, n} \otimes \overline{\mathbb{Q}}_{\ell}\left(\frac{n^{2} \cdot(g-1)}{2}\right)\left[n^{2} \cdot(g-1)\right]$.

Let $\mathcal{S}_{E}$ be the Goresky-MacPherson extension of $\mathcal{S}_{E}^{0}$ to $\bigcup_{d \geq c_{g, n}} \operatorname{Coh}_{n}^{d}$. Finally, set Aut $E:=\left.\mathcal{S}_{E}\right|_{\bigcup_{d \geq c, n}} \operatorname{Bun}_{n}^{d}$.

Our goal is to prove the following

7.1. Theorem. The perverse sheaf $\mathrm{Aut}_{E}$ can be uniquely extended to the entire stack $\mathrm{Bun}_{n}$, so that it becomes a Hecke eigensheaf with respect to $E$. 
Theorem [7.1] will follow from Proposition [7.7, as will be explained in Sect. 7.8 We will give two independent proofs of Proposition 7.7. The first one, presented in Sects. 7.2 7.6 uses the Whittaker sheaf $\mathcal{W}_{E}$. The second proof, given in Sect. 8 uses the Hecke-Laumon property of the Laumon sheaf $\mathcal{L}_{E}$.

7.2. The Hecke property on the stack $\operatorname{Bun}_{n}^{\prime}$. Consider the Cartesian product $\operatorname{Bun}_{n}^{\prime} \underset{\operatorname{Bun}_{n}}{\times} \mathcal{H}_{n}^{1}$, where the map $\mathcal{H}_{n}^{1} \rightarrow \operatorname{Bun}_{n}$ is $h \rightarrow$. We have a commutative diagram, in which the right square is Cartesian:

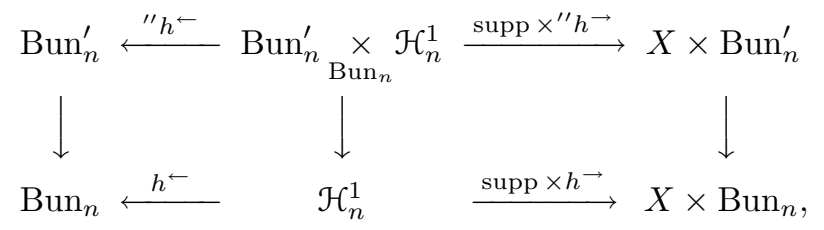

where the morphisms " $h \leftarrow$ and " $h \rightarrow$ are given by

${ }^{\prime \prime} h^{\leftarrow}:\left(x, \mathcal{M}, \mathcal{M}^{\prime}, \beta: \mathcal{M}^{\prime} \hookrightarrow \mathcal{M}, s^{\prime}: \Omega^{n-1} \rightarrow \mathcal{M}^{\prime}\right) \quad \mapsto\left(\mathcal{M}, s=\beta \circ s^{\prime}: \Omega^{n-1} \rightarrow \mathcal{M}\right)$,

${ }^{\prime \prime} h^{\rightarrow}: \quad\left(x, \mathcal{M}, \mathcal{M}^{\prime}, \beta: \mathcal{M}^{\prime} \hookrightarrow \mathcal{M}, s^{\prime}: \Omega^{n-1} \rightarrow \mathcal{M}^{\prime}\right) \quad \mapsto\left(\mathcal{M}^{\prime}, s^{\prime}\right)$.

7.3. Proposition. For any local system $E$ of rank $n$,

$$
\left(\operatorname{supp} \times^{\prime \prime} h^{\rightarrow}\right) !{ }^{\prime \prime} h^{\leftarrow *}\left(\mathrm{Aut}_{E}^{\prime}\right) \otimes \overline{\mathbb{Q}}_{\ell}\left(\frac{n-2}{2}\right)[n-2] \simeq E \otimes \mathrm{Aut}_{E}^{\prime} .
$$

First, we will reformulate this proposition in terms of the stack $\overline{\mathrm{Q}}^{d}$, introduced in Sect. 4.1.

7.4. A reformulation. We need to introduce two more stacks $\bar{Q}_{+}^{d}$ and $\bar{Q}_{++}^{d}$ closely related to $\overline{\mathrm{Q}}^{d}$. The stack $\overline{\mathrm{Q}}_{++}^{d}$ classifies the data of $\left(x, \mathcal{M},\left(s_{i}\right)\right)$ as in the definition of $\overline{\mathrm{Q}}^{d}$, but with

$$
s_{i}: \Omega^{(n-1)+\ldots+(n-i)} \rightarrow\left(\Lambda^{i} \mathcal{M}\right)(x), \quad i=1, \ldots, n .
$$

The stack $\bar{Q}_{+}^{d}$ classifies the same data with the additional condition that the image of $s_{1}$ is contained in $\mathcal{M}$ (and not just $\mathcal{M}(x)$ ). We have tautological closed embeddings

$$
X \times \overline{\mathrm{Q}}^{d} \hookrightarrow \overline{\mathrm{Q}}_{+}^{d} \hookrightarrow \overline{\mathrm{Q}}_{++}^{d} .
$$

Recall that we have a forgetful morphism $\overline{\mathrm{Q}}^{d+1} \rightarrow \mathrm{Bun}_{n}^{\prime}$, and the morphism $" h^{\leftarrow}: \operatorname{Bun}_{n}^{\prime} \underset{\operatorname{Bun}_{n}}{\times} \mathcal{H}_{n}^{1} \rightarrow \operatorname{Bun}_{n}^{\prime}$. Denote by $\bar{Q} \mathcal{H}^{d+1}$ the corresponding fiber product. Consider the following commutative diagram:

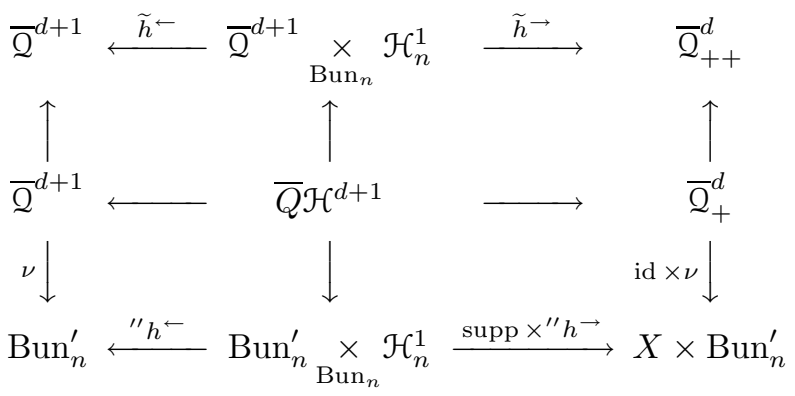

The bottom left and the top right squares in this diagram are Cartesian. 
By definition, Aut ${ }_{E}^{\prime}=\nu_{!}\left(\mathcal{W}_{E}\right)$, where $\nu: \overline{\mathrm{Q}} \rightarrow \operatorname{Bun}_{n}^{\prime}$ is the forgetful morphism defined in Sect. 4.3. Note that $\nu: \overline{\mathrm{Q}}^{d} \rightarrow \mathrm{Bun}_{n}^{\prime}$ extends to a morphism $\overline{\mathrm{Q}}_{+}^{d} \rightarrow$ $\operatorname{Bun}_{n}^{\prime}$, which we also denote by $\nu$. Using the diagram (7.3), we obtain that the LHS of formula (7.2), restricted to the degree $d$ connected component of $\mathrm{Bun}_{n}^{\prime}$, is isomorphic to the complex $(\mathrm{id} \times \nu) !\left(\mathcal{W}_{E,+}^{d}\right)$, where

$$
\mathcal{W}_{E,+}^{d}:=\left.\widetilde{h}_{!}^{\rightarrow} \widetilde{h}^{\leftarrow *}\left(\mathcal{W}_{E}^{d+1}\right)\right|_{\bar{\Omega}_{+}^{d}} \otimes \overline{\mathbb{Q}}_{\ell}\left(\frac{n-2}{2}\right)[n-2] .
$$

Therefore Proposition 7.3 follows from Proposition 7.5 which is proved in the Appendix.

7.5. Proposition. The complex $\mathcal{W}_{E,+}^{d}$ is supported on $X \times \overline{\mathrm{Q}}^{d} \subset \overline{\mathrm{Q}}_{+}^{d}$, and its restriction to $X \times \overline{\mathcal{Q}}^{d}$ is isomorphic to $E \otimes \mathcal{W}_{E}^{d}$.

7.6. The Hecke property on $\operatorname{Bun}_{n}$. Observe that in the diagram (1.1) defining the Hecke functor $\mathrm{H}_{n}^{1}$ we have:

$$
\left(\operatorname{supp} \times h^{\rightarrow}\right)^{-1}\left(X \times\left(\mathcal{C}_{n} \cap \operatorname{Bun}_{n}\right)\right) \subset\left(h^{\leftarrow}\right)^{-1}\left(\mathfrak{C}_{n} \cap \operatorname{Bun}_{n}\right) .
$$

Therefore we can define a functor $\mathrm{D}\left(\mathcal{C}_{n} \cap \mathrm{Bun}_{n}\right) \rightarrow \mathrm{D}\left(X \times\left(\mathcal{C}_{n} \cap \mathrm{Bun}_{n}\right)\right)$ by formula (1.2). We denote this functor also by $\mathrm{H}_{n}^{1}$ and consider its iterations $\left(\mathrm{H}_{n}^{1}\right)^{\otimes i}$ and the corresponding functors $\mathrm{H}_{n}^{i}$. The notion of Hecke eigensheaf also makes sense in this context.

We now derive from Proposition 7.3 the following

7.7. Proposition. The perverse sheaf $\mathrm{Aut}_{E} \mid \mathrm{e}_{n} \cap \mathrm{Bun}_{n}$ is a Hecke eigensheaf with respect to $E$.

Proof. Recall from Sect. 3.2 and Lemma 4.4 that over $\mathcal{E}_{n}^{0} \cap\left(\varrho_{n}\right)^{-1}\left(\operatorname{Bun}_{n}^{d}\right)$ we have an isomorphism

$$
\mathcal{F}_{E, n} \simeq \operatorname{Aut}_{E}^{\prime} \otimes \overline{\mathbb{Q}}_{\ell}\left(\frac{-d+c}{2}\right)[-d+c]
$$

where $c$ is a constant depending only on $g$ and $n$.

The isomorphism of $\mathrm{H}_{n}^{1}\left(\mathrm{Aut}_{E}\right)$ and $E \otimes \mathrm{Aut}_{E}$ over $\mathcal{C}_{n} \cap \mathrm{Bun}_{n}$ now follows from Proposition 7.3 via diagram (7.1), using the fact that the morphism $\rho_{n}^{0}$ : $\left(\operatorname{Bun}_{n}^{\prime} \cap \mathcal{E}_{n}^{0}\right) \rightarrow\left(\operatorname{Bun}_{n} \cap \mathcal{C}_{n}\right)$ is smooth, representable and has connected fibers.

Moreover, it follows from the construction of the isomorphism of Proposition 7.5 that this isomorphism satisfies condition (1.4).

Now we derive Theorem 7.1 from the above proposition.

7.8. Proof of Theorem 7.1. Recall the morphism mult $: X \times \operatorname{Bun}_{n} \rightarrow \operatorname{Bun}_{n}$ given by $(x, \mathcal{M}) \mapsto \mathcal{M}(x)$. In the same way as in the proof of Proposition 1.5, we obtain from Proposition 7.7 that there is an isomorphism

$$
\left.\left.\operatorname{mult}^{*}\left(\mathrm{Aut}_{E}\right)\right|_{X \times\left(\mathrm{e}_{n} \cap \mathrm{Bun}_{n}\right)} \simeq \Lambda^{n} E \otimes \operatorname{Aut}_{E}\right|_{X \times\left(\mathrm{e}_{n} \cap \mathrm{Bun}_{n}\right)} .
$$

Since the morphism mult : $X \times \operatorname{Bun}_{n} \rightarrow \operatorname{Bun}_{n}$ is smooth, the isomorphism of formula (7.5) holds over the entire component $\operatorname{Bun}_{n}^{d}$ for $d \geq c_{g, n}$ (and not only over $\left.\mathcal{C}_{n} \cap \operatorname{Bun}_{n}^{d}\right)$.

Now we extend $\mathrm{Aut}_{E}$ to all other connected components of $\mathrm{Bun}_{n}$ as follows: for every open substack $U \subset \operatorname{Bun}_{n}^{d^{\prime}}$ of finite type, there exists an integer $d^{\prime \prime}$ such that 
for any $x \in X$, the morphism mult ${ }_{d^{\prime \prime} \cdot x}: \operatorname{Bun}_{n} \rightarrow \operatorname{Bun}_{n}$ sending $\mathcal{M}$ to $\mathcal{M}\left(d^{\prime \prime} \cdot x\right)$ maps $U$ into $\mathcal{C}_{n} \cap \mathrm{Bun}_{n}$. We set $\left.\mathrm{Aut}_{E}\right|_{U}$ to be

$$
\operatorname{mult}_{d^{\prime \prime} \cdot x}^{*}\left(\operatorname{Aut}_{E}\right) \otimes\left(\Lambda^{n} E_{x}\right)^{\otimes-d^{\prime \prime}} .
$$

According to formula (7.5), this gives a well-defined sheaf Aut ${ }_{E}$ on the entire $\mathrm{Bun}_{n}$, together with an isomorphism mult ${ }^{*}\left(\mathrm{Aut}_{E}\right) \simeq \Lambda^{n} E \otimes \mathrm{Aut}_{E}$.

Proposition 7.7 then implies that $\mathrm{Aut}_{E}$ is a Hecke eigensheaf. Indeed, the existence and uniqueness of the isomorphism (1.3) satisfying (1.4) over the entire $\mathrm{Bun}_{n}$ follow from the construction, using the fact that formula (1.6) holds over $\mathcal{C}_{n} \cap \mathrm{Bun}_{n}$.

This completes the proof of Theorem 7.1

7.9. Lifting of $\mathrm{Aut}_{E}$ to $\mathrm{Bun}_{n}^{\prime}$. We have the sheaves $\mathrm{Aut}_{E}$ on $\mathrm{Bun}_{n}$ and $\mathrm{Aut}_{E}^{\prime}$ on $\operatorname{Bun}_{n}^{\prime}$. Consider the commutative diagram:

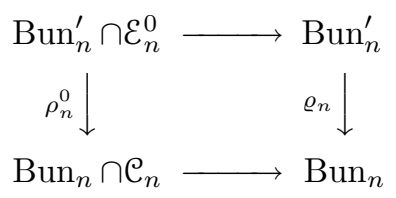

By construction, for $d \geq c_{n, g}$, the sheaves $\varrho_{n}{ }^{*}\left(\mathrm{Aut}_{E}\right)$ and $\mathrm{Aut}_{E}^{\prime} \otimes\left(\frac{-d+c}{2}\right)[-d+c]$ are isomorphic over $\mathcal{E}_{n}^{0} \cap\left(\varrho_{n}\right)^{-1}\left(\operatorname{Bun}_{n}^{d}\right)$, where $c$ is a constant independent of $d$. In this subsection we will address the following question, posed by V. Drinfeld:

Are the sheaves $\varrho_{n}{ }^{*}\left(\mathrm{Aut}_{E}\right)$ and $\mathrm{Aut}_{E}^{\prime} \otimes\left(\frac{-d+c}{2}\right)[-d+c]$ isomorphic on the entire $\operatorname{Bun}_{n}^{\prime}$ ?

The answer is affirmative. Indeed, consider the diagram

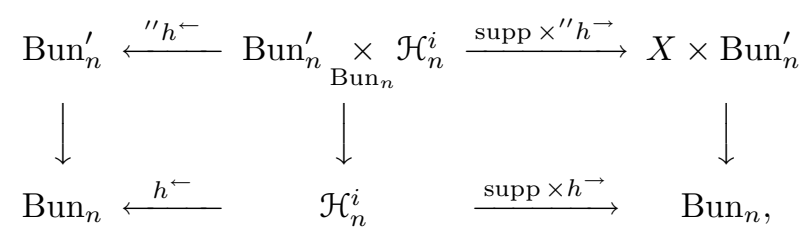

defined in the same way as diagram (7.1). From Proposition 7.3 we derive, in the same way as in Proposition 1.5, that

$$
\left(\operatorname{supp} \times^{\prime \prime} h^{\rightarrow}\right) ! !^{\prime \prime} h^{\leftarrow *}\left(\mathrm{Aut}_{E}^{\prime}\right) \otimes \overline{\mathbb{Q}}_{\ell}\left(\frac{i(n-i-1)}{2}\right)[i(n-i-1)] \simeq \Lambda^{i} E \otimes \operatorname{Aut}_{E}^{\prime} .
$$

In addition, from the Hecke property of $\mathrm{Aut}_{E}$ it follows that

$$
\left(\operatorname{supp} \times^{\prime \prime} h^{\rightarrow}\right) !^{\prime \prime} h^{\leftarrow *}\left(\varrho_{n}^{*}\left(\operatorname{Aut}_{E}\right)\right) \otimes \overline{\mathbb{Q}}_{\ell}\left(\frac{i(n-i)}{2}\right)[i(n-i)] \simeq \Lambda^{i} E \otimes \rho_{n}^{0 *}\left(\operatorname{Aut}_{E}\right) .
$$

As before, for $i=n$, the functor $\mathcal{K} \mapsto\left(\operatorname{supp} \times{ }^{\prime \prime} h^{\rightarrow}\right) !^{\prime \prime} h^{\leftarrow *}\left(\rho_{n}^{0 *}(\mathcal{K})\right)$ amounts to the pull-back under the map

$$
\operatorname{mult}^{\prime}: X \times \operatorname{Bun}_{n}^{\prime} \rightarrow \operatorname{Bun}_{n}^{\prime}
$$

given by

$$
\left(x, \mathcal{M}, s: \Omega^{n-1} \rightarrow \mathcal{M}\right) \mapsto\left(\mathcal{M}(x), s^{\prime}: \Omega^{n-1} \rightarrow \mathcal{M} \rightarrow \mathcal{M}(x)\right) .
$$

Any open substack $U$ of finite type in $\left(\varrho_{n}\right)^{-1}\left(\operatorname{Bun}_{n}^{d^{\prime}}\right)$ can be mapped into $\mathcal{E}_{n}^{0} \cap$ $\varrho_{n}^{-1}\left(\operatorname{Bun}_{n}^{d}\right)$ with $d \geq c_{g, n}$ by means of $\operatorname{mult}_{d^{\prime \prime} \cdot x}^{\prime}: \operatorname{Bun}_{n}^{\prime} \rightarrow \operatorname{Bun}_{n}^{\prime}$. Hence, over 
$\left(\varrho_{n}\right)^{-1}(U)$ we have:

$$
\begin{aligned}
& \operatorname{Aut}_{E}^{\prime} \otimes\left(\Lambda^{n} E_{x}\right)^{\otimes d^{\prime}} \otimes \overline{\mathbb{Q}}_{\ell}\left(\frac{-d^{\prime}+c}{2}\right)\left[-d^{\prime}+c\right] \\
& \simeq \operatorname{mult}_{d^{\prime \prime} \cdot x}^{\prime} *\left(\operatorname{Aut}_{E}^{\prime}\right) \otimes \overline{\mathbb{Q}}_{\ell}\left(\frac{-d^{\prime}-n \cdot d^{\prime \prime}+c}{2}\right)\left[-d^{\prime}-n \cdot d^{\prime \prime}+c\right] \\
& \simeq \operatorname{mult}_{d^{\prime \prime} \cdot x}^{\prime} *\left(\rho_{n}^{0 *}\left(\operatorname{Aut}_{E}\right)\right) \simeq \varrho_{n}^{*}\left(\operatorname{Aut}_{E}\right) \otimes\left(\Lambda^{n} E_{x}\right)^{\otimes d^{\prime \prime}} .
\end{aligned}
$$

The fact that the constructed isomorphism does not depend on the choice of $x$ and $d^{\prime}$ follows in the same way as the corresponding assertion for Aut ${ }_{E}$ in the proof of Theorem 7.1

\section{The Hecke-Laumon property of $\mathcal{S}_{E}$}

In this section we give an alternative proof of Proposition 7.7 and hence of Theorem 7.1 .

Consider the diagram

$$
\operatorname{Coh}_{n} \stackrel{h_{l}^{\leftarrow}}{\longleftarrow} \mathcal{H} \mathcal{L}_{n}^{d} \stackrel{h_{l}}{\longrightarrow} \operatorname{Coh}_{0}^{d} \times \operatorname{Coh}_{n},
$$

where the stack $\mathcal{H} \mathcal{L}_{n}^{d}$ classifies short exact sequences $0 \rightarrow \mathcal{M}^{\prime} \rightarrow \mathcal{M} \rightarrow \mathcal{T} \rightarrow 0$ with $\mathcal{M}^{\prime} \in \operatorname{Coh}_{n}, \mathcal{T} \in \operatorname{Coh}_{0}^{d}$. The projections $h_{l}^{\leftarrow}$ and $h_{l} \rightarrow$ send such data to $\mathcal{M}$ and $\left(\mathcal{M}^{\prime}, \mathcal{T}\right)$, respectively. (Recall that in Sect. 5.4 we encountered this stack for $n=0$ and called it $\mathrm{Fl}_{d}^{d^{\prime}, d^{\prime \prime}}$.)

The Hecke-Laumon functor $\mathrm{HL}_{n}^{d}: \mathrm{D}\left(\mathcal{C o}_{n}\right) \rightarrow \mathrm{D}\left(\operatorname{Coh}_{0}^{d} \times \operatorname{Coh}_{n}\right)$ is defined by the formula

$$
\left\{\begin{array}{l}
\mathrm{HL}_{n}^{d}(\mathcal{K})=h_{l} ! h_{l}^{\leftarrow *}(\mathcal{K}) \otimes \overline{\mathbb{Q}}_{\ell}\left(\frac{d \cdot(n+1)}{2}\right)[d \cdot(n+1)], n \geq 1 \\
\mathrm{HL}_{0}^{d}(\mathcal{K})=h_{l} ! h_{l}^{\leftarrow *}(\mathcal{K})
\end{array}\right.
$$

(see [Lau2]).

Note that for $d=d_{1}+d_{2}$ there is a natural isomorphism of functors

$$
\left(\mathrm{id} \times \mathrm{HL}_{n}^{d_{2}}\right) \circ \mathrm{HL}_{n}^{d_{1}} \simeq\left(\mathrm{HL}_{0}^{d_{1}} \times \mathrm{id}\right) \circ \mathrm{HL}_{n}^{d}
$$

Finally, let us note that (5.8) stated in Sect. 5.4 reads as

$$
\mathrm{HL}_{0}^{d_{1}}\left(\mathcal{L}_{E}^{d}\right) \simeq \mathcal{L}_{E}^{d_{1}} \otimes \mathcal{L}_{E}^{d_{2}} .
$$

8.1. Definition. We say that a complex $\mathcal{K} \in \mathrm{D}\left(\operatorname{Coh}_{n}\right)$ has a Hecke-Laumon property (or is a Hecke-Laumon eigensheaf) with respect to $E$ if for each $d$ we are given an isomorphism

$$
\mathrm{HL}_{n}^{d}(\mathcal{K}) \simeq \mathcal{L}_{E}^{d} \otimes \mathcal{K}
$$

such that for $d=d_{1}+d_{2}$ the diagram

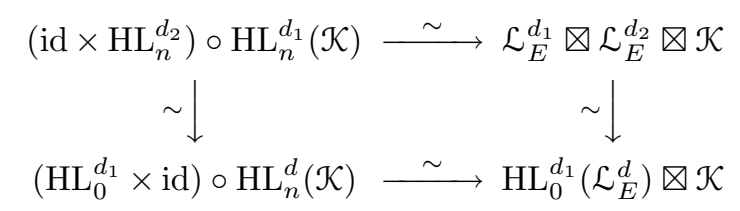

is commutative. 
8.2. Restriction to $\mathcal{C}_{n}$. Note that the definition of the Hecke-Laumon property makes sense not only on $\mathcal{C o h}_{n}$, but also on $\mathcal{C}_{n}$. Consider the stack $\left(\mathcal{H} \mathcal{L}_{n}^{d}\right)^{\prime}:=$ $\mathcal{H} \mathcal{L}_{n}^{d} \underset{\operatorname{Coh}_{0}^{d} \times \mathcal{C o h}_{n}}{\times} \operatorname{Coh}_{0}^{d} \times \mathcal{E}_{n}^{0}$ and note that it fits into the diagram:

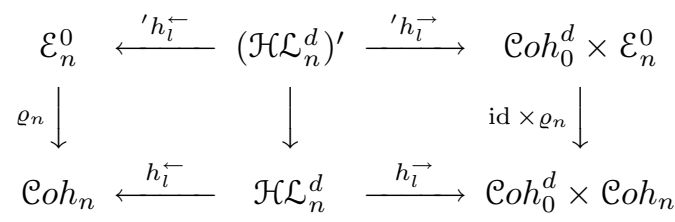

It is shown in Lau2 (by induction on $k$ ) that

$$
{ }^{\prime} h_{l} !_{!}^{\prime} h_{l}^{\leftarrow}\left(\mathcal{F}_{E, n}\right) \otimes \overline{\mathbb{Q}}_{\ell}\left(\frac{d \cdot(n+1)}{2}\right)[d \cdot(n+1)] \simeq \mathcal{L}_{E}^{d} \otimes \mathcal{F}_{E, n}
$$

Therefore, since $\rho_{n}^{0}: \mathcal{E}_{n}^{0} \rightarrow \mathcal{C}_{n}$ is smooth, representable and with connected fibers, we obtain:

8.3. Corollary. The perverse sheaf $\mathcal{S}_{E}^{0}$ on $\mathcal{C}_{n}$ is a Hecke-Laumon eigensheaf with respect to $E$.

We will now prove the following result:

8.4. Proposition. Let $\mathcal{S}$ be a perverse sheaf on $\operatorname{Coh}_{n}$, and let $\mathbb{D}(\mathcal{S})$ be its Verdier dual sheaf. Suppose that $\mathcal{S}$ and $\mathbb{D}(\mathcal{S})$ satisfy the Hecke-Laumon property with respect to local systems $E$ and $E^{*}$, respectively. Then $\mathcal{K}:=\left.\mathcal{S}\right|_{\operatorname{Bun}_{n}}$ is a Hecke eigensheaf with respect to $E$.

Proof. We start with the following general observation. Let $\rho: \mathcal{E} \rightarrow Y$ be a vector bundle, let $i: Y \rightarrow \mathcal{E}$ be the zero section, and let $j: \mathcal{E}^{0} \rightarrow \mathcal{E}$ be its complement. Let us denote by $\widetilde{\rho}: \mathbb{P} \mathcal{E} \rightarrow Y$ the corresponding projectivized bundle.

Suppose that $\mathcal{F}$ is a $\mathbb{G}_{m}$-equivariant perverse sheaf on $\mathcal{E}$, and set $\mathcal{F}^{0}:=\left.\mathscr{F}\right|_{\mathcal{E}^{0}}$. We will denote by $\widetilde{\mathcal{F}}$ the perverse sheaf on $\mathbb{P} \mathcal{E}$ corresponding to $\mathcal{F}^{0}$, i.e., the pull-back of $\widetilde{\mathcal{F}}$ to $\mathcal{E}^{0}$ is $\mathcal{F}^{0} \otimes \overline{\mathbb{Q}}_{\ell}\left(\frac{-1}{2}\right)[-1]$. We have the following assertion (see [Ga]).

8.5. Lemma. Assume that $\rho_{*}(\mathcal{F})[-1]$ and $\rho_{!}(\mathcal{F})[1]$ are perverse sheaves. Then $\widetilde{\rho}_{!}(\widetilde{\mathcal{F}})$ is a perverse sheaf as well, and $\rho_{!}(\mathcal{F}) \otimes \overline{\mathbb{Q}}_{\ell}\left(\frac{1}{2}\right)[1] \simeq \widetilde{\rho}_{!}(\widetilde{\mathcal{F}}) \simeq \rho_{*}(\mathcal{F}) \otimes \overline{\mathbb{Q}}_{\ell}\left(\frac{-1}{2}\right)[-1]$.

Proof. Since $\mathcal{F}$ is $\mathbb{G}_{m}$-equivariant, $\rho_{!}(\mathcal{F}) \simeq i^{!}(\mathcal{F})$ and $\rho_{*}(\mathcal{F}) \simeq i^{*}(\mathcal{F})$. By applying $i^{!}$ to the triangle

$$
j_{!} \mathcal{F}^{0} \rightarrow \mathcal{F} \rightarrow i_{*} i^{*}(\mathcal{F})
$$

we obtain that $\rho_{!} j_{!}\left(\mathcal{F}^{0}\right) \simeq i^{!} j_{!}\left(\mathcal{F}^{0}\right)$ has perverse cohomology only in cohomological degrees 0 and 1.

Using the Leray spectral sequence of the composition $\mathcal{E}^{0} \rightarrow \mathbb{P} \mathcal{E} \rightarrow Y$, we obtain that $\widetilde{\rho}_{!}(\widetilde{\mathcal{F}})$ must be perverse. In addition, we obtain that $\widetilde{\rho}_{!}(\widetilde{\mathcal{F}}) \simeq h^{0}\left(\rho_{!} j_{!}\left(\mathcal{F}^{0}\right)\right) \otimes$ $\overline{\mathbb{Q}}_{\ell}\left(\frac{-1}{2}\right)$, which identifies $\widetilde{\rho}_{!}(\widetilde{\mathcal{F}})$ with $i^{*}(\mathcal{F}) \otimes \overline{\mathbb{Q}}_{\ell}\left(\frac{-1}{2}\right)[-1]$. Similarly, we obtain: $\widetilde{\rho}_{!}(\widetilde{\mathcal{F}}) \simeq i^{!}(\mathcal{F}) \otimes \overline{\mathbb{Q}}_{\ell}\left(\frac{1}{2}\right)[1]$.

We will reduce the assertion of Proposition 8.4 to the above lemma. Set $Y=$ $\operatorname{Coh}_{0}^{1} \times \operatorname{Bun}_{n} \subset \operatorname{Coh}_{0}^{1} \times \operatorname{Coh}_{n}$ and take $\mathcal{E}$ to be

$$
\left(h_{l}\right)^{-1}\left(\operatorname{Coh}_{0}^{1} \times \operatorname{Bun}_{n}\right) \subset \mathcal{H} \mathcal{L}_{n}^{1} .
$$


(Note that in general the preimage $\left(h_{l} \rightarrow\right)^{-1}\left(\operatorname{Coh}_{0}^{d} \times \operatorname{Bun}_{n}\right) \subset \mathcal{H} \mathcal{L}_{n}^{d}$ is the same as the stack $\widetilde{\operatorname{Coh}}_{n, \leq d}$ introduced in Sect. 5.2 and the map $\widetilde{\operatorname{Coh}}_{n, \leq d} \hookrightarrow \mathcal{H} \mathcal{L}_{n}^{d} \stackrel{h_{l}^{\leftarrow}}{\longrightarrow} \operatorname{Coh}_{n}$ becomes the map $r: \widetilde{\operatorname{Coh}}_{n, \leq d} \rightarrow \operatorname{Coh}_{n, \leq d} \subset \operatorname{Coh}_{n}$.)

Set $\mathcal{F}=h_{l}^{\leftarrow *}(\mathcal{S}) \otimes \overline{\mathbb{Q}}_{\ell}\left(\frac{n}{2}\right)[n]$. Then $\mathcal{F}$ is $\mathbb{G}_{m}$-equivariant and perverse, according to Lemma 5.3. In addition, the image of $\mathcal{E}^{0}$ under $h_{l}^{\leftarrow}$ lies in $\operatorname{Bun}_{n} \subset \operatorname{Coh}_{n}$.

By the assumption of Proposition 8.4, $\mathcal{S}$ is a Hecke-Laumon eigensheaf. This implies that $\rho_{!}(\mathcal{F}) \otimes \overline{\mathbb{Q}}_{\ell}\left(\frac{1}{2}\right)[1] \simeq \mathcal{L}_{E}^{1} \otimes \mathcal{K}$, and so $\rho_{!}(\mathcal{F})[1]$ is a perverse sheaf. Applying Verdier duality and using the assumptions of Proposition 8.4 regarding $\mathbb{D}(\mathcal{S})$, we obtain that $\rho_{*}(\mathcal{F})[-1]$ is a perverse sheaf too. Hence, we can apply Lemma 8.5 .

Let us perform a base change with respect to $X \rightarrow \mathcal{C o h}_{0}^{1}$. Then $X \underset{\operatorname{Coh}_{0}^{1}}{\times} \mathbb{P} \mathcal{E}$ identifies naturally with the Hecke correspondence $\mathcal{H}_{n}^{1}$ in such a way that

$$
\widetilde{\rho}: X \underset{\operatorname{Coh}_{0}^{1}}{\times} \mathbb{P} \mathcal{E} \rightarrow X \times \operatorname{Bun}_{n}
$$

becomes the projection $h \rightarrow$. Therefore, Lemma 8.5 implies that $\mathrm{H}_{n}^{1}(\mathcal{K}) \simeq E \nabla \mathcal{K}$.

The fact that this isomorphism indeed satisfies condition (1.4) follows from property (8.4) in the case $d=2$. This completes the proof of Proposition 8.4.

8.6. Remark. V. Drinfeld has asked the following question about the possibility of proving a theorem converse to Proposition 8.4.

Let $\mathcal{K}$ be a perverse sheaf on $\mathrm{Bun}_{n}$, which is a Hecke eigensheaf with respect to $E$. Is it true that the Goresky-MacPherson extension of $\mathcal{K}$ to Coh $_{n}$ has the Hecke-Laumon property with respect to $E$ ?

We conjecture that the answer to this question is affirmative.

8.7. Second proof of Proposition 1.7. It is clear from the above proof that

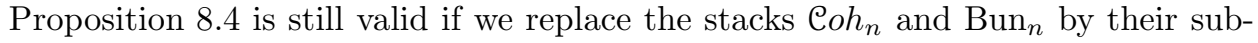
stacks $\mathcal{C}_{n}$ and $\mathcal{C}_{n} \cap \mathrm{Bun}_{n}$, respectively. Now we apply this modification of Proposition 8.4 in the situation when $\mathcal{S}=\mathcal{S}_{E}^{0}$, and $\mathcal{K}=$ Aut $_{E} \mid \mathcal{C}_{n} \cap \mathrm{Bun}_{n}$. It follows from the definitions that all conditions of Proposition 8.4 are satisfied (in particular, we have: $\left.\mathbb{D}\left(\mathcal{S}_{E}^{0}\right) \simeq \mathcal{S}_{E^{*}}^{0}\right)$. The statement of Proposition 7.7 (and hence Theorem 7.1 ) now follows directly from Proposition 8.4 .

8.8. Lifting of $\mathcal{S}_{E}$ to $\operatorname{Coh}_{n}^{\prime}$. We have the diagram:

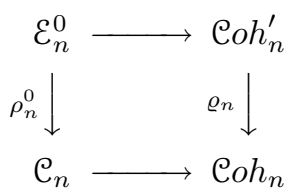

Recall the definition of the perverse sheaf $\mathcal{S}_{E}$ on $\bigcup_{d \geq c_{n, g}} \operatorname{Coh}_{n}^{d}$ given in the beginning of Sect. [7. By Theorem 3.9 and Lemma 3.5] over $\left(\varrho_{n}\right)^{-1}\left(\mathcal{C}_{n} \cap\left(\bigcup_{d \geq c_{n, g}} \operatorname{Coh}_{n}^{d}\right)\right)$ the complexes $\varrho_{n}^{*}\left(\mathcal{S}_{E}\right)$ and $\mathcal{S}_{E}^{\prime} \otimes \overline{\mathbb{Q}}_{\ell}\left(\frac{-d+c}{2}\right)[-d+c]$ are isomorphic, where $c$ is a constant independent of $d$.

Consider the Goresky-MacPherson extension of Aut $_{E}$ (which by now is defined on the whole of $\mathrm{Bun}_{n}$ ) to $\operatorname{Coh}_{n}$. By abuse of notation we still denote this extension by $\mathcal{S}_{E}$. Now we prove the following assertion:

The sheaf $\mathcal{S}_{E}$ has the Hecke-Laumon property with respect to $E$, and $\varrho_{n}{ }^{*}\left(\mathcal{S}_{E}\right) \simeq$ $\mathcal{S}_{E}^{\prime} \otimes \overline{\mathbb{Q}}_{\ell}\left(\frac{-d+c}{2}\right)[-d+c]$. 
Let us denote by $\left(X \times \operatorname{Coh}_{n}\right)^{0}$ the open substack of $X \times \operatorname{Coh}_{n}$ corresponding to those pairs $(x, \mathcal{N})$ for which $\mathcal{M}$ has no torsion supported at $x$. In a similar way we define the substack $\left(X^{i} \times \operatorname{Coh}_{n}\right)^{0}$ of $X^{i} \times \operatorname{Coh}_{n}$. We define the functors

$$
\mathrm{H}_{n}^{i}: \mathrm{D}\left(\operatorname{Coh}_{n}\right) \rightarrow \mathrm{D}\left(\left(X \times \operatorname{Coh}_{n}\right)^{0}\right)
$$

in the same way as before. Since we already know Aut $_{E}$ is a Hecke eigensheaf, we obtain that

$$
\left.\mathrm{H}_{n}^{n}\left(\mathcal{S}_{E}\right) \simeq \Lambda^{n}(E) \otimes \mathcal{S}_{E}\right|_{\left.\left(X^{i} \times \mathcal{C}_{o}\right)_{n}\right)^{0}}
$$

By arguing as in Sect. 7.8, we deduce the Hecke-Laumon property of $\mathcal{S}_{E}$ on the entire $\mathrm{Coh}_{n}$ from (8.6) and the fact that $\mathcal{S}_{E} \mid \mathrm{e}_{n}$ is a Hecke-Laumon sheaf.

Similarly, the isomorphism $\varrho_{n}{ }^{*}\left(\mathcal{S}_{E}\right) \simeq \mathcal{S}_{E}^{\prime} \otimes \overline{\mathbb{Q}}_{\ell}\left(\frac{-d+c}{2}\right)[-d+c]$ follows in the same way as in Sect. 7.9.

\section{Cuspidality}

9.1. Constant term functors. Let $P \subset G L_{n}$ be the standard (upper) parabolic subgroup corresponding to a partition $\left(n_{1}, \ldots, n_{k}\right)$ of $n$, with the Levi quotient $M \simeq G L_{n_{1}} \times \ldots \times G L_{n_{k}}$. The embedding of $P$ in $G L_{n}$ and the projection $P \rightarrow M$ induce morphisms $p$ and $q$ in the diagram

$$
\operatorname{Bun}_{n} \stackrel{\mathfrak{p}}{\longleftarrow} \operatorname{Bun}_{P} \stackrel{\mathfrak{q}}{\longrightarrow} \operatorname{Bun}_{M}
$$

The constant term functor $\mathrm{R}_{M}^{G}: \mathrm{D}\left(\mathrm{Bun}_{n}\right) \rightarrow \mathrm{D}\left(\mathrm{Bun}_{M}\right)$ is defined by the formula $\mathrm{R}_{M}^{G}(\mathcal{K})=q ! p^{*}(\mathcal{K})$. We say that $\mathcal{K} \in \mathrm{D}\left(\operatorname{Bun}_{n}\right)$ is cuspidal if $\mathrm{R}_{M}^{G}(\mathcal{F})=0$ for all proper parabolic subgroups $P$ of $G$.

For a partition $n=n_{1}+n_{2}$ let $P\left(n_{1}, n_{2}\right)$ be the corresponding parabolic subgroup in $G L_{n}$ with the Levi factor $G L_{n_{1}} \times G L_{n_{2}}$. In this case diagram (9.1) is

$$
\operatorname{Bun}_{n} \stackrel{\mathfrak{p}}{\leftarrow} \operatorname{Bun}_{P\left(n_{1}, n_{2}\right)} \stackrel{\mathfrak{q}}{\longrightarrow} \operatorname{Bun}_{n_{1}} \times \operatorname{Bun}_{n_{2}} .
$$

We denote the corresponding constant term functor $\mathrm{D}\left(\mathrm{Bun}_{n}\right) \rightarrow \mathrm{D}\left(\mathrm{Bun}_{n_{1}} \times \mathrm{Bun}_{n_{2}}\right)$ by $\mathrm{R}_{n_{1}, n_{2}}^{n}(\mathcal{K})$.

It is easy to see that a complex $\mathcal{K}$ is cuspidal if and only if $\mathrm{R}_{n_{1}, n_{2}}^{n}(\mathcal{K})=0$ for all partitions $n=n_{1}+n_{2}$, with $n_{1}, n_{2}>0$.

In this section we prove the following

9.2. Theorem. Let $\mathrm{Aut}_{E}$ be a Hecke eigensheaf on $\mathrm{Bun}_{n}$ with respect to an irreducible rank $n$ local system $E$, which satisfies Conjecture 2.3. Then Aut $_{E}$ is cuspidal.

As a corollary we obtain the following statement:

9.3. Corollary. The perverse sheaf $\mathrm{Aut}_{E}$ is the extension by zero from an open substack of finite type on every connected component of $\mathrm{Bun}_{n}$.

Proof. The proof of the corollary will rely on the following well-known assertion:

9.4. Lemma. For a fixed line bundle $\mathcal{L}$ and an integer $d$, consider the open substack $U$ of $\operatorname{Bun}_{n}^{d}$ which classifies vector bundles $\mathcal{M}$ with $\operatorname{Hom}(\mathcal{M}, \mathcal{L})=0$. Then $U$ is of finite type.

To prove the corollary, let us consider a connected component $\operatorname{Bun}_{n}^{d}$. Without loss of generality we may assume that $d \geq c_{g, n}$. Take $\mathcal{L}=\mathcal{L}^{\text {est }}$ and let $U$ be as in the lemma. By definition, $\operatorname{Bun}_{n}^{d}-U$ is contained in $\operatorname{Bun}_{n}^{\text {vuns }}$. 
However, by arguing as in Lemma 6.11 we obtain that if a complex $\mathcal{K}$ is cuspidal, then it has zero stalks at all very unstable bundles.

Therefore, $\left.\mathcal{K}\right|_{\operatorname{Bun}_{n}^{d}}$ is extended by zero from $U$. This completes the proof of Corollary 9.3.

The proof of Theorem 9.2 as well as other results of this section relies on the following computation:

9.5. Proposition. Let $\mathcal{K}$ be a Hecke eigensheaf with respect to some rank $n$ local system $E^{\prime}$, and let $E$ be another local system, of an arbitrary rank. Then

$$
\mathrm{H}_{n, E}^{d}(\mathcal{K}) \simeq \mathcal{K} \otimes H^{\bullet}\left(X^{(d)},\left(E \otimes E^{\prime *}\right)^{(d)}\right) \otimes \overline{\mathbb{Q}}_{\ell}\left(\frac{d}{2}\right)[d] .
$$

Proof. Consider the diagram

$$
\operatorname{Bun}_{n} \stackrel{h^{\leftarrow}}{\longleftarrow} \operatorname{Mod}_{n}^{d} \stackrel{\operatorname{supp} \times h^{\rightarrow}}{\longrightarrow} X^{(d)} \times \operatorname{Bun}_{n} .
$$

We need to prove that

$$
\left(\operatorname{supp} \times h^{\rightarrow}\right) !\left(h^{\leftarrow *}(\mathcal{K}) \otimes \pi^{*}\left(\mathcal{L}_{E}^{d}\right)\right) \otimes\left(\overline{\mathbb{Q}}_{\ell}\left(\frac{1}{2}\right)[1]\right)^{\otimes d \cdot(n-1)} \simeq\left(E \otimes E^{* *}\right)^{(d)} \otimes \mathcal{K} .
$$

Consider the stack $\widetilde{\operatorname{Mod}}_{n}^{d}$, which classifies the data $\left(\mathcal{M}=\mathcal{M}_{0} \subset \mathcal{M}_{1} \subset \ldots \subset\right.$ $\mathcal{M}_{i}=\mathcal{M}^{\prime}$ ), where each $\mathcal{M}_{j}$ is a rank $n$ vector bundle, and $\mathcal{M}_{j} / \mathcal{M}_{j-1}$ is a simple skyscraper sheaf. (Note that there is a canonical isomorphism between $\widetilde{\operatorname{Mod}_{n}^{d}}$ and the stack $\widehat{\operatorname{Mod}}_{n}^{-d}$, introduced in the proof of Theorem 1.5, under which the roles of the projections $h^{\leftarrow}$ and $h^{\rightarrow}$ get reversed.)

Let $p: \widehat{\operatorname{Mod}}_{n}^{d} \rightarrow \operatorname{Mod}_{n}^{d}$ be the forgetful map. We also have a natural morphism $\widetilde{\operatorname{supp}}: \widetilde{\operatorname{Mod}}_{n}^{d} \rightarrow X^{d}$. Consider the corresponding diagram:

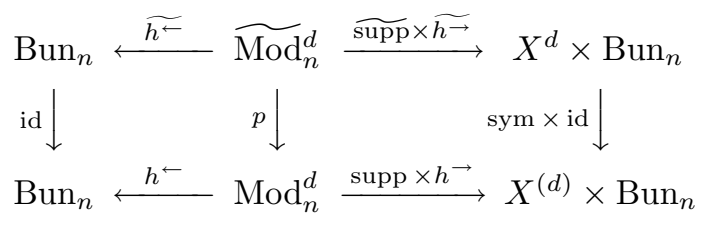

Since $p$ is small, the complex $p_{\text {! }} \widetilde{\operatorname{supp}}^{*}\left(E^{\bigotimes d}\right)$ is a perverse sheaf (up to a cohomological shift), which is the Goresky-MacPherson extension of its own restriction to $\operatorname{supp}^{-1}\left(X^{(d)}-\Delta\right)$. In particular, it carries a canonical action of the symmetric group $S_{d}$ and

$$
\left(p_{!} \widetilde{\operatorname{supp}}^{*}\left(E^{\bigotimes d}\right)\right)^{S_{d}} \simeq \pi^{*}\left(\mathcal{L}_{E}^{d}\right) .
$$

As was noted before, the stack $\operatorname{Mod}_{n}^{1}$ is isomorphic to the stack $\mathcal{H}_{n}^{1}$, but under this isomorphism the maps $h^{\leftarrow}$ and $h^{\rightarrow}$ become interchanged. By iterating the definition of the Hecke property, we obtain that the fact that $\mathcal{K}$ is a Hecke eigensheaf with respect to $E^{\prime}$ then implies that

$$
\left(\widetilde{\operatorname{supp}} \times \widetilde{h^{\rightarrow}}\right) ! \widetilde{h^{\leftarrow}} *(\mathcal{K}) \otimes \overline{\mathbb{Q}}_{\ell}\left(\frac{n-1}{2}\right)[n-1] \simeq\left(E^{\prime *}\right)^{\otimes d} \otimes \mathcal{K} .
$$

Hence we obtain:

$$
\left(\widetilde{\operatorname{supp}} \times \widetilde{h^{\rightarrow}}\right) !\left(\widetilde{h^{\leftarrow}} *(\mathcal{K}) \otimes \widetilde{\operatorname{supp}}^{*}\left(E^{\otimes d}\right)\right) \otimes\left(\overline{\mathbb{Q}}_{\ell}\left(\frac{1}{2}\right)[1]\right)^{\otimes d \cdot(n-1)} \simeq\left(E \otimes E^{\prime *}\right)^{\otimes d} \otimes \mathcal{K} .
$$


By taking the direct image of the last isomorphism under sym : $X^{d} \rightarrow X^{(d)}$ we obtain

$$
\begin{aligned}
(\operatorname{supp} & \left.\times h^{\rightarrow}\right)_{!}\left(h^{\leftarrow *}(\mathcal{K}) \otimes p_{!} \widetilde{\operatorname{supp}}^{*}\left(E^{\bigotimes d}\right)\right) \otimes\left(\overline{\mathbb{Q}}_{\ell}\left(\frac{1}{2}\right)[1]\right)^{\otimes d \cdot(n-1)} \\
& \simeq \operatorname{sym}_{!}\left(\left(E \otimes E^{\prime *}\right)^{\bigotimes d}\right) \otimes \mathcal{K} .
\end{aligned}
$$

Moreover, this isomorphism is compatible with the $S_{d}$-action on both sides.

By passing to the $S_{d}$-invariants we obtain formula (9.2) and hence the statement of the proposition.

9.6. Remark. Let us see what the isomorphism of formula (9.2) looks like at the level of fibers over a given point $D \in X^{(d)}$, in terms of the general Hecke functors $\mathrm{H}_{n}^{\lambda}$ introduced in Sect. A.1. To simplify notation we take $D=d \cdot x$, for some $x \in X$.

By formula (A.4) below, the stalk of the LHS of formula (9.2) at $D$ can be identified with

$$
\bigoplus_{\lambda \in P_{n, d}^{+}}{ }_{x} \mathrm{H}_{n}^{-w_{0}(\lambda)}(\mathcal{K}) \otimes E_{x}^{\lambda}
$$

Since $\mathcal{K}$ is a Hecke eigensheaf with respect to $E^{\prime}$, this is isomorphic to

$$
\bigoplus_{\lambda \in P_{n, d}^{+}} \mathcal{K} \otimes\left(E^{* *}\right)_{x}^{\lambda} \otimes E_{x}^{\lambda} \simeq \mathcal{K} \otimes \operatorname{Sym}^{d}\left(E_{x} \otimes E_{x}^{*}\right)
$$

which is the stalk of the RHS of formula (9.2) at $d \times x$.

9.7. Remark. Recall the stack $\operatorname{Mod}_{k}^{-d}$ introduced in the proof of Theorem 1.5 Denote by $\mathrm{H}_{k, E}^{-d}$ the functor

$$
\mathcal{K} \mapsto h_{!} \rightarrow\left(h^{\leftarrow *}(\mathcal{K}) \otimes \pi^{*}\left(\mathcal{L}_{E}^{d}\right)\right) \otimes \overline{\mathbb{Q}}_{\ell}\left(\frac{d \cdot n}{2}\right)[d \cdot n]
$$

(where $h^{\leftarrow}$ and $h \rightarrow$ are taken according to the definition of $\operatorname{Mod}_{k}^{-d}$ ). It follows from the definition that the functor $\mathrm{H}_{k, E}^{-d}$ is both left and right adjoint to $\mathrm{H}_{k, E^{*}}^{d}$.

In the same way as in the proof of Proposition 9.5 we obtain:

$$
\mathrm{H}_{n, E}^{-d}(\mathcal{K}) \simeq \mathcal{K} \otimes H^{\bullet}\left(X^{(d)},\left(E \otimes E^{\prime}\right)^{(d)}\right) \otimes \overline{\mathbb{Q}}_{\ell}\left(\frac{d}{2}\right)[d]
$$

Now we are ready to prove Theorem 9.2 .

9.8. Lemma. For each $d, n=n_{1}+n_{2}$, a local system $E$ and $\mathcal{K} \in \mathrm{D}\left(\mathrm{Bun}_{n}\right)$, the object $\mathrm{R}_{n_{1}, n_{2}}^{n} \circ \mathrm{H}_{n, E}^{d}(\mathcal{K}) \in \mathrm{D}\left(\operatorname{Bun}_{n_{1}} \times \operatorname{Bun}_{n_{2}}\right)$ has a canonical filtration by the objects $\left(\mathrm{H}_{n_{1}, E}^{d_{1}} \times \mathrm{H}_{n_{2}, E}^{d_{2}}\right) \circ \mathrm{R}_{n_{1}, n_{2}}^{n}(\mathcal{K}) \otimes \overline{\mathbb{Q}}_{\ell}\left(\frac{-n_{1} \cdot d_{2}}{2}\right)\left[-n_{1} \cdot d_{2}\right]$ for all possible partitions $d=d_{1}+d_{2}$ with $d_{1}, d_{2} \geq 0$.

9.9. Proof of Theorem 9.2. Theorem 9.2 follows from Lemma 9.8 Indeed, take $d>2 n^{2}(2 g-2)$. On the one hand, according to Proposition 9.5.

$$
\mathrm{H}_{n, E}^{d}\left(\mathrm{Aut}_{E}\right) \simeq \operatorname{Aut}_{E} \otimes H^{\bullet}\left(X^{(d)},\left(E \otimes E^{*}\right)^{(d)}\right),
$$

hence

$$
\mathrm{R}_{n_{1}, n_{2}}^{n} \circ \mathrm{H}_{n, E}^{d}\left(\mathrm{Aut}_{E}\right) \simeq \mathrm{R}_{n_{1}, n_{2}}^{n}\left(\operatorname{Aut}_{E}\right) \otimes H^{\bullet}\left(X^{(d)},\left(E \otimes E^{*}\right)^{(d)}\right) \otimes \overline{\mathbb{Q}}_{\ell}\left(\frac{d}{2}\right)[d] .
$$

On the other hand, Conjecture 2.3 implies that all $\left(\mathrm{H}_{n_{1}, E}^{d_{1}} \times \mathrm{H}_{n_{2}, E}^{d_{2}}\right) \circ \mathrm{R}_{n_{1}, n_{2}}^{n}\left(\mathrm{Aut}_{E}\right)$ must vanish, because either $d_{1}$ or $d_{2}$ must be greater than $n^{2}(2 g-2)$. 
However, since $E \otimes E^{*}$ contains the trivial rank one local system,

$$
H^{\bullet}\left(X^{(d)},\left(E \otimes E^{*}\right)^{(d)}\right) \neq 0 \text { for any } d .
$$

Hence, $\mathrm{R}_{n_{1}, n_{2}}^{n}\left(\operatorname{Aut}_{E}\right)=0$. This completes the proof of Theorem 9.2 ,

9.10. Proof of Lemma 9.8 Let $\operatorname{Mod}_{n}^{d} \underset{\operatorname{Bun}_{n}}{\times} \operatorname{Bun}_{P\left(n_{1}, n_{2}\right)}$ be the Cartesian product defined using the projection $h \rightarrow: \operatorname{Mod}_{n}^{d} \rightarrow \operatorname{Bun}_{n}$. Our task is to calculate the direct image under

$$
\operatorname{Mod}_{n}^{d} \underset{\operatorname{Bun}_{n}}{\times} \operatorname{Bun}_{P\left(n_{1}, n_{2}\right)} \stackrel{h^{\rightarrow} \times \text { id }}{\longrightarrow} \operatorname{Bun}_{P\left(n_{1}, n_{2}\right)} \stackrel{\mathfrak{q}}{\rightarrow} \operatorname{Bun}_{n_{1}} \times \operatorname{Bun}_{n_{2}}
$$

of the pull-back under $\operatorname{Mod}_{n}^{d} \underset{\operatorname{Bun}_{n}}{\times} \operatorname{Bun}_{P\left(n_{1}, n_{2}\right)} \stackrel{\text { id } \times \mathfrak{p}}{\longrightarrow} \operatorname{Mod}_{n}$ of the complex $h^{\leftarrow *}(\mathcal{K}) \otimes$ $\pi^{*}\left(\mathcal{L}_{E}^{d}\right)$.

By definition, the above Cartesian product classifies the data of

$$
\begin{aligned}
& \mathcal{M} \in \operatorname{Bun}_{n}, \mathcal{M}^{\prime} \in \operatorname{Bun}_{n}, \beta: \mathcal{M}^{\prime} \hookrightarrow \mathcal{M}, \\
& \mathcal{M}_{1} \in \operatorname{Bun}_{n_{1}}, \mathcal{M}_{2} \in \operatorname{Bun}_{n_{2}}, 0 \rightarrow \mathcal{M}_{1} \rightarrow \mathcal{M} \rightarrow \mathcal{M}_{2} \rightarrow 0 .
\end{aligned}
$$

First, we decompose $\operatorname{Mod}_{n}^{d} \underset{\operatorname{Bun}_{n}}{\times} \operatorname{Bun}_{P\left(n_{1}, n_{2}\right)}$ into locally closed substacks, which we will denote by

$$
\left(\operatorname{Mod}_{n}^{d} \underset{\operatorname{Bun}_{n}}{\times} \operatorname{Bun}_{P\left(n_{1}, n_{2}\right)}\right)^{d_{1}, d_{2}} .
$$

By definition, a point of $\operatorname{Mod}_{n}^{d} \underset{\operatorname{Bun}_{n}}{\times} \operatorname{Bun}_{P\left(n_{1}, n_{2}\right)}$ as in formula (9.4) belongs to $\left(\operatorname{Mod}_{n}^{d} \underset{\operatorname{Bun}_{n}}{\times} \operatorname{Bun}_{P\left(n_{1}, n_{2}\right)}\right)^{d_{1}, d_{2}}$ if we have $\operatorname{deg}\left(\mathcal{M}^{\prime} \cap \mathcal{M}_{1}\right)=\operatorname{deg}\left(\mathcal{M}_{1}\right)-d_{1}$. From each $\left(\operatorname{Mod}_{n}^{d} \underset{\operatorname{Bun}_{n}}{\times} \operatorname{Bun}_{P\left(n_{1}, n_{2}\right)}\right)^{d_{1}, d_{2}}$ there is a natural map to $\operatorname{Mod}_{n_{1}}^{d_{1}} \times \operatorname{Mod}_{n_{2}}^{d_{2}}$, which sends a point as above to $\left(\mathcal{M}_{1}^{\prime}:=\mathcal{M}^{\prime} \cap \mathcal{M}_{1} \hookrightarrow \mathcal{M}_{1}, \mathcal{M}_{2}^{\prime}:=\mathcal{M}^{\prime} / \mathcal{M}_{1}^{\prime} \hookrightarrow \mathcal{M}_{2}\right)$.

To prove the proposition it suffices to show that the direct image under this map of the complex that we obtain on $\left(\operatorname{Mod}_{n}^{d} \underset{\operatorname{Bun}_{n}}{\times} \operatorname{Bun}_{P\left(n_{1}, n_{2}\right)}\right)^{d_{1}, d_{2}}$ by restriction from $\operatorname{Mod}_{n}^{d} \underset{\operatorname{Bun}_{n}}{\times} \operatorname{Bun}_{P\left(n_{1}, n_{2}\right)}$ can be canonically identified with $\left(h^{\leftarrow} \times h^{\leftarrow}\right)^{*}\left(\mathrm{R}_{n_{1}, n_{2}}^{n}(\mathcal{K})\right) \otimes$ $(\pi \times \pi)^{*}\left(\mathcal{L}_{E}^{d_{1}} \otimes \mathcal{L}_{E}^{d_{2}}\right) \otimes \overline{\mathbb{Q}}_{\ell}\left(\frac{-n_{1} \cdot d_{2}}{2}\right)\left[-n_{1} \cdot d_{2}\right]$ in the diagram

$$
\operatorname{Bun}_{n_{1}} \times \operatorname{Bun}_{n_{2}} \stackrel{h^{\leftarrow} \times h^{\leftarrow}}{\longleftarrow} \operatorname{Mod}_{n_{1}}^{d_{1}} \times \operatorname{Mod}_{n_{2}}^{d_{2}} \stackrel{\pi \times \pi}{\longrightarrow} \operatorname{Coh}_{0}^{d_{1}} \times \operatorname{Coh}_{0}^{d_{2}} .
$$

For that purpose, we decompose the map

$$
\left(\operatorname{Mod}_{n}^{d} \underset{\operatorname{Bun}_{n}}{\times} \operatorname{Bun}_{P\left(n_{1}, n_{2}\right)}\right)^{d_{1}, d_{2}} \rightarrow \operatorname{Mod}_{n_{1}}^{d_{1}} \times \operatorname{Mod}_{n_{2}}^{d_{2}}
$$

as a composition of several ones. First, we introduce the stack $y_{1}$, which classifies the data of

$$
\begin{aligned}
& 0 \rightarrow \mathcal{M}_{1}^{\prime} \rightarrow \mathcal{M}_{1} \rightarrow \mathcal{T}_{1} \rightarrow 0, \mathcal{M}_{1} \in \operatorname{Bun}_{n_{1}}, \mathcal{T}_{1} \in \operatorname{Coh}_{0}^{d_{1}}, \\
& 0 \rightarrow \mathcal{M}_{2}^{\prime} \rightarrow \mathcal{M}_{2} \rightarrow \mathcal{T}_{2} \rightarrow 0, \mathcal{M}_{2} \in \operatorname{Bun}_{n_{2}}, \mathcal{T}_{1} \in \operatorname{Coh}_{0}^{d_{2}}, \\
& 0 \rightarrow \mathcal{T}_{1} \rightarrow \mathcal{T} \rightarrow \mathcal{T}_{2} \rightarrow 0,0 \rightarrow \mathcal{M}_{1}^{\prime} \rightarrow \mathcal{M}^{\prime} \rightarrow \mathcal{M}_{2}^{\prime} \rightarrow 0 .
\end{aligned}
$$

It is easy to see that the natural map $\left(\operatorname{Mod}_{n}^{d} \underset{\operatorname{Bun}_{n}}{\times} \operatorname{Bun}_{P\left(n_{1}, n_{2}\right)}\right)^{d_{1}, d_{2}} \rightarrow y_{1}$ is a fibration into affine spaces, with each fiber being a principal homogeneous space for $\operatorname{Ext}^{1}\left(\mathcal{T}^{2}, \mathcal{M}_{1}^{\prime}\right)$. Therefore, by the projection formula, the direct image of our 
complex to $y_{1}$ is the pull-back under the map $y_{1} \rightarrow \operatorname{Bun}_{n} \times \operatorname{Coh}_{0}^{d}$ (which sends a point as above to $\left.\left(\mathcal{M}^{\prime}, \mathcal{T}\right)\right)$ of $\mathcal{K} \otimes \mathcal{L}_{E}^{d} \otimes \overline{\mathbb{Q}}_{\ell}\left(\frac{-n_{1} \cdot d_{2}}{2}\right)\left[-n_{1} \cdot d_{2}\right]$.

Now, let $y_{2}$ be the stack classifying the data of

$$
\begin{aligned}
& 0 \rightarrow \mathcal{M}_{1}^{\prime} \rightarrow \mathcal{M}_{1} \rightarrow \mathcal{T}_{1} \rightarrow 0, \mathcal{M}_{1} \in \operatorname{Bun}_{n_{1}}, \mathcal{T}_{1} \in \operatorname{Coh}_{0}^{d_{1}}, \\
& 0 \rightarrow \mathcal{M}_{2}^{\prime} \rightarrow \mathcal{M}_{2} \rightarrow \mathcal{T}_{2} \rightarrow 0, \mathcal{M}_{2} \in \operatorname{Bun}_{n_{2}}, \mathcal{T}_{2} \in \operatorname{Coh}_{0}^{d_{2}}, \\
& 0 \rightarrow \mathcal{M}_{1}^{\prime} \rightarrow \mathcal{M}^{\prime} \rightarrow \mathcal{M}_{2}^{\prime} \rightarrow 0 .
\end{aligned}
$$

The projection $y_{1} \rightarrow y_{2}$ corresponds to forgetting the class of the extension $0 \rightarrow$ $\mathcal{T}_{1} \rightarrow \mathcal{T} \rightarrow \mathcal{T}_{2} \rightarrow 0$. Moreover, we have a Cartesian square:

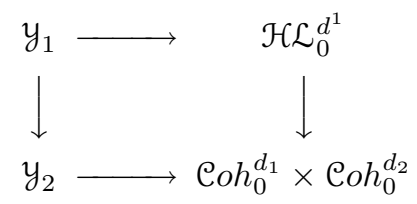

Using the projection formula and the fact that $\mathrm{HL}_{0}^{d_{1}}\left(\mathcal{L}_{E}^{d}\right) \simeq \mathcal{L}_{E}^{d_{1}} \otimes \mathcal{L}_{E}^{d_{2}}$, we obtain that direct image under $y_{1} \rightarrow y_{2}$ of the pull-back of $\mathcal{K} \otimes \mathcal{L}_{E}^{d}$ is the tensor product of the pull-back of $\mathcal{K}$ under the map $y_{2} \rightarrow \operatorname{Bun}_{n}$, which sends a point as above to $\mathcal{M}^{\prime}$ and the pull-back of $\mathcal{L}_{E}^{d_{1}} \otimes \mathcal{L}_{E}^{d_{2}}$ under the natural map from $y_{2}$ to $\operatorname{Coh}_{0}^{d_{1}} \times \operatorname{Coh}_{0}^{d_{2}}$.

Finally, note that we have a Cartesian square:

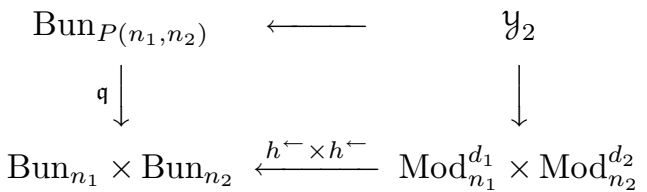

where the upper horizontal arrow sends a point of $y_{2}$ as above to $0 \rightarrow \mathcal{M}_{1}^{\prime} \rightarrow \mathcal{M}^{\prime} \rightarrow$ $\mathcal{M}_{2}^{\prime} \rightarrow 0$. The assertion follows now by the projection formula.

\section{Proof of the Vanishing Conjecture over $\mathbb{F}_{q}$}

In this section we prove Conjecture 2.3 in the case when the ground field $\mathbb{k}$ is a finite field $\mathbb{F}_{q}$, i.e., that the functor $\mathrm{H}_{k, E}^{d}: \mathrm{D}\left(\mathrm{Bun}_{k}\right) \rightarrow \mathrm{D}\left(\mathrm{Bun}_{k}\right)$ introduced in Sect. 2.2 is identically zero if $E$ is an irreducible local system of rank $n$, and $k$ and $d$ satisfy the inequalities $k<n$ and $d>k n(2 g-2)$.

Namely, we will prove the following proposition:

10.1. Proposition. Let $E$ be a rank $n$ local system on $X$ over the finite field $\mathbb{F}_{q}$, which is

(a) pure up to a twist by a one-dimensional representation of the Weil group of $\mathbb{F}_{q}$,

and satisfies one of the following conditions:

(b) there exists a cuspidal Hecke eigenfunction associated to the pull-back of $E$ to

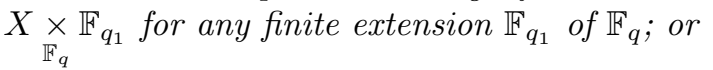

$\left(\mathrm{b}^{\prime}\right)$ the space of unramified cuspidal automorphic functions on the group $G L_{k}$ over the adèles is spanned by the Hecke eigenfunctions corresponding to rank

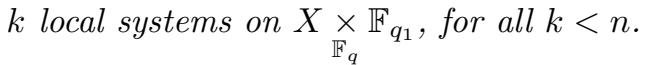

Then the Vanishing Conjecture 2.3 holds for E. 
According to [Laf], Theorem VII.6, any irreducible local system $E$, such that $\operatorname{det} E$ is of finite order, is pure. Therefore condition (a) of Proposition [10.1 is satisfied for any irreducible rank $n$ local system $E$. Moreover, both statements (b) and $\left(\mathrm{b}^{\prime}\right)$ hold for such $E$, according to the main theorem of Lafforgue's work. Hence we obtain that the Vanishing Conjecture 2.3 is true for all irreducible local systems if the ground field $\mathbb{k}$ is finite.

Our proof of Proposition 10.1 proceeds as follows. We first show vanishing of $\mathrm{H}_{k, E}^{d}$ at the level of functions. Using the purity property conjectured by Deligne and proved by Lafforgue [Laf], we will then deduce that $\mathrm{H}_{k, E}^{d}(\mathcal{K})=0$ for any $\mathcal{K} \in \mathrm{D}\left(\operatorname{Bun}_{k}\right), k=1, \ldots, n-1, d>k n(2 g-2)$.

10.2. $L$-functions. Let $\Gamma^{(1)}=\left(\gamma_{x}^{(1)}\right)_{x \in|X|}$ and $\Gamma^{(2)}=\left(\gamma_{x}^{(2)}\right)_{x \in|X|}$ be two collections of semi-simple conjugacy classes in $G L_{k}\left(\overline{\mathbb{Q}}_{\ell}\right)$ and $G L_{n}\left(\overline{\mathbb{Q}}_{\ell}\right)$, respectively. We attach to it the $L$-function

$$
L\left(\Gamma^{(1)}, \Gamma^{(2)}, t\right)=\prod_{x \in|X|} \operatorname{det}\left(\operatorname{Id}_{k n}-\left(\gamma_{x}^{(1)} \otimes \gamma_{x}^{(2)}\right) t^{\operatorname{deg} x}\right)^{-1}
$$

viewed as a formal power series in $t$.

To an unramified irreducible representation $\pi=\bigotimes_{x \in X}^{\prime} \pi_{x}$ of $G L_{k}(\mathbb{A})$, where $\mathbb{A}$ is the ring of adèles of $F=\mathbb{F}_{q}(X)$, we attach the collection $\Gamma_{\pi}=\left(s_{x}\right)_{x \in|X|}$, where $s_{x}$ is the Satake parameter of $\pi_{x}$

If $\pi$ and $\pi^{\prime}$ are unramified irreducible representations $\pi$ of $G L_{k}(\mathbb{A})$ and $G L_{n}(\mathbb{A})$, respectively, we write

$$
L\left(\pi \times \pi^{\prime}, t\right):=L\left(\Gamma_{\pi}, \Gamma_{\pi^{\prime}}, t\right) .
$$

If $\pi$ and $\pi^{\prime}$ are in addition cuspidal automorphic representations, then $L\left(\pi \times \pi^{\prime}, t\right)$ is the Rankin-Selberg $L$-function of the pair $\pi, \pi^{\prime}$. The following statement follows from results of [PS2, CPS] (see [Laf], Appendice B, for a review) 7

10.3. Theorem. If $\pi, \pi^{\prime}$ are cuspidal automorphic representations and $k<n$, then $L\left(\pi \times \pi^{\prime}, t\right)$ is a polynomial of degree $k n(2 g-2)$.

Next, we attach to a rank $n$ local system $E$ on $X$ the collection of conjugacy classes $\Gamma_{E}=\left(\left.\operatorname{Fr}_{x}\right|_{E_{x}}\right)_{x \in|X|}$.

If $E$ and $E^{\prime}$ are two local systems on $X$, of ranks $n$ and $k$, respectively, we write:

$$
L\left(E^{\prime} \times E, t\right):=L\left(\Gamma_{E^{\prime}}, \Gamma_{E}, t\right) .
$$

10.4. Lemma. If both $E$ and $E^{\prime}$ are irreducible and $k<n$, then $L\left(E^{\prime} \times E\right.$, $\left.t\right)$ is a polynomial of degree $k n(2 g-2)$.

Proof. Using the definition of $L\left(E^{\prime} \times E, t\right)$ and the Grothendieck-Lefschetz formula, we obtain:

$$
L\left(E^{\prime} \times E, t\right)=\sum_{d \geq 0} \operatorname{Tr}\left(\mathrm{Fr}, H^{d}\left(X^{(d)},\left(E^{\prime} \otimes E\right)^{(d)}\right)\right) t^{d} .
$$

Since $E^{\prime} \otimes E$ is irreducible by our assumptions, $H^{0}\left(E^{\prime} \otimes E\right)=H^{2}\left(E^{\prime} \otimes E\right)=0$. Therefore,

$$
H^{d}\left(X^{(d)},\left(E^{\prime} \otimes E\right)^{(d)}\right) \simeq \Lambda^{d}\left(H^{1}\left(X, E^{\prime} \otimes E\right)\right)=0
$$

for all $d>\operatorname{dim} H^{1}\left(X^{(d)}, E^{\prime} \otimes E\right)=k n(2 g-2)$.

${ }^{7}$ We are grateful to V. Drinfeld for pointing this out to us. 
If $E$ is a rank $n$ local system on $X$, and $\pi$ is an irreducible unramified representation of $G L_{k}(\mathbb{A})$, we will write

$$
L(\pi \times E, t):=L\left(\Gamma_{\pi}, \Gamma_{E}, t\right) .
$$

10.5. Computation of $\mathrm{H}_{k, E}^{d}$ at the level of functions. The functor $\mathrm{H}_{k, E}^{d}$ gives rise to a linear map on the space of functions on the set $\operatorname{Bun}_{k}\left(\mathbb{F}_{q}\right)$ of $\mathbb{F}_{q}$-points of $\operatorname{Bun}_{k}$. We denote this operator by $\mathbf{H}_{k, E}^{d}$. In this subsection we will prove that $\mathbf{H}_{k, E}^{d} \equiv 0$ for all $k=1, \ldots, n-1$ and $d>k n(2 g-2)$.

Recall that $\operatorname{Bun}_{k}\left(\mathbb{F}_{q}\right)$ is naturally identified with the double quotient

$$
G L_{k}(F) \backslash G L_{n}(\mathbb{A}) / G L_{n}(\mathcal{O})
$$

(see, e.g., FGKV], Sect. 2). Let $\pi$ be a cuspidal unramified automorphic representation of $G L_{k}(\mathbb{A})$. Attached to it is a cuspidal automorphic function on $\operatorname{Bun}_{k}\left(\mathbb{F}_{q}\right)$, unique up to a non-zero scalar multiple. We normalize it in some way and denote the result by $f_{\pi}$.

In Remark 9.7 we defined the functor $\mathrm{H}_{k, E}^{-d}$, which is left and right adjoint to $\mathrm{H}_{k, E^{*}}^{d}$. Denote by $\mathbf{H}_{k, E}^{-d}$ the corresponding linear map on the space of functions on $\operatorname{Bun}_{k}\left(\mathbb{F}_{q}\right)$. We have the following analogue of formula (9.3), which is proved using a calculation similar to the one presented in the proof of Proposition 9.5.

$$
\sum_{d \geq 0} \mathbf{H}_{k, E}^{-d}\left(f_{\pi}\right) \cdot t^{d}=L(\pi \times E, t) \cdot f_{\pi} .
$$

It is clear from the definition that

$$
\left\langle\mathbf{H}_{k, E}^{d}(f), f^{\prime}\right\rangle=\left\langle f, \mathbf{H}_{k, E}^{-d}\left(f^{\prime}\right)\right\rangle,
$$

where the inner product of two automorphic functions $f_{1}, f_{2}$ on $G L_{k}(\mathbb{A})$ is defined by the formula

$$
\left\langle f_{1}, f_{2}\right\rangle=\int_{G L_{k}(F) \backslash G L_{k}(\mathbb{A})} f_{1}(g) f_{2}(g) d g .
$$

Formula (10.1) then implies:

$$
\left\langle\mathbf{H}_{k, E}^{d}(f), f_{\pi}\right\rangle=L(\pi \times E, t)\left\langle f, f_{\pi}\right\rangle .
$$

10.6. Lemma. Let $E$ be a rank $n$ local system such that $L(\pi \times E, t)$ is a polynomial of degree $k n(2 g-2)$ for all irreducible cuspidal automorphic representations $\pi$ of $G L_{k}(\mathbb{A}), k=1, \ldots, n-1$. Then $\mathbf{H}_{k, E}^{d}(f)=0$ for any function $f$ on $\operatorname{Bun}_{k}\left(\mathbb{F}_{q}\right)$.

Proof. By induction, we may assume that the assertion is known for $k^{\prime}<k$. In the same way as in the proof Theorem 9.2 we then obtain that $\mathbf{H}_{k, E}^{d}(f)$ is a cuspidal function for any function $f$ on $\operatorname{Bun}_{k}\left(\mathbb{F}_{q}\right)$. By formula [10.3), if $L\left(\pi \times E^{*}, t\right)$ is a polynomial of degree $k n(2 g-2)$, then $\left\langle\mathbf{H}_{k, E}^{d}(f), f^{\prime}\right\rangle=0$ for any function $f$, any cuspidal automorphic function $f^{\prime}$ and all $d>k n(2 g-2)$. Therefore $\mathbf{H}_{k, E}^{d}(f)=$ 0 .

Thus, in order to prove vanishing of $\mathbf{H}_{k, E}^{d}$ for $k=1, \ldots, n-1$ and $d>k n(2 g-2)$, we need to show that $L(\pi \times E, t)$ is a polynomial of degree $k n(2 g-2)$ for all irreducible cuspidal automorphic representations $\pi$ of $G L_{k}(\mathbb{A})$ for $k=1, \ldots, n-1$. This can be done in two ways: by identifying $L(\pi \times E, t)$ with $L\left(\pi \times \pi^{\prime}, t\right)$ or with $L\left(E^{\prime} \times E, t\right)$. As the result, we obtain that $\mathbf{H}_{k, E}^{d} \equiv 0, k=1, \ldots, n-1$, if either of the statements (b) or $\left(\mathrm{b}^{\prime}\right)$ listed in Proposition 10.1 is true. 
Indeed, if the statement (b) is true, then there exists an unramified cuspidal automorphic representation $\pi^{\prime}$ of $G L_{n}(\mathbb{A})$, such that $L(\pi \times E, t)=L\left(\pi \times \pi^{\prime}, t\right)$. Vanishing of $\mathbf{H}_{k, E}^{d}$ for $k<n$ and $d>k n(2 g-2)$ then follows from Theorem 10.3

If the statement $\left(b^{\prime}\right)$ is true, then vanishing follows from Lemma 10.4

10.7. Conclusion of the proof of Proposition 10.1. To complete the proof of Proposition 10.1 we need to show that vanishing of the operator $\mathbf{H}_{k, E}^{d}$ at the level of functions implies the vanishing of the operator $\mathrm{H}_{k, E}^{d}$ at the level of sheaves, provided that $E$ is pure. In order to do that, we proceed as follows: for each $x:$ Spec $\mathbb{F}_{q} \rightarrow$ Bun $_{k}$, denote by $\delta_{x}$ the direct image with compact support of the constant sheaf on $\operatorname{Spec} \mathbb{F}_{q}$. Proving that the functor $\mathrm{H}_{k, E}^{d}$ vanishes is equivalent to showing that $\mathrm{H}_{k, E}^{d}\left(\delta_{x}\right)=0$, for all $x$.

Since $\operatorname{Bun}_{k}$ is a stack (and not a scheme), $\delta_{x}$ is not necessarily an irreducible perverse sheaf, but it is a mixed complex. Therefore, it suffices to show that $\mathrm{H}_{k, E}^{d}(\mathcal{K})=0$, for any mixed complex $\mathcal{K}$. Decomposing $\mathrm{H}_{k, E}^{d}(\mathcal{K})$ in the derived category, we obtain that it is enough to show that $\mathrm{H}_{k, E}^{d}(\mathcal{K})=0$, when $\mathcal{K}$ is a pure perverse sheaf.

Now let $E$ be a pure irreducible rank $n$ local system on $X$. Then Laumon's sheaf $\mathcal{L}_{E}$ is also pure. The pull-back with respect to a smooth morphism preserves purity, and so does the push-forward with respect to a proper representable morphism (see [BBD] $)$. But the morphism $\left(h^{\leftarrow} \times \pi\right): \operatorname{Mod}_{n}^{d} \rightarrow \operatorname{Bun}_{n} \times \operatorname{Coh}_{0}^{d}$ is smooth, and the $\operatorname{morphism} h \rightarrow: \operatorname{Mod}_{n}^{d} \rightarrow \operatorname{Bun}_{n}$ is proper and representable. Hence $\mathrm{H}_{k, E}^{d}(\mathcal{K})$ is pure, if $\mathcal{K}$ is pure.

The function $\mathrm{f}_{q_{1}}\left(\mathrm{H}_{k, E}^{d}(\mathcal{K})\right)$ associated to the sheaf $\mathrm{H}_{k, E}^{d}(\mathcal{K})$ equals $\mathbf{H}_{k, E}^{d}\left(\mathbf{f}_{q_{1}}(\mathcal{K})\right)$ for any for $q_{1}=q^{r}, r \in \mathbb{Z}_{>0}$ (here we use the notation introduced in Sect.0.3). But according to the computation of Sect. 10.5, $\mathbf{H}_{k, E}^{d}\left(\mathrm{f}_{q_{1}}(\mathcal{K})\right)=0$ for all $k=1, \ldots, n-1$ and $d>k n(2 g-2)$ if either of the conditions (b) or $\left(\mathrm{b}^{\prime}\right)$ of Proposition 10.1 holds for $E$. In addition, we have:

10.8. Lemma. A pure complex $\mathcal{F}$ vanishes if and only if the corresponding function $\mathbf{f}_{q_{1}}(\mathcal{F})$ is zero, for all $q_{1}=q^{r}, r \in \mathbb{Z}_{>0}$.

Proof. Since $\mathcal{F}$ is non-zero, there exists a locally closed subset $U$ such that $\left.\mathcal{F}\right|_{U}$ is locally constant and non-zero. Since $\mathcal{F}$ is pure, $\left.\mathcal{F}\right|_{U}$ is pointwise pure. But for a pointwise pure non-zero locally constant complex, all functions $\mathrm{f}_{q_{1}}\left(\left.\mathcal{F}\right|_{U}\right)$ cannot be identically equal to zero for all $q_{1}=q^{r}, r \in \mathbb{Z}_{>0}$, by the condition on the absolute values of the Frobenius eigenvalues.

Therefore the statement of Conjecture 2.3 holds for $E$. This completes the proof of Proposition 10.1.

10.9. Remark. Formula 10.2) has a geometric counterpart:

$$
\left\langle\mathrm{H}_{k, E}^{d}(\mathcal{K}), \mathcal{K}^{\prime}\right\rangle \simeq\left\langle\mathcal{K}, \mathrm{H}_{k, E^{*}}^{-d}\left(\mathcal{K}^{\prime}\right)\right\rangle, \quad \mathcal{K}, \mathcal{K}^{\prime} \in \mathrm{D}\left(\operatorname{Bun}_{k}\right),
$$

where $\left\langle\mathcal{K}, \mathcal{K}^{\prime}\right\rangle:=\operatorname{RHom}\left(\mathcal{K}, \mathcal{K}^{\prime}\right)$. Note that a priori $\operatorname{RHom}\left(\mathcal{K}, \mathcal{K}^{\prime}\right)$ makes sense if $\mathcal{K}$ is the !-extension from a substack of $\operatorname{Bun}_{k}$ of finite type, or $\mathcal{K}^{\prime}$ is the ${ }^{*}$-extension from a substack of $\operatorname{Bun}_{k}$, whose intersection with every connected component is of finite type.

Let $E$ and $E^{\prime}$ be two irreducible local systems on $X$, of ranks $n$ and $k$, respectively, where $k<n$. Let us assume that the Vanishing Conjecture 2.3 holds for $E^{\prime}$. 
In particular, Aut $E_{E^{\prime}}$ exists and is cuspidal, according to Theorem 9.2. Therefore, for every $d$, Aut $\left._{E^{\prime}}\right|_{\mathrm{Bun}_{k}^{d}}$ is extended by zero from an open substack of finite type of $\operatorname{Bun}_{n}^{d}$.

From formula (9.3) we obtain the following analogue of formula (10.3):

$$
\left\langle\mathrm{H}_{k, E}^{d}(\mathcal{K}), \operatorname{Aut}_{E^{\prime}}\right\rangle \simeq H^{d}\left(X^{(d)},\left(E^{*} \otimes E^{\prime}\right)^{(d)}\right) \otimes\left\langle\mathcal{K}, \operatorname{Aut}_{E^{\prime}}\right\rangle \otimes \overline{\mathbb{Q}}_{\ell}\left(\frac{d}{2}\right)[d] .
$$

Since $H^{d}\left(X^{(d)},\left(E^{*} \otimes E^{\prime}\right)^{(d)}\right)=0$ for $d>k n(2 g-2)$ (see the proof of Lemma 10.4), we find that

$$
\left\langle\mathrm{H}_{k, E}^{d}(\mathcal{K}), \operatorname{Aut}_{E^{\prime}}\right\rangle=0,
$$

for all $\mathcal{K} \in \mathrm{D}\left(\operatorname{Bun}_{k}\right)$, if $d>k n(2 g-2)$. Thus we obtain a geometric analogue of Proposition 10.1

10.10. Proposition. Suppose that for $k=1, \ldots, n-1$ the Vanishing Conjecture 2.3 is true for rank $k$ local systems on $X$ and in addition the following statement holds:

$\left(\mathrm{b}^{\prime \prime}\right)$ If $\mathcal{F} \in \mathrm{D}\left(\mathrm{Bun}_{k}\right)$ is cuspidal and satisfies $\left\langle\mathcal{F}, \mathrm{Aut}_{E^{\prime}}\right\rangle=0$ for all irreducible rank $k$ local system $E^{\prime}$ on $X$, then $\mathcal{F}=0$.

Then the Vanishing Conjecture 2.3 is true for any irreducible local system on $X$ of rank $n$.

The above statement $\left(\mathrm{b}^{\prime \prime}\right)$ is known to be true for $k=1$ in the case when char $\mathbb{k}=0$, by the Fourier-Mukai transform [Lau3, $\mathbb{R}$.

\section{Appendix A. Hecke functors and Whittaker sheaves}

A.1. General Hecke functors. We recall some results from Sect. 5 of [FGV].

Let ${ }_{x} \mathcal{H}_{n}$ be the full Hecke correspondence stack at $x \in|X|$. In other words, ${ }_{x} \mathcal{H}_{n}$ classifies triples $\left(\mathcal{M}, \mathcal{M}^{\prime}, \beta\right)$, where $\mathcal{M}$ and $\mathcal{M}^{\prime}$ are rank $n$ bundles on $X$ and $\beta$ is an isomorphism $\left.\left.\mathcal{M}\right|_{X-x} \stackrel{\sim}{\rightarrow} \mathcal{M}^{\prime}\right|_{X-x}$. To a dominant weight $\lambda$ of $G L_{n}\left(\overline{\mathbb{Q}}_{\ell}\right)$ we associate a closed finite-dimensional substack ${ }_{x} \overline{\mathcal{H}}_{n}^{\lambda}$ of ${ }_{x} \mathcal{H}_{n}$, which classifies the triples $\left(\mathcal{M}, \mathcal{M}^{\prime}, \beta\right)$, such that for an algebraic representation $V$ of $G L_{n}(\mathbb{k})$, whose weights are $\leq \check{\nu}$, we have the following embeddings induced by $\beta$ on the entire $X$ :

$$
V_{\mathcal{M}^{\prime}}\left(\left\langle w_{0}(\lambda), \check{\nu}\right\rangle \cdot x\right) \subset V_{\mathcal{M}} \subset V_{\mathcal{M}^{\prime}}(\langle\lambda, \check{\nu}\rangle \cdot x),
$$

where $V_{\mathcal{M}}$ is the vector bundle on $X$ associated with $V$ and the principal $G L_{n^{-}}$ bundle on $X$ corresponding to $\mathcal{M}$ (recall that $w_{0}$ stands for the permutation $\left.\left(d_{1}, d_{2}, \ldots, d_{n}\right) \mapsto\left(d_{n}, \ldots, d_{2}, d_{1}\right)\right)$.

Using this stack, we define the Hecke functor ${ }_{x} \mathrm{H}_{n}^{\lambda}: \mathrm{D}\left(\mathrm{Bun}_{n}\right) \rightarrow \mathrm{D}\left(\mathrm{Bun}_{n}\right)$ by the formula

$$
\mathcal{K} \mapsto h_{!} \rightarrow\left(h^{\leftarrow *}(\mathcal{K}) \otimes \mathrm{IC}_{\lambda}\right) \otimes \overline{\mathbb{Q}}_{\ell}\left(\frac{\operatorname{dim}\left(\mathrm{Bun}_{n}\right)}{2}\right)\left[\operatorname{dim}\left(\operatorname{Bun}_{n}\right)\right],
$$

where $h^{\leftarrow}\left(\right.$ resp., $\left.h^{\rightarrow}\right)$ sends $\left(\mathcal{M}, \mathcal{M}^{\prime}, \beta\right)$ to $\mathcal{M}$ (resp., $\left.\mathcal{M}^{\prime}\right)$, and $\mathrm{IC}_{\lambda}$ is the intersection cohomology sheaf on ${ }_{x} \overline{\mathcal{H}}_{n}^{\lambda}$.

In particular, if $\lambda$ is the $i$-th fundamental weight $\omega_{i}$, then the stack ${ }_{x} \overline{\mathcal{H}}_{n}^{\omega_{i}}$ is nothing but the preimage of $x \in X$ in $\mathcal{H}_{n}^{i}$ under supp : $\mathcal{H}_{n}^{i} \rightarrow X$. Hence ${ }_{x} \mathrm{H}_{n}^{\omega_{i}}$ is the composition of $\mathrm{H}_{n}^{i}$ followed by the restriction to $x \times \operatorname{Bun}_{n} \simeq \operatorname{Bun}_{n} \subset X \times \operatorname{Bun}_{n}$. 
The results of [Lu Gi, MV imply the following formula:

$$
{ }_{x} \mathrm{H}_{n}^{\lambda} \circ{ }_{x} \mathrm{H}_{n}^{\mu} \simeq \bigoplus_{\nu \in P_{n}^{+}}{ }_{x} \mathrm{H}_{n}^{\nu} \otimes \operatorname{Hom}_{G L_{n}}\left(V^{\nu}, V^{\lambda} \otimes V^{\mu}\right),
$$

where the notation $V^{\lambda}$ is as in Sect. 4.8,

Consider the fiber product $Z^{\lambda, x}:=\overline{\mathrm{Q}}^{0} \underset{\operatorname{Bun}_{n}^{0}}{\times}{ }_{x} \overline{\mathcal{H}}_{n}^{\lambda}$. It was proved in [FGV],

Prop. 5.3.4, that there exists a commutative diagram

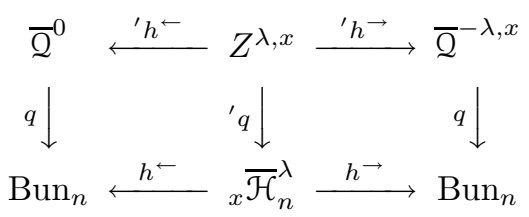

where we write $\overline{\mathrm{Q}}^{-\lambda, x}$ for $\overline{\mathrm{Q}}^{-\bar{\lambda}, \bar{x}}$ when the collection $\bar{\lambda}$ (resp., $\bar{x}$ ) consists of just one element $\lambda$ (resp., $x$ ), in the notation of Sect.4.8. According to Theorems 3 and 4 of [FGV], adapted to our present notation, we have:

$$
{ }^{\prime} h_{!} \rightarrow\left({ }^{\leftarrow * *}\left(\Psi^{0}\right) \otimes{ }^{\prime} q^{*}\left(\mathrm{IC}_{\lambda}\right)\right) \otimes \overline{\mathbb{Q}}_{\ell}\left(\frac{\operatorname{dim}\left(\operatorname{Bun}_{n}\right)}{2}\right)\left[\operatorname{dim}\left(\operatorname{Bun}_{n}\right)\right] \simeq \Psi^{-\lambda, x} .
$$

More generally, let $x^{1}, \ldots, x^{m}$ be a set of distinct points, different from $x$, and let $\bar{\mu}=\left(\mu^{0}, \mu^{1}, \ldots, \mu^{m}\right)$ be a collection of dominant weights. Set

$$
Z^{\lambda, \bar{\mu}, \bar{x}}:=\overline{\mathcal{Q}}^{-\bar{\mu}, \bar{x}} \underset{\operatorname{Bun}_{n}}{\times} \overline{\mathcal{H}}_{n}^{\lambda},
$$

where $\bar{x}=\left(x, x^{1}, \ldots, x^{m}\right)$. Denote $\bar{\lambda}=(\lambda, 0, \ldots, 0)$. We have a commutative diagram:

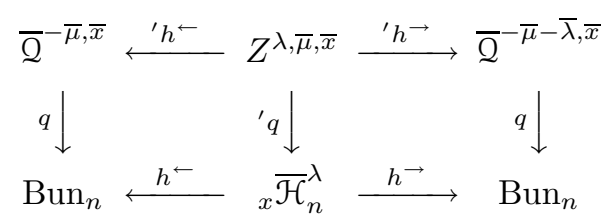

Denote by ${ }_{x}^{\prime} \mathrm{H}_{n}^{\lambda}$ the functor $\mathrm{D}\left(\overline{\mathrm{Q}}^{-\bar{\mu}, \bar{x}}\right) \rightarrow \mathrm{D}\left(\overline{\mathrm{Q}}^{-\bar{\mu}-\bar{\lambda}, \bar{x}}\right)$,

$$
\mathcal{K} \mapsto{ }^{\prime} h_{!} \rightarrow\left({ }^{\prime} h^{\leftarrow *}(\mathcal{K}) \otimes^{\prime} q^{*}\left(\mathrm{IC}_{\lambda}\right)\right) \otimes \overline{\mathbb{Q}}_{\ell}\left(\frac{\operatorname{dim}\left(\operatorname{Bun}_{n}\right)}{2}\right)\left[\operatorname{dim}\left(\operatorname{Bun}_{n}\right)\right] .
$$

Then Corollary 5.4.3 of [FGV] gives:

$$
{ }_{x}^{\prime} \mathrm{H}_{n}^{\lambda}\left(\Psi^{-\bar{\mu}, x}\right) \simeq \bigoplus_{\nu \in P_{n}^{+}} \Psi^{-\left(\nu, \mu^{1}, \ldots, \mu^{m}\right), \bar{x}} \otimes \operatorname{Hom}_{G L_{n}}\left(V^{\nu}, V^{\lambda} \otimes V^{\mu^{0}}\right) .
$$

A.2. Proof of Proposition 4.12, To simplify the notation, we consider the case when $m=1$, i.e., $\bar{\mu}=\mu$ and $\bar{x}=x$. For $m>1$ the proof is essentially the same. By construction, $Q^{\mu, x}$ and $\bar{Q}^{\mu, x}$ are substacks of $\bar{Q}^{d \cdot x} \subset \bar{Q}^{d}$.

Observe that the preimage of $d \cdot x \in X^{(d)}$ under supp : $\operatorname{Mod}_{n}^{d} \rightarrow X^{(d)}$ can be identified with the closed substack of ${ }_{x} \mathcal{H}_{n}$, which classifies those triples $\left(\mathcal{M}, \mathcal{N}^{\prime}, \beta\right)$, for which $\beta:\left.\left.\left.\mathcal{M}\right|_{X-x} \stackrel{\sim}{\rightarrow} \mathcal{M}\right|^{\prime}\right|_{X-x}$ extends to an embedding $\mathcal{M} \hookrightarrow \mathcal{M}^{\prime}$ over the entire $X$, and $\operatorname{deg}\left(\mathcal{M}^{\prime}\right)-\operatorname{deg}(\mathcal{M})=d$.

Recall the morphism $\pi: \operatorname{Mod}_{n}^{d} \rightarrow \operatorname{Coh}_{0}^{d}$. Let us denote the pull-back $\pi^{*}\left(\mathcal{L}_{E}^{d}\right) \otimes$ $\overline{\mathbb{Q}}_{\ell}\left(\frac{d \cdot n}{2}\right)[d \cdot n]$ by $\mathcal{P}_{E}^{d}$. 
It follows from the results of Lau1, Sect. 3, and [FGKV], Sect. 4.2, that the $*$-restriction of $\mathcal{P}_{E}^{d}$ to $\operatorname{supp}^{-1}(d \cdot x) \subset \operatorname{Mod}_{n}^{d}$ can be canonically identified with

$$
\bigoplus_{\lambda \in P_{n, d}^{+}} \mathrm{IC}_{-w_{0}(\lambda)} \otimes \operatorname{Hom}_{G L_{n}}\left(V^{\lambda}, \operatorname{Sym}^{d}\left(V \otimes E_{x}\right)\right) \otimes \overline{\mathbb{Q}}_{\ell}\left(\frac{d}{2}\right)[d] .
$$

Here

$$
P_{n, d}^{+}=\left\{\left(d_{1}, \ldots, d_{n}\right) \mid d_{1} \geq d_{2} \geq \ldots \geq d_{n} \geq 0, \sum_{i=1}^{n} d_{i}=d\right\}
$$

and $V=V^{(1,0, \ldots, 0)}$ stands for the defining representation of $G L_{n}$. Further, we have:

$$
\operatorname{Sym}^{d}\left(V \otimes E_{x}\right) \simeq \bigoplus_{\lambda \in P_{n, d}^{+}} V^{\lambda} \otimes E_{x}^{\lambda} .
$$

By comparing the definition of $\mathcal{W}_{E}^{d}$ with formulas (A.2), A.4 and (A.6), we obtain that the restriction of $\mathcal{W}_{E}^{d}$ to $\overline{\mathcal{Q}}^{d \cdot x}$ can be identified with

$$
\bigoplus_{\lambda \in P_{n, d}^{+}} \Psi^{w_{0}(\lambda), x} \otimes E_{w_{0}(\lambda), x} \otimes \overline{\mathbb{Q}}_{\ell}\left(\frac{d}{2}\right)[d],
$$

which is what we had to prove.

A.3. Proof of Proposition 7.5: Local computation. Consider the morphism $\tau: \overline{\mathcal{Q}}_{++}^{d} \rightarrow X \times X^{(d+1)}$, sending $\left(x, \mathcal{M},\left(s_{i}\right)\right)$ to $(x, D)$, where $D$ is the divisor of zeroes of the map $s_{n}: \Omega^{n(n-1) / 2} \rightarrow(\operatorname{det} \mathcal{M})(x)$. We start by describing explicitly the restriction of the complex $\mathcal{W}_{E,+}^{d}$ to $\overline{\mathcal{Q}}_{+}^{d} \cap \tau^{-1}(x \times D)$.

Let us write $D=d^{0} \cdot x+d^{1} \cdot x^{1}+\ldots+d^{m} \cdot x^{m}$, where $x^{i}$ are pairwise distinct and different from $x$, and $d^{0}+d^{1}+\ldots+d^{m}=d+1$.

It follows from the definitions given in Sect. 4.8 that the stack $\bar{Q}_{++}^{d} \cap \tau^{-1}(x \times D)$ is identified with $\overline{\mathcal{Q}}^{\prime \prime}, \bar{x}$, where $\bar{x}=\left(x, x^{1}, \ldots, x^{m}\right)$ and $\bar{\nu}^{\prime \prime}=\left(\nu^{0 \prime \prime}, \nu^{1}, \ldots, \nu^{m}\right)$, with $\nu^{0 \prime \prime}=\left(-1,0, \ldots, 0, d^{0}\right)$ and $\nu^{j}=\left(0, \ldots, 0, d^{j}\right), j=1, \ldots, m$. Furthermore, $\overline{\mathbf{Q}}_{+}^{d} \cap$ $\tau^{-1}(x \times D)$ is identified with the substack $\overline{\mathcal{Q}}^{\bar{\nu}^{\prime}, \bar{x}}$ of $\overline{\mathrm{Q}}^{\bar{\nu}, \bar{x}}$, where $\bar{\nu}^{\prime}=\left(\nu^{0 \prime}, \nu^{1}, \ldots, \nu^{m}\right)$, with $\nu^{0 \prime}=\left(0,-1,0, \ldots, 0, d^{0}\right)$ and $\nu^{j}, j=1, \ldots, m$, as above.

We also have a morphism $\tau: \overline{\mathcal{Q}}^{d+1} \rightarrow X^{(d+1)}$, and we identify $\tau^{-1}(D)$ with $\overline{\mathcal{Q}}^{\bar{\nu}, \bar{x}}$, where $\bar{\nu}=\left(\nu^{0}, \nu^{1}, \ldots, \nu^{m}\right)$, with $\nu^{0}=\left(0, \ldots, 0, d^{0}\right)$ and $\nu^{j}, j=1, \ldots, m$, as above.

We have a commutative diagram:

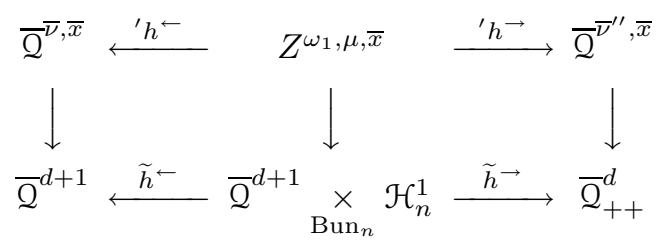

Moreover, it follows from the definitions that both squares of this diagram are Cartesian. Therefore we obtain the following formula for the restriction of the sheaf $\mathcal{W}_{E,+}^{d}=\left.\widetilde{h}_{!} \rightarrow \widetilde{h}^{\leftarrow *}\left(\mathcal{W}_{E}^{d+1}\right)\right|_{\overline{\mathcal{Q}}_{+}^{d}} \otimes \overline{\mathbb{Q}}_{\ell}\left(\frac{n-2}{2}\right)[n-2]$ to $\overline{\mathcal{Q}}_{+}^{d} \cap \tau^{-1}(x \times D)=\overline{\mathcal{Q}}^{\prime} \bar{\nu}^{\prime}$ :

$$
\left.\left.\mathcal{W}_{E,+}^{d}\right|_{\overline{\mathrm{Q}}^{\nu^{\prime}, \bar{x}}} \simeq{ }_{x}^{\prime} \mathrm{H}_{n}^{\omega_{1}}\left(\left.\mathcal{W}_{E}^{d+1}\right|_{\overline{\mathbf{Q}}^{\bar{\nu}, \bar{x}}}\right)\right|_{\overline{\mathbf{Q}}^{\bar{\nu}^{\prime}, \bar{x}}} .
$$


By Proposition 4.12

$$
\left.\mathcal{W}_{E}^{d+1}\right|_{\bar{Q}^{\nu, x}} \simeq \bigoplus_{\bar{\mu}} \Psi^{\bar{\mu}, \bar{x}} \otimes E_{\mu^{0}, x} \otimes E_{\mu^{1}, x^{1}} \otimes \ldots \otimes E_{\mu^{m}, x^{m}}
$$

where the summation is over all $\bar{\mu}=\left(\mu^{0}, \ldots, \mu^{m}\right)$ with $\mu^{j} \in w_{0}\left(P_{n, d^{j}}^{+}\right), j=$ $0, \ldots, m$.

Applying formula (A.3), we obtain

$$
\begin{array}{r}
{ }_{x}^{\prime} \mathrm{H}_{n}^{\omega_{1}}\left(\mathcal{W}_{E}^{d+1} \mid \bar{\Omega}_{\bar{Q}}^{\bar{v}, x}\right) \simeq \bigoplus_{\bar{\mu}^{\prime}} \Psi^{\bar{\mu}^{\prime}, \bar{x}} \otimes \operatorname{Hom}_{G L_{n}}\left(V_{\mu^{0}}, \operatorname{Sym}^{d^{0}}\left(V \otimes E_{x}\right) \otimes V^{*}\right) \\
\otimes E_{\mu^{1}, x^{1}} \otimes \ldots \otimes E_{\mu^{m}, x^{m}} \otimes \overline{\mathbb{Q}}_{\ell}\left(\frac{d}{2}\right)[d],
\end{array}
$$

where $\bar{\mu}^{\prime}=\left(\mu^{0 \prime}, \mu^{1}, \ldots, \mu^{m}\right)$ with $\mu^{0 \prime}$ running over the set $w_{0}\left(P_{n}^{+}\right)$, and $\mu^{j}$ running over the set $w_{0}\left(P_{n, d^{j}}^{+}\right)$for $j=1, \ldots, m$. Here $V^{*}$ is the representation of $G L_{n}\left(\overline{\mathbb{Q}}_{\ell}\right)$ dual to $V$.

We have a stratification of the stack $\bar{Q}^{\bar{\nu}^{\prime \prime}, \bar{x}}$ analogous to the stratification described in Lemma 4.10 We list only the strata that can possibly support a sheaf of the form $\Psi^{\bar{\mu}^{\prime}, \bar{x}}$. Those are $\mathcal{Q}^{\bar{\mu}^{\prime}, \bar{x}}$, where $\bar{\mu}^{\prime}=\left(\mu^{0 \prime}, \ldots, \mu^{m}\right)$ are such that $\mu^{0 \prime} \geq \nu^{0 \prime \prime}, \mu^{j} \geq \nu^{j}, j=1, \ldots, m$. (We recall that the inequality $\lambda \geq \lambda^{\prime}$ means that $\lambda$ belongs to the set $\lambda^{\prime}+R_{+}$, where $R_{+}$is the set of all linear combinations of simple roots $\alpha_{i}, i=1, \ldots, n-1$, of $G L_{n}$ with non-negative integer coefficients.)

The stratum $Q^{\bar{\mu}^{\prime}, \bar{x}}$ belongs to the substack $\bar{Q}^{\bar{\nu}^{\prime}, \bar{x}}$ if and only if in addition $\mu^{0 \prime} \geq$ $\nu^{0 \prime}=\nu^{0 \prime \prime}+\alpha_{1}$.

Recall that each sheaf $\Psi^{\bar{\mu}^{\prime}, \bar{x}}$ is the extension by zero of its restriction to the stratum $Q^{\bar{\mu}^{\prime}, \bar{x}}$. The stratum with $\bar{\mu}^{\prime}$ appearing in the summation of the RHS of formula A.10) belongs to $\bar{Q}^{\bar{\nu}^{\prime}, \bar{x}}$ if and only if $d^{0}>1, w_{0}\left(\mu^{0 \prime}\right) \in P_{n, d^{0}-1}^{+}$, and $w_{0}\left(\mu^{j}\right) \in P_{n, d^{j}}^{+}$for all $j=1, \ldots, m$. All of these strata belong to $X \times \overline{\mathrm{Q}}^{d} \subset \overline{\mathrm{Q}}_{+}^{d}$. Therefore $\mathcal{W}_{E,+}^{d}$ is supported on $X \times \overline{\mathbf{Q}}^{d} \subset \overline{\mathrm{Q}}_{+}^{d}$. This proves the first assertion of Proposition 7.5,

Furthermore, we have for $d^{0}>1$ and any $\mu^{0} \in w_{0}\left(P_{n, d^{0}-1}^{+}\right)$:

$$
\operatorname{Hom}_{G L_{n}}\left(V_{\mu^{0}}, \operatorname{Sym}^{d^{0}}\left(V \otimes E_{x}\right) \otimes V^{*}\right) \simeq E_{x} \otimes E_{\mu^{0 \prime}, x} .
$$

Combining this with formulas A.8, A.10) and A.9, we obtain the desired isomorphism $\left.\mathcal{W}_{E,+}^{d}\right|_{X \times \bar{\Omega}^{d}} \simeq E \otimes \mathcal{W}_{E}^{d}$ over the preimage of each $x \times D \subset X \times X^{(d+1)}$ in $\overline{\mathrm{Q}}_{+}^{d}$.

A.4. Proof of Proposition 7.5; Global computation. To complete the proof of Proposition [7.5 we need to show that the isomorphism $\left.\mathcal{W}_{E,+}^{d}\right|_{X \times \bar{\Omega}^{d}} \simeq E \otimes \mathcal{W}_{E}^{d}$ holds globally, and not only on each fiber $\tau^{-1}(x \times D)$.

In order to show that, we introduce one more stack, $\overline{\mathcal{Q}}_{+-}^{d}$. This is a closed substack of $\bar{Q}_{++}^{d}$ which is the preimage of the incidence divisor $X \times X^{(d)} \subset X \times$ $X^{(d+1)}$ under the morphism $\tau: \overline{\mathrm{Q}}_{++}^{d} \rightarrow X \times X^{(d+1)}$. Equivalently, the stack $\overline{\mathrm{Q}}_{+-}^{d}$ may be defined by the condition that the image of $s_{n}$ is contained in $\operatorname{det} \mathcal{M} \subset$ $(\operatorname{det} \mathcal{N})(x)$.

Let us consider the stack $\operatorname{Mod}_{n,++}^{d}$ which classifies the data $\left(x, \mathcal{M}_{0}, \mathcal{M}, \mathcal{M}_{0} \hookrightarrow\right.$ $\mathcal{M}(x))$, where $x \in X, \operatorname{deg}(\mathcal{M})-\operatorname{deg}\left(\mathcal{M}_{0}\right)=d$, and the map $\mathcal{M}_{0} \rightarrow \mathcal{M}(x)$ is such 
that the image of $\operatorname{det} \mathcal{M}_{0}$ is contained in $(\operatorname{det} \mathcal{N})(x)$ (and not just in $(\operatorname{det} \mathcal{M})(n \cdot x)$ ). Let $\operatorname{Mod}_{n,+-}^{d}$ be the closed substack of $\operatorname{Mod}_{n,++}^{d}$, where the image of $\operatorname{det} \mathcal{M}_{0}$ is contained in $\operatorname{det} \mathcal{M}$.

Consider the Cartesian product $\operatorname{Mod}_{n}^{d+1} \times \mathcal{H}_{n}^{1}$, which classifies the data

$$
\left(x, \mathcal{M}_{0}, \mathcal{M}, \mathcal{M}^{\prime}, \mathcal{M}_{0} \hookrightarrow \mathcal{M}^{\prime}, \mathcal{M} \hookrightarrow \mathcal{M}^{\prime}\right)
$$

where $\operatorname{deg}\left(\mathcal{M}^{\prime}\right)-\operatorname{deg}\left(\mathcal{M}_{0}\right)=d+1$ and $\mathcal{M}^{\prime} / \mathcal{M}$ is the simple skyscraper sheaf supported at $x$. We have a natural proper morphism

$$
\mathbf{c}: \operatorname{Mod}_{n}^{d+1} \underset{\operatorname{Bun}_{n}}{\times} \mathcal{H}_{n}^{1} \rightarrow \operatorname{Mod}_{n,++}^{d},
$$

which corresponds to "forgetting" $\mathcal{N}^{\prime}$, and a natural projection $\mathbf{b}: \operatorname{Mod}_{n}^{d+1} \underset{\operatorname{Bun}_{n}}{\times} \mathcal{H}_{n}^{1}$ $\rightarrow \operatorname{Mod}_{n}^{d+1}$, which corresponds to "forgetting" $\mathcal{M}$. We define the complex $\mathcal{P}_{E,++}^{d+1}$ on $\operatorname{Mod}_{n,++}^{d}$ as

$$
\mathcal{P}_{E,++}^{d+1}:=\mathbf{c}_{!}\left(\mathbf{b}^{*}\left(\mathcal{P}_{E}^{d+1}\right)\right) \otimes \overline{\mathbb{Q}}_{\ell}\left(\frac{n}{2}\right)[n]
$$

(recall that $\left.\mathcal{P}_{E}^{d}:=\pi^{*}\left(\mathcal{L}_{E}^{d}\right) \otimes \overline{\mathbb{Q}}_{\ell}\left(\frac{d \cdot n}{2}\right)[d \cdot n]\right)$. Let $\mathcal{P}_{E,+-}^{d+1}$ be the restriction of $\mathcal{P}_{E,++}^{d+1}$ to $\operatorname{Mod}_{n,+-}^{d}$ tensored with $\overline{\mathbb{Q}}_{\ell}\left(\frac{-1}{2}\right)[-1]$.

Now form a commutative diagram, in which the left square is Cartesian:

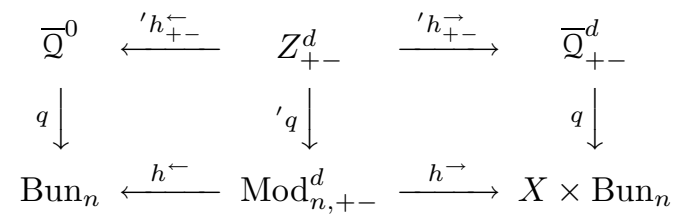

Consider the complex

$$
\mathcal{W}_{E,+-}^{d}:={ }^{\prime} h_{+-!}\left({ }^{\prime} h_{+-}^{\leftarrow}\left(\Psi^{0}\right) \otimes{ }^{\prime} q^{*}\left(\mathcal{P}_{E,+-}^{d+1}\right)\right) \otimes \overline{\mathbb{Q}}_{\ell}\left(\frac{-1}{2}\right)[-1] .
$$

Since we already know that $\mathcal{W}_{E,+}^{d}$ vanishes on $\overline{\mathbf{Q}}_{+}^{d}-\left(\overline{\mathrm{Q}}_{+}^{d} \cap \overline{\mathrm{Q}}_{+-}^{d}\right)$, it suffices to show that the restriction of $\mathcal{W}_{E,+-}^{d}$ to $\overline{\mathrm{Q}}_{+}^{d} \cap \overline{\mathrm{Q}}_{+-}^{d}$ is supported on $X \times \overline{\mathrm{Q}}^{d}$, where it is isomorphic to $E \otimes \mathcal{W}_{E}^{d}$.

Observe that $X \times \operatorname{Mod}_{n}^{d}$ is naturally a closed substack in $\operatorname{Mod}_{n,+-}^{d}$. The following result completes the proof of Proposition 7.5:

A.5. Lemma. (1) The complex $\mathcal{P}_{E,+-}^{d+1}$ is a perverse sheaf, and there is a natural surjection

$$
\mathcal{P}_{E,+-}^{d+1} \rightarrow\left(E \otimes \overline{\mathbb{Q}}_{\ell}\left(\frac{1}{2}\right)[1]\right) \otimes \mathcal{P}_{E}^{d} .
$$

(2) Let $\mathcal{K}_{E}$ be the kernel of the map (A.12). The ${ }^{*}$-restriction of the complex $' h_{+-!}\left({ }^{\prime} h_{+-}^{\leftarrow}\left(\Psi^{0}\right) \otimes{ }^{\prime} q^{*}\left(\mathcal{K}_{E}\right)\right)$ from $\overline{\mathbf{Q}}_{+-}^{d}$ to $\overline{\mathbf{Q}}_{+}^{d} \cap \overline{\mathbf{Q}}_{+-}^{d}$ vanishes identically.

A.6. An informal explanation. Before giving a formal proof of Lemma A.5, we explain the main idea behind it. In this discussion we will assume that our ground field $\mathbb{k}$ is algebraically closed.

Recall the full Hecke correspondence stack ${ }_{x} \mathcal{H}_{n}$. Observe that the fiber $(h \rightarrow)^{-1}\left(\mathcal{M}^{\prime}\right)$ of ${ }_{x} \mathcal{H}_{n}$ over $\mathcal{M}^{\prime} \in \operatorname{Bun}_{n}$ under the map $h^{\rightarrow}$ is isomorphic to the affine Grassmannian $\mathrm{Gr}_{x}$ (see, e.g., FGKV, FGV]). Let $\mathcal{O}_{x}$ be the complete local ring at 
$x$. The group $G L_{n}\left(\mathcal{O}_{x}\right)$ acts naturally on $\mathrm{Gr}_{x}$ and we say that a perverse sheaf on $\mathrm{Gr}_{x}$ is spherical if it is $G L_{n}\left(\mathcal{O}_{x}\right)$-equivariant. (In particular, every spherical perverse sheaf is smooth along the stratification of $\mathrm{Gr}_{x}$ by $\mathrm{Gr}_{x}^{\lambda}:=\mathrm{Gr}_{x} \cap_{x} \mathcal{H}_{n}^{\lambda}, \lambda \in P_{n}^{+}$.) The category of spherical perverse sheaves is known to be semi-simple and equivalent as a tensor category to the category of representations of $G L_{n}\left(\overline{\mathbb{Q}}_{\ell}\right)$, with the fiber functor being the functor of (total) global cohomology (see [Lu Gi,, $\mathrm{MV}] \mathrm{BD}]$ ).

It is possible to generalize this equivalence to the case when $x$ and $\mathcal{M}^{\prime}$ are allowed to "move" along $X \times \operatorname{Bun}_{n}$. Namely, let $\mathcal{H}_{n}$ be the stack classifying quadruples $\left(\mathcal{M}, \mathcal{M}^{\prime}, \beta, x\right)$, where $\mathcal{M}$ and $\mathcal{M}^{\prime}$ are rank $n$ bundles on $X$, and $\beta$ is an isomorphism $\left.\left.\mathcal{M}\right|_{X-x} \simeq \mathcal{M}^{\prime}\right|_{X-x}$. Then there is an equivalence between the category of perverse sheaves on $X$ equipped with $G L_{n}\left(\overline{\mathbb{Q}}_{\ell}\right)$-action and a certain subcategory of the category of perverse sheaves on $\mathcal{H}_{n}$. For each $x \in X$ this equivalence "restricts" to the equivalence of the previous paragraph.

Moreover, one can generalize this construction to the case of several points. For any partition $\mathbf{d}=\left(d^{1}, \ldots, d^{k}\right)$ of $d$, consider the open subset $\stackrel{\circ}{X}^{\mathbf{d}}$ of $X^{\left(d^{1}\right)} \times \ldots \times$ $X^{\left(d^{k}\right)}$ consisting of $k$-tuples of divisors $\left(D_{1}, \ldots, D_{k}\right)$, such that supp $D_{i} \cap \operatorname{supp} D_{j}=$ $\emptyset$, if $i \neq j$. Denote the map $\stackrel{\circ}{X}^{\mathbf{d}} \rightarrow X^{(d)}$ by $p_{\mathbf{d}}$. We introduce an abelian category $\mathcal{A}_{n}^{d}$ as follows. The objects of $\mathcal{A}_{n}^{d}$ are perverse sheaves $\mathcal{F}$ on $X^{(d)}$ equipped with a $G L_{n}\left(\overline{\mathbb{Q}}_{\ell}\right)$-action, together with the following extra structure: for each partition $\mathbf{d}$, the sheaf $p_{\mathbf{d}}^{*}(\mathcal{F})$ should carry an action of $k$ copies of $G L_{n}\left(\overline{\mathbb{Q}}_{\ell}\right)$, compatible with the original $G L_{n}\left(\overline{\mathbb{Q}}_{\ell}\right)$-action on $\mathcal{F}$ with respect to the diagonal embedding $G L_{n}\left(\overline{\mathbb{Q}}_{\ell}\right) \rightarrow$ $\left(G L_{n}\left(\overline{\mathbb{Q}}_{\ell}\right)\right)^{\times k}$. For different partitions, these actions should be compatible in the obvious sense. In addition, it is required that whenever $d^{i}=d^{j}, i \neq j$, the action of the $i$-th and $j$-th copies of $G L_{n}\left(\overline{\mathbb{Q}}_{\ell}\right)$ on $p_{\mathbf{d}}^{*}(\mathcal{F})$ commutes with the corresponding natural $\mathbb{Z}_{2}$-action on $\stackrel{\circ}{X}^{\mathbf{d}}$. The definition of morphisms in $\mathcal{A}_{n}^{d}$ is clear.

Let now $\mathcal{H}_{n}^{\mathrm{BD}, d}$ be the symmetrized version of the Beilinson-Drinfeld affine Grassmannian (see $[\mathrm{BD}]$ ). By definition, $\mathcal{H}_{n}^{\mathrm{BD}, d}$ is the ind-stack classifying quadruples $\left(\mathcal{M}, \mathcal{M} \mathcal{N}^{\prime}, D, \beta\right)$, where $\mathcal{M}, \mathcal{M}$ are as above, $D \in X^{(d)}$ and $\beta$ is an isomorphism between $\mathcal{M}$ and $\mathcal{M}^{\prime}$ away from the support of the divisor $D$. In particular, $\operatorname{Mod}_{n}^{d}$ is naturally a closed substack of $\mathcal{H}_{n}^{\mathrm{BD}, d}$, corresponding to the condition that the meromorphic map $\mathcal{M}^{\prime} \rightarrow \mathcal{M}$ defined by $\beta$ is regular.

One can introduce the notion of a spherical perverse sheaf on $\mathcal{H}_{n}^{\mathrm{BD}, d}$ and construct an equivalence between the above category $\mathcal{A}_{n}^{d}$ and the category of spherical perverse sheaves on $\mathcal{H}_{n}^{\mathrm{BD}, d}$. For example, the perverse sheaf $\operatorname{Sym}^{d}(V \otimes E) \otimes \overline{\mathbb{Q}}_{\ell}\left(\frac{d}{2}\right)[d]$, which is naturally on object of $\mathcal{A}_{n}^{d}$, goes to the sheaf $\mathcal{P}_{E}^{d}$ (considered as a sheaf on $\mathcal{H}_{n}^{\mathrm{BD}, d}$ supported on $\left.\operatorname{Mod}_{n}^{d}\right)$.

One can also define categories analogous to $\mathcal{A}_{n}^{d}$ over partially symmetrized powers of $X$. From this point of view, the sheaves on $\operatorname{Mod}_{n,+-}^{d}$ that we are interested in correspond to perverse sheaves on $X \times X^{(d)}$ equipped with $G L_{n}\left(\overline{\mathbb{Q}}_{\ell}\right)$-action and an additional structure as above.

In particular, the sheaf $\mathcal{P}_{E,+-}^{d+1}$ corresponds to the restriction to $X \times X^{(d)} \subset$ $X \times X^{(d+1)}$ of the sheaf

$$
\left(V^{*} \otimes \overline{\mathbb{Q}}_{\ell}\right) \otimes \operatorname{Sym}^{d+1}(V \otimes E) \otimes \overline{\mathbb{Q}}_{\ell}\left(\frac{d+1}{2}\right)[d+1]
$$

on $X \times X^{(d+1)}$. 
The first assertion of Lemma A.5 then translates into the statement that this restriction is perverse, and that there is a map

$$
\left.\left(V^{*} \otimes \overline{\mathbb{Q}}_{\ell}\right) \otimes \operatorname{Sym}^{d+1}(V \otimes E)\right|_{X \times X^{(d)}} \rightarrow\left(\overline{\mathbb{Q}}_{\ell} \otimes E\right) \otimes \operatorname{Sym}^{d}(V \otimes E),
$$

which becomes after a cohomological shift by $d+1$ a surjection of perverse sheaves.

The required map is induced by the obvious map

$$
\left.\overline{\mathbb{Q}}_{\ell} \otimes \operatorname{Sym}^{d+1}(V \otimes E)\right|_{X \times X^{(d)}} \rightarrow(V \otimes E) \otimes \operatorname{Sym}^{d}(V \otimes E) .
$$

(In fact, for any local system $\widetilde{E}$ on $X$, the map $\left.\operatorname{Sym}^{d+1}(\widetilde{E})\right|_{X \times X(d)} \rightarrow \widetilde{E} \otimes \operatorname{Sym}^{d}(\widetilde{E})$, which is an injection of sheaves, becomes after a cohomological shift a surjection of perverse sheaves such that $\widetilde{E} \otimes \operatorname{Sym}^{d}(\widetilde{E})[d+1]$ is the cosocle of $\left.\operatorname{Sym}^{d+1}(\widetilde{E})\right|_{X \times X^{(d)}}$ $[d+1]$; see $[\mathrm{Dr}])$.

Moreover, the kernel of the map A.13 is supported on the incidence divisor $X \times X^{(d)} \subset X \times X^{(d+1)}$, and there it satisfies the following property. Its stalk at a point $\left(x, D_{1}, \ldots, D_{k}\right)$ of $X \times X^{(d)}$ (assuming that $\operatorname{supp} D_{i} \cap \operatorname{supp} D_{j}=\emptyset, \operatorname{deg}\left(D_{i}\right)=$ $\left.d^{i}\right)$ is a $G L_{n}\left(\overline{\mathbb{Q}}_{\ell}\right)^{\times k}$-module which decomposes into irreducible components of the form $V^{\lambda^{1}} \otimes \ldots \otimes V^{\lambda^{k}}$, where at least one $\lambda^{i}$ does not belong to $P_{n, d^{i}}^{+}$. This proves the second assertion of Lemma A.5

In the proof of Lemma A.5 given below we simply perform the same manipulations as above directly in the category of sheaves on $\operatorname{Mod}_{n,+-}^{d}$.

A.7. Proof of Lemma A.5. First observe that $\mathcal{P}_{E,++}^{d+1}$ is a perverse sheaf on $\operatorname{Mod}_{n,++}^{d}$, by a standard smallness result in the theory of the affine Grassmannian. The assertion about $\mathcal{P}_{E,+-}^{d+1}$ follows because $\mathcal{P}_{E,++}^{d+1}$ has no subquotients supported over the incidence divisor $X \times X^{(d)} \rightarrow X \times X^{(d+1)}$.

To construct the surjection $\mathcal{P}_{E,+-}^{d+1} \rightarrow\left(E \otimes \overline{\mathbb{Q}}_{\ell}\left(\frac{-1}{2}\right)[-1]\right) \otimes \mathcal{P}_{E}^{d}$ we introduce the stack $^{\prime} \operatorname{Mod}_{n}^{d+1}=\operatorname{Mod}_{n}^{d+1} \underset{X^{(d+1)}}{\times}\left(X \times X^{(d)}\right)$.

We consider two perverse sheaves on it. The first one, denoted by $\mathcal{F}_{1}$, is the pullback of $\mathcal{P}_{E}^{d+1}$ under ${ }^{\prime} \operatorname{Mod}_{n}^{d+1} \rightarrow \operatorname{Mod}_{n}^{d+1}$. To construct the other sheaf, consider the morphism a : $\operatorname{Mod}_{n}^{d} \underset{\operatorname{Bun}_{n}}{\times} \mathcal{H}_{n}^{1} \rightarrow{ }^{\prime} \operatorname{Mod}_{n}^{d+1}$ defined by sending $\left(\mathcal{M}{ }_{0}, \mathcal{M}, \mathcal{M}^{\prime}, \mathcal{M}_{0} \subset\right.$ $\left.\mathcal{M}, \mathcal{M} \subset \mathcal{M}^{\prime}\right)$ to $\left(\mathcal{M}_{0} \subset \mathcal{M}^{\prime}\right) \times(x, D)$, where $D=\tau\left(\mathcal{M}_{0} \subset \mathcal{M}\right)$. The second perverse sheaf $\mathcal{F}_{2}$ is by definition

$$
\text { a! }(\mathrm{id} \times \operatorname{supp})^{*}\left(\mathcal{P}_{E}^{d} \otimes E\right) \otimes \overline{\mathbb{Q}}_{\ell}\left(\frac{n}{2}\right)[n],
$$

where (id $\times \operatorname{supp}): \operatorname{Mod}_{n}^{d} \underset{\operatorname{Bun}_{n}}{\times} \mathcal{H}_{n}^{1} \rightarrow \operatorname{Mod}_{n}^{d} \underset{\operatorname{Bun}_{n}}{\times} X$. (Thus, $\mathcal{F}_{1}$ corresponds to the sheaf $\left.\overline{\mathbb{Q}}_{\ell} \otimes \operatorname{Sym}^{d+1}(V \otimes E)\right|_{X \times X^{(d)}} \otimes \overline{\mathbb{Q}}_{\ell}\left(\frac{d+1}{2}\right)[d+1]$ and $\mathcal{F}_{2}$ corresponds to the sheaf $(V \otimes E) \otimes \operatorname{Sym}^{d}(V \otimes E) \otimes \overline{\mathbb{Q}}_{\ell}\left(\frac{d+1}{2}\right)[d+1]$ on $X \times X^{(d)}$.)

There is a natural surjective map $\mathcal{F}_{1} \rightarrow \mathcal{F}_{2}$. Now the desired map $\mathcal{P}_{E,+-}^{d+1} \rightarrow$ $\left(E \otimes \overline{\mathbb{Q}}_{\ell}\left(\frac{1}{2}\right)[1]\right) \otimes \mathcal{P}_{E}^{d}$ is obtained from the map $\mathcal{F}_{1} \rightarrow \mathcal{F}_{2}$ by adjunction. It is surjective, because $\left(E \otimes \overline{\mathbb{Q}}_{\ell}\left(\frac{1}{2}\right)[1]\right) \otimes \mathcal{P}_{E}^{d}$ has no subquotients supported on proper closed substacks. This completes the proof of part (1) of the lemma.

To prove part (2), we choose $x \times D \in X \times X^{(d)}$ and calculate the restriction of $\mathcal{K}_{E}$ to its preimage in $\operatorname{Mod}_{n,+-}^{d}$. To simplify notation, assume that $D$ is of the form $d \cdot x$. 
The preimage of $x \times d \cdot x \in X \times X^{(d)}$ under $\operatorname{Mod}_{n,+-}^{d} \stackrel{\text { supp }}{\longrightarrow} X \times X^{(d)}$ is naturally a closed substack of ${ }_{x} \mathcal{H}_{n}$. Using formula (A.4), we obtain that the restriction to $\tau^{-1}(x \times d \cdot x)$ of the surjection $\mathcal{P}_{E,+-}^{d+1} \rightarrow\left(E \otimes \overline{\mathbb{Q}}_{\ell}\left(\frac{1}{2}\right)[1]\right) \otimes \mathcal{P}_{E}^{d}$ can be identified with the map

$$
\begin{aligned}
& \bigoplus \mathrm{PC}_{-w_{0}(\lambda)} \otimes \operatorname{Hom}_{G L_{n}}\left(V^{\lambda}, \operatorname{Sym}^{d+1}\left(V \otimes E_{x}\right) \otimes V^{*}\right) \otimes \overline{\mathbb{Q}}_{\ell}\left(\frac{d+1}{2}\right)[d+1] \\
& \rightarrow \bigoplus_{\lambda \in P_{n}^{+}} \mathrm{IC}_{-w_{0}(\lambda)} \otimes \operatorname{Hom}_{G L_{n}}\left(V^{\lambda}, E_{x} \otimes \operatorname{Sym}^{d}\left(V \otimes E_{x}\right)\right) \otimes \overline{\mathbb{Q}}_{\ell}\left(\frac{d+1}{2}\right)[d+1] .
\end{aligned}
$$

The kernel of this map is nothing but the restriction of $\mathcal{K}_{E}$ to the preimage of $x \times d \cdot x \in X \times X^{(d)}$ in $\operatorname{Mod}_{n,+-}^{d}$. It is clear that if the summand corresponding to the sheaf $\mathrm{IC}_{-w_{0}(\lambda)}$ appears in $\mathcal{K}_{E}$, then $\lambda \notin P_{n, d}^{+}$. Therefore, the required vanishing follows from formula A.2 and Lemma 4.10.

\section{ACKNOWLEDGMENTS}

We express our gratitude to D. Kazhdan for his collaboration in [FGKV], which has influenced this work. We also thank V. Drinfeld, D. Kazhdan, and I. Mirković for valuable discussions.

\section{REFERENCES}

[AC] J. Arthur, L. Clozel, Simple Algebras, Base Change, and the Advanced Theory of the Trace Formula, Annals of Mathematical Studies 120, Princeton University Press, 1989. MR 90m:22041]

[BBD] A. Beilinson, J. Bernstein, P. Deligne, Faisceaux pervers, Astérisque 100 (1982). MR 86g:32015

[BD] A. Beilinson, V. Drinfeld, Quantization of Hitchin's integrable system and Hecke eigensheaves, Preprint, available at http://www.math.uchicago.edu/ benzvi.

$[\mathrm{BM}] \mathrm{W}$. Borho, R. MacPherson, Representations des groups de Weyl et homologie d'intersection pour les varits nilpotents, C.R. Acad. Sci. Paris 292 (1981) 410-431. MR 82f:14002

[BG] A. Braverman, D. Gaitsgory, Geometric Eisenstein series, Preprint math.AG/9912097.

[CS] W. Casselman, J. Shalika, The unramified principal series of p-adic groups II. The Whittaker function, Comp. Math. 41 (1980) 207-231. MR 83i:22027

[CPS] J.W. Cogdell, I.I. Piatetskii-Shapiro, Converse theorems for $G L_{n}$, Publ. IHES 79 (1994) 157-214. MR 95m:22009

[De] P. Deligne, La conjecture de Weil II, Publ. IHES 52 (1981) 313-428. MR 83c:14017

[Dr] V.G. Drinfeld, Two-dimensional $\ell$-adic representations of the fundamental group of a curve over a finite field and automorphic forms on GL(2), Amer. J. Math. 105 (1983) 85-114. MR 84i:12011

[FGKV] E. Frenkel, D. Gaitsgory, D. Kazhdan, K. Vilonen, Geometric realization of Whittaker functions and the Langlands correspondence, Journal of AMS 11 (1998) 451-484. MR 99f: 11148

[FGV] E. Frenkel, D. Gaitsgory, K. Vilonen, Whittaker patterns in the geometry of moduli spaces of bundles on curves, Annals of Math. 153 (2001) 699-748.

[Fu] W. Fulton, Young Tableaux, Cambridge University Press, 1997. MR 99f:05119

[Ga] D. Gaitsgory, Automorphic sheaves and Eisenstein series, Ph.D. Thesis, 1997.

[Gi] V. Ginzburg, Perverse sheaves on a loop group and Langlands duality, Preprint alggeom/9511007.

[Il] L. Illusie, Théorie de Brauer et Caractéristique d'Euler-Poincaré (d'après P. Deligne), Astérisque 82-93 (1981) 161-172. MR 83m:14014

[K] D. Kazhdan, On lifting, in Lie Group Representations II, Lect. Notes in Math 1041, pp. 209-249. MR 86h:22029 
[Laf] L. Lafforgue, Chtoucas de Drinfeld et correspondance de Langlands, Prépublication 2000-62, Université de Paris-Sud.

[Lau1] G. Laumon, Correspondance de Langlands géométrique pour les corps de fonctions, Duke Math. J. 54 (1987) 309-359. MR 88g:11086

[Lau2] G. Laumon, Faisceaux automorphes pour $G L_{n}$ : la première construction de Drinfeld, Preprint alg-geom/9511004 (1995).

[Lau3] G. Laumon, Transformation de Fourier généralisée, Preprint alg-geom/9603004.

[LMB] G. Laumon, L. Moret-Bailly, Champs algébriques, Ergebnisse der Mathematik und ihrer Grenzgebiete. 3. Folge (A Series of Modern Surveys in Mathematics) 39, Springer-Verlag, Berlin, 2000. MR 2001f:14006

[Lu] G. Lusztig, Singularities, character formulas, and a q-analogue of weight multiplicities, Astérisque 101 (1983) 208-229. MR 85m:17005

[Ly1] S. Lysenko, Orthogonality relations between the automorphic sheaves attached to 2dimensional irreducible local systems on a curve, Ph.D. thesis, 1999.

[Ly2] S. Lysenko, Geometric Rankin-Selberg method for $G L_{n}$, Preprint (2000).

[MV] I. Mirković, K. Vilonen, Perverse sheaves on affine Grassmannians and Langlands duality, Math. Res. Lett. 7 (2000) 13-24. MR 2001h:14020

[PS1] I.I. Piatetskii-Shapiro, Euler subgroups, in Lie Groups and Their Representations, ed. I.M. Gelfand, pp. 597-620, Adam Hilder Publ., 1975. MR 53:10720

[PS2] I.I. Piatetskii-Shapiro, Zeta-functions of $G L(n)$, Preprint of University of Maryland, 1976.

[R] M. Rothstein, Connections on the total Picard sheaf and the KP hierarchy, Acta Applicandae Mathematicae 42 (1996) 297-308. MR 97b:14037

[Sha] J.A. Shalika, The multiplicity one theorem for $G L_{n}$, Ann. Math. 100 (1974) 171-193. MR 50:545

[Shi] T. Shintani, On an explicit formula for class 1 Whittaker functions on $G L_{n}$ over $\mathfrak{P}$-adic fields, Proc. Japan Acad. 52 (1976) 180-182. MR 53:10991

[Sp] T. Springer, Quelques applications de la cohomologie d'intersection, Seminaire Bourbaki 589, Astérisque 92-93 (1982) 410-431. MR 85i:32016b

[T] J. Towber, Young symmetry, the flag manifold, and representations of $G L(n)$, J. Algebra 61 (1978) 414-462. MR 83d:15022

Department of Mathematics, University of California, Berkeley, California 94720

Department of Mathematics, Harvard University, Cambridge, Massachusetts 02138

Department of Mathematics, Northwestern University, Evanston, Illinois 60208 